\title{
Modeling and analysis of a within-host HIV/HTLV-I co-infection
}

\author{
A. M. Elaiw ${ }^{1,2}$ (D) N. H. AIShamrani ${ }^{1,3}$ (D)
}

Received: 23 July 2020 / Accepted: 7 November 2020/Published online: 29 March 2021

(C) Sociedad Matemática Mexicana 2021

\begin{abstract}
Human immunodeficiency virus (HIV) and human T-lymphotropic virus type I (HTLVI) are two retroviruses that attack the $\mathrm{CD} 4{ }^{+} \mathrm{T}$ cells and impair their functions. Both HIV and HTLV-I can be transmitted between individuals through direct contact with certain body fluids from infected individuals. Therefore, a person can be co-infected with both viruses. HIV causes acquired immunodeficiency syndrome (AIDS), while HTLV-I is the causative agent for adult T-cell leukemia (ATL) and HTLV-I-associated myelopathy/tropical spastic paraparesis (HAM/TSP). Several mathematical models have been developed in the literature to describe the within-host dynamics of HIV and HTLV-I mono-infections. However, modeling a within-host dynamics of HIV/HTLV-I co-infection has not been involved. The present paper is concerned with the formulation and investigation of a new HIV/HTLV-I co-infection model under the effect of Cytotoxic $\mathrm{T}$ lymphocytes (CTLs) immune response. The model describes the interaction between susceptible $\mathrm{CD}^{+}{ }^{+} \mathrm{T}$ cells, silent HIV-infected cells, active HIV-infected cells, silent HTLV-infected cells, Tax-expressing HTLV-infected cells, free HIV particles, HIV-specific CTLs and HTLV-specific CTLs. The HIV can spread by virus-to-cell transmission. On the other side, HTLV-I has two modes of transmission, (i) horizontal transmission via direct cell-to-cell contact through the virological synapse, and (ii) vertical transmission through the mitotic division of Tax-expressing HTLV-infected cells. The well-posedness of the model is established by showing that the solutions of the model are nonnegative and bounded. We define a set of threshold parameters which govern the existence and stability of all equilibria of the model. We explore the global asymptotic stability of all equilibria by utilizing Lyapunov function and LyapunovLaSalle asymptotic stability theorem. We have presented numerical simulations to justify the applicability and effectiveness of the theoretical results. In addition, we evaluate the effect of HTLV-I infection on the HIV dynamics and vice versa.
\end{abstract}

Keywords HIV/HTLV-I co-infection · Global stability · Mitotic transmission · CTLmediated immune response $\cdot$ Lyapunov function 
Mathematics Subject Classification 34D20 $\cdot 34$ D23 $\cdot$ 37N25 $\cdot 92$ B05

\section{Introduction}

Nowadays, humans are vulnerable to infection with many different viruses such as human immunodeficiency virus (HIV), human T-lymphotropic virus type I (HTLV$\mathrm{I})$, hepatitis $\mathrm{B}$ virus (HBV), hepatitis $\mathrm{C}$ virus (HCV), dengue virus and lastly coronavirus. These viruses cause many fatal diseases. HIV is a retrovirus that infects the susceptible $\mathrm{CD} 4^{+} \mathrm{T}$ cell and destroys its functions. Acquired immunodeficiency syndrome (AIDS) is the advanced stage of HIV infection. An individual can be infected with HIV by direct contact with certain body fluids (blood, semen (cum), pre-seminal fluid (pre-cum), vaginal fluids, rectal fluids and breast milk) from an HIV-infected individual. Till now, the available antiviral treatments can significantly suppress HIV replication but they can not eliminate the HIV from the body. According to global health observatory (GHO 2018) data of HIV/AIDS published by WHO [1] that says, globally, about 37.9 million HIV-infected people in 2018, 1.7 million newly HIV-infected and 770,000 HIV-related death in the same year. During the last decades, mathematical modeling of within-host HIV infection has witnessed a significant development. Nowak and Bangham [2] have introduced an initial HIV infection model to describe the interaction between three compartments, susceptible $\mathrm{CD}^{+}{ }^{+} \mathrm{T}$ cells $(S)$, active HIV-infected cells $(I)$ and free HIV particles $(V)$. Silent viral reservoirs remain one of the major hurdles for eradicating the HIV by current antiviral therapy [3]. Silent HIV-infected cells include HIV virions but do not produce them until they become activated. Mathematical modeling of HIV dynamics with silent infected cells can help in predicting the effect of antiviral drug efficacy on HIV progression [4]. Rong and Perelson [5] have included the silent infected cells in the initial HIV model presented in [2] as:

$$
\left\{\begin{array}{l}
\dot{S}=\rho-\alpha S-\eta_{1} S V \\
\dot{L}=(1-\beta) \eta_{1} S V-(\lambda+\gamma) L \\
\dot{I}=\beta \eta_{1} S V+\lambda L-a I \\
\dot{V}=b I-\varepsilon V
\end{array}\right.
$$

where $S=S(t), L=L(t), I=I(t)$ and $V=V(t)$ are the concentrations of susceptible CD $4^{+} \mathrm{T}$ cells, silent HIV-infected cells, active HIV-infected cells and free HIV particles at time $t$, respectively. The susceptible $\mathrm{CD}^{+} \mathrm{T}$ cells are produced at specific constant rate $\rho$. The HIV virions can replicate using virus-to-cell transmission. The term $\eta_{1} S V$ refers to the rate at which new infectious appears by virusto-cell contact between free HIV particles and susceptible $\mathrm{CD}^{+}{ }^{+} \mathrm{T}$ cells. Silent HIVinfected cells are transmitted to be active at rate $\lambda L$. The free HIV particles are generated at rate $b I$. The natural death rates of the susceptible $\mathrm{CD} 4^{+} \mathrm{T}$ cells, silent $\mathrm{HIV}$-infected cells, active HIV-infected cells and free HIV particles are given by $\alpha S$, $\gamma L, a I$ and $\varepsilon V$, respectively. A fraction $\beta \in(0,1)$ of new HIV-infected cells will be active, and the remaining part $1-\beta$ will be silent. Over past decades, mathematical modeling and analysis of HIV mono-infection with both silent and active HIV- 
infected cells have witnessed a significant development (see e.g., [6-13] and the review article [3]).

HTLV-I can lead to two diseases, adult T-cell leukemia (ATL) and HTLV-Iassociated myelopathy/tropical spastic paraparesis (HAM/TSP). HTLV-1 can be transmitted to human from sexual contact, needle sharing, contaminated blood products and breastfeeding [14]. HTLV-I is a global epidemic that infects about 1025 million persons [15]. The infection is endemic in the Caribbean, southern Japan, the Middle East, South America, parts of Africa, Melanesia and Papua New Guinea [16]. HTLV-I is a provirus that targets the susceptible CD4 ${ }^{+} \mathrm{T}$ cells. HTLV-I is a single-stranded RNA retrovirus that reverse transcribe its RNA genome into a proviral DNA copy which in turn reaches the host chromatin and integrates into the DNA of the host genome, at which point the virus is referred to as a provirus. Later, cell infected with this virus enters a silent period and it is not capable to produce DNA and infects susceptible cells. Although, silent HTLV-infected cells can survive for a long-lasting time, however, they may be suddenly activated by antigen and become able to infect susceptible cells. During the primary infection stage of HTLV-I, the proviral load can reach high level, approximately 30-50\% [17]. Unlike in the case of HIV infection, however, only a small percentage of infected individuals develop the disease and 2-5\% percent of HTLV-I carriers develop symptoms of ATL and another 0.25-3\% develop HAM/TSP [18]. Many researchers have been concerned to study mathematical modeling and analysis of HTLV-I mono-infection in several works [19-21].

There are some differences between HIV and HTLV-I. HIV can break free from a CD $4^{+} \mathrm{T}$ cell and infect other susceptible $\mathrm{CD} 4^{+} \mathrm{T}$ cell, while cell-free HTLV-I does not trigger infection. HTLV-I has two modes of transmission, the first is the horizontal transmission via direct cell-to-cell contact through the virological synapse [22], and the second is the vertical transmission through the mitotic division of Tax-expressing HTLV-infected cells [23]. Tax-expressing HTLV-infected cells proliferate faster than susceptible $\mathrm{CD} 4^{+} \mathrm{T}$ cells and silent HTLV-infected cells. This leads to an increase of proviral load. Therefore, vertical mitotic transmission plays an important role in the persistence of HTLV-I infection [23]. Li and Lim [24] have formulated an HTLV-I dynamics model that takes into account both horizontal and vertical routs of transmission as:

$$
\left\{\begin{array}{l}
\dot{S}=\rho-\alpha S-\eta_{2} S Y \\
\dot{E}=\varphi \eta_{2} S Y+\kappa r^{*} Y\left(1-\frac{S+E}{K}\right)-(\psi+\omega) E, \\
\dot{Y}=\psi E-\delta^{*} Y
\end{array}\right.
$$

where $S=S(t), E=E(t)$ and $Y=Y(t)$ are the concentrations of susceptible $\mathrm{CD} 4^{+} \mathrm{T}$ cells, silent HTLV-infected cells and Tax-expressing HTLV-infected cells, at time $t$, respectively. The rate at which new infectious appears by cell-to-cell contact between Tax-expressing HTLV-infected cells and susceptible CD4 ${ }^{+} \mathrm{T}$ cells is assumed to be $\eta_{2} S Y$. The fraction $\varphi \in(0,1)$ is the probability of new HTLV infections via horizontal transmission could enter a silent period. The other route of transmission for HTLV is the vertical caused by selective expansion of Tax- 
expressing $\mathrm{CD}^{+} \mathrm{T}$ cells that are driven into proliferation by HTLV Tax gene at a rate $r^{*} Y\left(1-\frac{S+E}{K}\right)$, where $K$ is the $\mathrm{CD}^{+} \mathrm{T}$ cells carrying capacity. The term $\kappa r^{*} Y\left(1-\frac{S+E}{K}\right)$ accounts for the HTLV-infected cells that being silent and, therefore, escaping from the immune system, where $\kappa \in(0,1)$. The natural death rates of the silent HTLV-infected cells and Tax-expressing HTLV-infected cells are represented by $\omega E$ and $\delta^{*} Y$, respectively. The term $\psi E$ accounts for the rate of silent HTLVinfected cells that become Tax-expressing HTLV-infected cells. Asquith and Bangham [25] have been reported that, even in the presence of rapid selective mitotic division, target cell populations are less than the total $\mathrm{CD} 4^{+} \mathrm{T}$ cells carrying capacity i.e. $S+E<K$. Therefore, Lim and Maini [15] have replaced the logistic term $r^{*} Y\left(1-\frac{S+E}{K}\right)$ by an exponential growth term $r^{*} Y$.

Cytotoxic T lymphocytes (CTLs) are recognized as the significant component of the human immune response against viral infections. CTLs inhibit viral replication and kill the cells which are infected by viruses. In fact, CTLs and antibodies are necessary and universal to control HIV infection for years [26]. The incorporation of the immune response in the HIV dynamics models gives us a better understanding of within-host HIV dynamics. During recent years, great efforts have been made to formulate and analyze the within-host HIV mono-infection models under the influence of CTL immune response (see e.g. [2] and [27]). In [28, 29], silent HIVinfected cells have been included in the HIV dynamics models with CTL immune response. In the case of HTLV-I infection, it has been reported in [25] and [30] that the CTLs play an effective role in controlling such infection. CTLs can recognize and kill the Tax-expressing HTLV-infected cells, moreover, they can reduce the proviral load. In the literature, several mathematical models have been proposed to describe the dynamics of HTLV-I under the effect of CTL immune response (see e.g. [16] and [31-34]). HTLV-I dynamics models with the mitotic division of Taxexpressing HTLV-infected cells and CTL immune response have been developed in [15, 35-37]. Li and Zhou [36] have assumed that Tax-expressing HTLV-infected cells proliferate at rate $r^{*} Y$, with $(1-\kappa) r^{*} Y$ staying in the Tax-expressing HTLVinfected cells compartment, while $\kappa r^{*} Y$ being silent and, therefore, escaping from the immune system.

Simultaneous infection by HIV and HTLV-I and the etiology of their pathogenic and disease outcomes have become a global health matter over the past 10 years. The importance of studying HIV/HTLV-I co-infection comes from the fact that both viruses share the same ways of transmission in a population as mentioned above. This means that co-infection with both viruses can occurred in the areas where both viruses are endemic [38]. Although $\mathrm{CD}^{+} \mathrm{T}$ cells are the major targets of both HIV and HTLV-I, however, these viruses present a different biological behavior that causes diverse impacts on host immunity and ultimately leads to numerous clinical diseases [39]. It has been reported that the HTLV-I co-infection rate among HIV infected patients as increase as 100 to 500 times in comparison with the general population [40]. In seroepidemiologic studies, it has been recorded that HIVinfected patients are more exposure to be co-infected with HTLV-I, and vice versa compared to the general population [41]. HIV/HTLV-I co-infection is usually found in individuals of specific ethnic or who belonged to geographic origins where these 
viruses are simultaneously endemic [42]. As an example, the co-infection rates in individuals living in Bahia have reached $16 \%$ of HIV-infected patients [43]. The prevalence of dual infection with HIV and HTLV-I has become more widely in several geographical regions throughout the world such as South America, Europe, the Caribbean, Bahia (Brazil), Mozambique (Africa), and Japan [41, 43]. HIV and HTLV-I dual infection appears to have an overlap on the course of associated clinical outcomes with both viruses [41]. Several reports have concluded that HIV/ HTLV-I co-infected patients were found to have an increase of CD4 ${ }^{+} \mathrm{T}$ cells count in comparison with HIV mono-infected patients, although there is no evident to result in a better immune response $[39,44]$. Indeed, simultaneously infected patients by both viruses with $\mathrm{CD} 4^{+} \mathrm{T}$ counts greater than 200 cells $/ \mathrm{mm}^{3}$ are more exposure to have other opportunistic infections as compared with HIV mono-infected patients who have similar $\mathrm{CD}^{+} \mathrm{T}$ counts [44]. Studies have reported that higher mortality and shortened survival rates were accompany with co-infected individuals more than mono-infected individuals [45]. Considering the natural history of HIV, many researchers have noted that co-infection with HIV and HTLV-I can accelerate the clinical progression to AIDS. On the other hand, HIV can adjust HTLV-I expression in co-infected individuals which leads them to a higher risk of developing HTLV-I related diseases such as ATL and TSP/HAM [40, 41, 45].

Although a great number of mathematical HIV and HTLV-I mono-infections models has been developed and analyzed, however, to the best of our knowledge there is no mathematical model for HIV/HTLV-I co-infection. Therefore, our aim in the present paper is to formulate a new HIV/HTLV-I co-infection model. The HIV can spread via virus-to-cell transmission, while HTLV-I has two routes of transmission, (i) horizontal transmission via direct cell-to-cell contact through the virological synapse, and (ii) vertical transmission through the mitotic division of Tax-expressing HTLV-infected cells. We first show that the model is well-posed by establishing that the solutions of the model are nonnegative and bounded. We calculate all equilibria and derive a set of threshold parameters which govern the existence and stability of the equilibria of the model. We study the global stability of equilibria by constructing suitable Lyapunov functions and utilizing LyapunovLaSalle asymptotic stability theorem. We conduct some numerical simulations to illustrate the theoretical results.

We remark that, our proposed HIV/HTLV-I co-infection model can be developed and extended to incorporate different biological phenomena such as intracellular time delay [46-50], reaction-diffusion [51, 52] and stochastic interactions [53].

\section{Model formulation}

We set up an ordinary differential equation (ODE) model that describes the change of concentrations of eight compartments with respect to time $t$; susceptible (uninfected) CD $4^{+}$T cells $S(t)$, silent HIV-infected cells $L(t)$, active HIV-infected cells $I(t)$, silent HTLV-infected cells $E(t)$, Tax-expressing HTLV-infected cells $Y(t)$, free HIV particles $V(t)$, HIV-specific CTLs $C^{I}(t)$ and HTLV-specific CTLs $C^{Y}(t)$. The dynamics of HIV/ HTLV-I co-infection is schematically shown in the transfer diagram given in Fig. 1. 
Our proposed model is given by the following system of ODEs:

$$
\left\{\begin{array}{l}
\dot{S}=\rho-\alpha S-\eta_{1} S V-\eta_{2} S Y, \\
\dot{L}=(1-\beta) \eta_{1} S V-(\lambda+\gamma) L, \\
\dot{I}=\beta \eta_{1} S V+\lambda L-a I-\mu_{1} C^{I} I, \\
\dot{E}=\varphi \eta_{2} S Y+\kappa r^{*} Y-(\psi+\omega) E \\
\dot{Y}=\psi E+(1-\kappa) r^{*} Y-\delta^{*} Y-\mu_{2} C^{Y} Y, \\
\dot{V}=b I-\varepsilon V \\
\dot{C}^{I}=\sigma_{1} C^{I} I-\pi_{1} C^{I} \\
\dot{C}^{Y}=\sigma_{2} C^{Y} Y-\pi_{2} C^{Y}
\end{array}\right.
$$

where $\left(S, L, I, E, Y, V, C^{I}, C^{Y}\right)=\left(S(t), L(t), I(t), E(t), Y(t), V(t), C^{I}(t), C^{Y}(t)\right)$. The term $\mu_{1} C^{I} I$ is the killing rate of active HIV-infected cells due to their specific immunity. The term $\mu_{2} C^{Y} Y$ is the killing rate of Tax-expressing HTLV-infected cells due to their specific immunity. The proliferation and death rates for both effective HIV-specific CTLs and HTLV-specific CTLs are given by $\sigma_{1} C^{I} I, \sigma_{2} C^{Y} Y$, $\pi_{1} C^{I}$ and $\pi_{2} C^{Y}$, respectively. All remaining parameters have the same biological meaning as explained in the previous section. Table 1 summarizes all parameters and their definitions.

In [15], it is assumed that $r^{*}<v^{*}=\min \left\{\alpha, \omega, \delta^{*}\right\}$, which corresponds to experimental evidence indicating that the proliferation rate of HTLV-infected cells is generally lower than the rate of removal due to natural death. Since $r^{*}<\delta^{*}$ and $0<\kappa<1$, then $(1-\kappa) r^{*}<\delta^{*}$ and

$$
\delta^{*}-(1-\kappa) r^{*}>0 .
$$

Let $\delta=\delta^{*}-(1-\kappa) r^{*}$ and $r=\kappa r^{*}$. Then, system (3) will take the following form of ODEs:

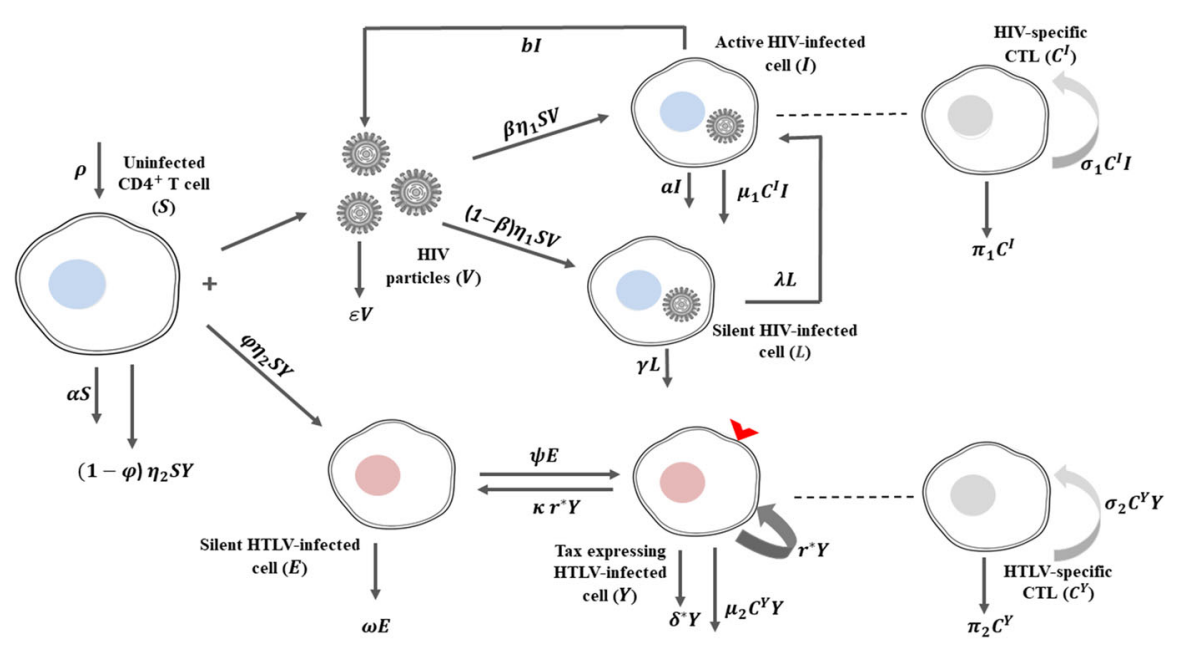

Fig. 1 The schematic diagram of the HIV/HTLV-I co-infection dynamics in vivo 
Table 1 Parameters of model (3) and their interpretations

\begin{tabular}{|c|c|}
\hline Parameter & Description \\
\hline$\rho$ & Recruitment rate for the susceptible $\mathrm{CD} 4^{+} \mathrm{T}$ cells \\
\hline$\alpha$ & Natural mortality rate constant for the susceptible $\mathrm{CD} 4^{+} \mathrm{T}$ cells \\
\hline$\eta_{1}$ & Virus-cell incidence rate constant between free HIV particles and susceptible $\mathrm{CD} 4^{+} \mathrm{T}$ cells \\
\hline$\eta_{2}$ & $\begin{array}{l}\text { Cell-cell incidence rate constant between Tax-expressing HTLV-infected cells and } \\
\text { susceptible CD } 4^{+} \mathrm{T} \text { cells }\end{array}$ \\
\hline$\beta \in(0,1)$ & $\begin{array}{l}\text { Fraction coefficient accounts for the probability of new HIV-infected cells could be active, } \\
\text { and the remaining part } 1-\beta \text { will be silent }\end{array}$ \\
\hline$\gamma$ & Death rate constant of silent HIV-infected cells \\
\hline$a$ & Death rate constant of active HIV-infected cells \\
\hline$\mu_{1}$ & Killing rate constant of active HIV-infected cells due to HIV-specific CTLs \\
\hline$\mu_{2}$ & Killing rate constant of Tax-expressing HTLV-infected cells due to HTLV-specific CTLs \\
\hline$\varphi \in(0,1)$ & $\begin{array}{l}\text { Probability of new HTLV infections via horizontal transmission could be enter a silent } \\
\text { period }\end{array}$ \\
\hline$\lambda$ & Transmission rate constant of silent HIV-infected cells that become active HIV-infected cells \\
\hline$\psi$ & $\begin{array}{l}\text { Transmission rate constant of silent HTLV-infected cells that become Tax-expressing } \\
\text { HTLV-infected cells }\end{array}$ \\
\hline$\omega$ & Death rate constant of silent HTLV-infected cells \\
\hline$\delta^{*}$ & Death rate constant of Tax-expressing HTLV-infected cells \\
\hline$\kappa \in(0,1)$ & Probability of new HTLV infections via mitosis could be enter a silent period \\
\hline$r^{*}$ & Proliferation rate constant for newly HTLV-infected cells from mitosis \\
\hline$b$ & Generation rate constant of new HIV particles \\
\hline$\varepsilon$ & Death rate constant of free HIV particles \\
\hline$\sigma_{1}$ & Proliferation rate constant of HIV-specific CTLs \\
\hline$\sigma_{2}$ & Proliferation rate constant of HTLV-specific CTLs \\
\hline$\pi_{1}$ & Decay rate constant of HIV-specific CTLs \\
\hline$\pi_{2}$ & Decay rate constant of HTLV-specific CTLs \\
\hline
\end{tabular}

$$
\left\{\begin{array}{l}
\dot{S}=\rho-\alpha S-\eta_{1} S V-\eta_{2} S Y, \\
\dot{L}=(1-\beta) \eta_{1} S V-(\lambda+\gamma) L, \\
\dot{I}=\beta \eta_{1} S V+\lambda L-a I-\mu_{1} C^{I} I, \\
\dot{E}=\varphi \eta_{2} S Y+r Y-(\psi+\omega) E, \\
\dot{Y}=\psi E-\delta Y-\mu_{2} C^{Y} Y, \\
\dot{V}=b I-\varepsilon V \\
\dot{C}^{I}=\sigma_{1} C^{I} I-\pi_{1} C^{I} \\
\dot{C}^{Y}=\sigma_{2} C^{Y} Y-\pi_{2} C^{Y} .
\end{array}\right.
$$

\section{Preliminaries}

Let $\Omega_{j}>0, j=1, \ldots, 5$ and define 


$$
\begin{aligned}
\Theta=\{ & \left(S, L, I, E, Y, V, C^{I}, C^{Y}\right) \in \mathbb{R}_{\geq 0}^{8}: 0 \leq S(t), L(t), I(t) \leq \Omega_{1} \\
& \left.0 \leq E(t), Y(t) \leq \Omega_{2}, 0 \leq V(t) \leq \Omega_{3}, 0 \leq C^{I}(t) \leq \Omega_{4}, 0 \leq C^{Y}(t) \leq \Omega_{5}\right\}
\end{aligned}
$$

Proposition 1 The compact set $\Theta$ is positively invariant for system (4).

Proof We have

$\left.\dot{S}\right|_{S=0}=\rho>0,\left.\quad \dot{L}\right|_{L=0}=(1-\beta) \eta_{1} S V \geq 0$ for all $S, V \geq 0$,

$\left.\dot{I}\right|_{I=0}=\beta \eta_{1} S V+\lambda L \geq 0$ for all $S, V, L \geq 0,\left.\quad \dot{E}\right|_{E=0}=\varphi \eta_{2} S Y+r Y$ for all $S, Y \geq 0$,

$\left.\dot{Y}\right|_{Y=0}=\psi E \geq 0$ for all $E \geq 0,\left.\quad \dot{V}\right|_{V=0}=b I \geq 0$ for all $I \geq 0$,

$\left.\dot{C}^{I}\right|_{C^{I}=0}=0,\left.\quad \dot{C}^{Y}\right|_{C^{Y}=0}=0$.

This insures that, $\left(S(t), L(t), I(t), E(t), Y(t), V(t), C^{I}(t), C^{Y}(t)\right) \in \mathbb{R}_{\geq 0}^{8}$ for all $t \geq 0$ when $\left(S(0), L(0), I(0), E(0), Y(0), V(0), C^{I}(0), C^{Y}(0)\right) \in \mathbb{R}_{\geq 0}^{8}$. To show the boundedness of all state variables, we let

$$
\Psi(t)=S+L+I+\frac{1}{\varphi}(E+Y)+\frac{a}{2 b} V+\frac{\mu_{1}}{\sigma_{1}} C^{I}+\frac{\mu_{2}}{\varphi \sigma_{2}} C^{Y} .
$$

Then

$$
\dot{\Psi}=\rho-\alpha S-\gamma L-\frac{a}{2} I-\frac{\omega}{\varphi} E-\frac{(\delta-r)}{\varphi} Y-\frac{a \varepsilon}{2 b} V-\frac{\mu_{1} \pi_{1}}{\sigma_{1}} C^{I}-\frac{\mu_{2} \pi_{2}}{\varphi \sigma_{2}} C^{Y} .
$$

We have $\delta-r=\delta^{*}-r^{*}>0$. Hence,

$$
\begin{aligned}
\dot{\Psi} & =\rho-\alpha S-\gamma L-\frac{a}{2} I-\frac{\omega}{\varphi} E-\frac{\left(\delta^{*}-r^{*}\right)}{\varphi} Y-\frac{a \varepsilon}{2 b} V-\frac{\mu_{1} \pi_{1}}{\sigma_{1}} C^{I}-\frac{\mu_{2} \pi_{2}}{\varphi \sigma_{2}} C^{Y} \\
& \leq \rho-\phi\left[S+L+I+\frac{1}{\varphi}(E+Y)+\frac{a}{2 b} V+\frac{\mu_{1}}{\sigma_{1}} C^{I}+\frac{\mu_{2}}{\varphi \sigma_{2}} C^{Y}\right]=\rho-\phi \Psi,
\end{aligned}
$$

where $\phi=\min \left\{\alpha, \gamma, \frac{a}{2}, \omega, \delta^{*}-r^{*}, \varepsilon, \pi_{1}, \pi_{2}\right\}$. Hence, $0 \leq \Psi(t) \leq \Omega_{1}$ if $\Psi(0) \leq \Omega_{1}$ for $t \geq 0$, where $\Omega_{1}=\frac{\rho}{\phi}$. Since $S, L, I, E, Y, V, C^{I}$, and $C^{Y}$ are all nonnegative then $0 \leq S(t), L(t), I(t) \leq \Omega_{1}, \quad 0 \leq E(t), Y(t) \leq \Omega_{2}, \quad 0 \leq V(t) \leq \Omega_{3}, \quad 0 \leq C^{I}(t) \leq \Omega_{4}$, $0 \leq C^{Y}(t) \leq \Omega_{5} \quad$ if $\quad S(0)+L(0)+I(0)+\frac{1}{\varphi}(E(0)+\quad Y(0))+\frac{a}{2 b} V(0)+\frac{\mu_{1}}{\sigma_{1}} C^{I}(0)+$ $\frac{\mu_{2}}{\varphi \sigma_{2}} C^{Y}(0) \leq \Omega_{1}$, where $\Omega_{2}=\varphi \Omega_{1}, \Omega_{3}=\frac{2 b \Omega_{1}}{a}, \Omega_{4}=\frac{\sigma_{1} \Omega_{1}}{\mu_{1}}$ and $\Omega_{5}=\frac{\varphi \sigma_{2} \Omega_{1}}{\mu_{2}}$.

\section{Threshold parameters and equilibria}

In this section, we derive eight threshold parameters which guarantee the existence of the equilibria of the model. Let $\left(S, L, I, E, Y, V, C^{I}, C^{Y}\right)$ be any equilibrium of system (4) satisfying the following equations: 


$$
\begin{gathered}
0=\rho-\alpha S-\eta_{1} S V-\eta_{2} S Y, \\
0=(1-\beta) \eta_{1} S V-(\lambda+\gamma) L, \\
0=\beta \eta_{1} S V+\lambda L-a I-\mu_{1} C^{I} I, \\
0=\varphi \eta_{2} S Y+r Y-(\psi+\omega) E \\
0=\psi E-\delta Y-\mu_{2} C^{Y} Y \\
0=b I-\varepsilon V \\
0=\left(\sigma_{1} I-\pi_{1}\right) C^{I} \\
0=\left(\sigma_{2} Y-\pi_{2}\right) C^{Y}
\end{gathered}
$$

The straightforward calculation finds that system (4) admits eight equilibria.

(1) Infection-free equilibrium, $\bigoplus_{0}=\left(S_{0}, 0,0,0,0,0,0,0\right)$, where $S_{0}=\rho / \alpha$. This case describes the situation of healthy state where both HIV and HTLV-I are absent.

(2) Chronic HIV mono-infection equilibrium with inactive immune response, $\bigoplus_{1}=\left(S_{1}, L_{1}, I_{1}, 0,0, V_{1}, 0,0\right)$, where

$$
\begin{aligned}
& S_{1}=\frac{a \varepsilon(\gamma+\lambda)}{\eta_{1} b(\beta \gamma+\lambda)}, L_{1}=\frac{a \varepsilon \alpha(1-\beta)}{\eta_{1} b(\beta \gamma+\lambda)}\left[\frac{\eta_{1} b S_{0}(\beta \gamma+\lambda)}{a \varepsilon(\gamma+\lambda)}-1\right], \\
& I_{1}=\frac{\varepsilon \alpha}{\eta_{1} b}\left[\frac{\eta_{1} b S_{0}(\beta \gamma+\lambda)}{a \varepsilon(\gamma+\lambda)}-1\right], V_{1}=\frac{\alpha}{\eta_{1}}\left[\frac{\eta_{1} b S_{0}(\beta \gamma+\lambda)}{a \varepsilon(\gamma+\lambda)}-1\right] .
\end{aligned}
$$

Therefore, $\bigoplus_{1}$ exists when

$$
\frac{\eta_{1} b S_{0}(\beta \gamma+\lambda)}{a \varepsilon(\gamma+\lambda)}>1
$$

At the equilibrium $\bigoplus_{1}$ the chronic HIV mono-infection persists while the immune response is unstimulated. The basic HIV mono-infection reproductive ratio for system (4) is defined as:

$$
\mathfrak{R}_{1}=\frac{\eta_{1} b S_{0}(\beta \gamma+\lambda)}{a \varepsilon(\gamma+\lambda)} .
$$

The parameter $\mathfrak{R}_{1}$ determines whether or not a chronic HIV infection can be established. In terms of $\mathfrak{R}_{1}$, we can write

$$
S_{1}=\frac{S_{0}}{\mathfrak{R}_{1}}, L_{1}=\frac{a \varepsilon \alpha(1-\beta)}{\eta_{1} b(\beta \gamma+\lambda)}\left(\Re_{1}-1\right), I_{1}=\frac{\varepsilon \alpha}{\eta_{1} b}\left(\Re_{1}-1\right), V_{1}=\frac{\alpha}{\eta_{1}}\left(\Re_{1}-1\right) .
$$

(3) Chronic HTLV mono-infection equilibrium with inactive immune response, $\mathrm{Ð}_{2}=\left(S_{2}, 0,0, E_{2}, Y_{2}, 0,0,0\right)$, where 


$$
\begin{aligned}
& S_{2}=\frac{(\delta-r) \psi+\delta \omega}{\varphi \eta_{2} \psi}, \quad E_{2}=\frac{\alpha \delta}{\eta_{2} \psi}\left[\frac{\varphi \eta_{2} \psi S_{0}}{(\delta-r) \psi+\delta \omega}-1\right] \\
& Y_{2}=\frac{\alpha}{\eta_{2}}\left[\frac{\varphi \eta_{2} \psi S_{0}}{(\delta-r) \psi+\delta \omega}-1\right] .
\end{aligned}
$$

Therefore, $\mathrm{Ð}_{2}$ exists when

$$
\frac{\varphi \eta_{2} \psi S_{0}}{(\delta-r) \psi+\delta \omega}>1
$$

At the equilibrium $\mathrm{Ð}_{2}$ the chronic HTLV mono-infection persists while the immune response is unstimulated. The basic HTLV mono-infection reproductive ratio for system (4) is defined as:

$$
\mathfrak{R}_{2}=\frac{\varphi \eta_{2} \psi S_{0}}{(\delta-r) \psi+\delta \omega} .
$$

The parameter $\mathfrak{R}_{2}$ decides whether or not a chronic HTLV infection can be established. In terms of $\mathfrak{R}_{2}$, we can write

$$
S_{2}=\frac{S_{0}}{\mathfrak{R}_{2}}, \quad E_{2}=\frac{\alpha \delta}{\eta_{2} \psi}\left(\mathfrak{R}_{2}-1\right), \quad Y_{2}=\frac{\alpha}{\eta_{2}}\left(\mathfrak{R}_{2}-1\right)
$$

(4) Chronic HIV mono-infection equilibrium with only active HIV-specific CTL, $\bigoplus_{3}=\left(S_{3}, L_{3}, I_{3}, 0,0, V_{3}, C_{3}^{I}, 0\right)$, where

$$
\begin{aligned}
& S_{3}=\frac{\varepsilon \sigma_{1} \rho}{\pi_{1} \eta_{1} b+\alpha \varepsilon \sigma_{1}}, \quad L_{3}=\frac{\pi_{1} \eta_{1} b \rho(1-\beta)}{(\gamma+\lambda)\left(\pi_{1} \eta_{1} b+\alpha \varepsilon \sigma_{1}\right)}, \quad I_{3}=\frac{\pi_{1}}{\sigma_{1}}, \\
& V_{3}=\frac{b}{\varepsilon} I_{3}=\frac{b \pi_{1}}{\varepsilon \sigma_{1}}, \quad C_{3}^{I}=\frac{a}{\mu_{1}}\left[\frac{\sigma_{1} \rho \eta_{1} b(\beta \gamma+\lambda)}{a(\gamma+\lambda)\left(\pi_{1} \eta_{1} b+\alpha \varepsilon \sigma_{1}\right)}-1\right] .
\end{aligned}
$$

We note that $\mathrm{Ð}_{3}$ exists when $\frac{\sigma_{1} \rho \eta_{1} b(\beta \gamma+\lambda)}{a(\gamma+\lambda)\left(\pi_{1} \eta_{1} b+\alpha \varepsilon \sigma_{1}\right)}>1$. The HIV-specific CTLmediated immunity reproductive ratio in case of HIV mono-infection is stated as:

$$
\mathfrak{R}_{3}=\frac{\sigma_{1} \rho \eta_{1} b(\beta \gamma+\lambda)}{a(\gamma+\lambda)\left(\pi_{1} \eta_{1} b+\alpha \varepsilon \sigma_{1}\right)} .
$$

Thus, $C_{3}^{I}=\frac{a}{\mu_{1}}\left(\mathfrak{R}_{3}-1\right)$. The parameter $\mathfrak{R}_{3}$ determines whether or not the HIVspecific CTL-mediated immune response is stimulated in the absent of HTLV infection.

(5) Chronic HTLV mono-infection equilibrium with only active HTLV-specific $\mathrm{CTL}, \mathrm{Ð}_{4}=\left(S_{4}, 0,0, E_{4}, Y_{4}, 0,0, C_{4}^{Y}\right)$, where 


$$
\begin{aligned}
S_{4} & =\frac{\sigma_{2} \rho}{\pi_{2} \eta_{2}+\alpha \sigma_{2}}, \quad Y_{4}=\frac{\pi_{2}}{\sigma_{2}}, \quad E_{4}=\frac{\pi_{2}\left[r\left(\pi_{2} \eta_{2}+\alpha \sigma_{2}\right)+\eta_{2} \rho \varphi \sigma_{2}\right]}{\sigma_{2}(\psi+\omega)\left(\pi_{2} \eta_{2}+\alpha \sigma_{2}\right)}, \\
C_{4}^{Y} & =\frac{(\delta-r) \psi+\delta \omega}{\mu_{2}(\psi+\omega)}\left[\frac{\psi \sigma_{2} \rho \varphi \eta_{2}}{\{(\delta-r) \psi+\delta \omega\}\left(\pi_{2} \eta_{2}+\alpha \sigma_{2}\right)}-1\right] .
\end{aligned}
$$

We note that $\mathrm{Ð}_{4}$ exists when $\frac{\psi \sigma_{2} \rho \varphi \eta_{2}}{[(\delta-r) \psi+\delta \omega]\left(\pi_{2} \eta_{2}+\alpha \sigma_{2}\right)}>1$. The HTLVspecific CTL-mediated immunity reproductive ratio in case of HTLV mono-infection is stated as:

$$
\mathfrak{R}_{4}=\frac{\psi \sigma_{2} \rho \varphi \eta_{2}}{[(\delta-r) \psi+\delta \omega]\left(\pi_{2} \eta_{2}+\alpha \sigma_{2}\right)}
$$

Thus, $C_{4}^{Y}=\frac{(\delta-r) \psi+\delta \omega}{\mu_{2}(\psi+\omega)}\left(\mathfrak{R}_{4}-1\right)$. The parameter $\mathfrak{R}_{4}$ determines whether or not the HTLV-specific CTL-mediated immune response is stimulated in the absent of HIV infection.

(6) Chronic HIV/HTLV co-infection equilibrium with only active HIV-specific $\mathrm{CTL}, \mathrm{Ð}_{5}=\left(S_{5}, L_{5}, I_{5}, E_{5}, Y_{5}, V_{5}, C_{5}^{I}, 0\right)$, where

$$
\begin{aligned}
S_{5} & =\frac{(\delta-r) \psi+\delta \omega}{\varphi \eta_{2} \psi}=S_{2}, \quad I_{5}=\frac{\pi_{1}}{\sigma_{1}}=I_{3}, \\
V_{5} & =\frac{b \pi_{1}}{\varepsilon \sigma_{1}}=V_{3}, \quad L_{5}=\frac{\pi_{1} \eta_{1} b(1-\beta)[(\delta-r) \psi+\delta \omega]}{\varepsilon \eta_{2} \sigma_{1} \varphi \psi(\gamma+\lambda)}, \\
E_{5} & =\frac{\delta\left(\pi_{1} \eta_{1} b+\alpha \varepsilon \sigma_{1}\right)}{\varepsilon \eta_{2} \sigma_{1} \psi}\left[\frac{\rho \varphi \varepsilon \eta_{2} \sigma_{1} \psi}{\{(\delta-r) \psi+\delta \omega\}\left(\pi_{1} \eta_{1} b+\alpha \varepsilon \sigma_{1}\right)}-1\right] \\
Y_{5} & =\frac{\pi_{1} \eta_{1} b+\alpha \varepsilon \sigma_{1}}{\varepsilon \eta_{2} \sigma_{1}}\left[\frac{\rho \varphi \varepsilon \eta_{2} \sigma_{1} \psi}{\{(\delta-r) \psi+\delta \omega\}\left(\pi_{1} \eta_{1} b+\alpha \varepsilon \sigma_{1}\right)}-1\right] \\
C_{5}^{I} & =\frac{a}{\mu_{1}}\left[\frac{\eta_{1} b(\beta \gamma+\lambda)\{(\delta-r) \psi+\delta \omega\}}{a \varepsilon \varphi \eta_{2} \psi(\gamma+\lambda)}-1\right]=\frac{a}{\mu_{1}}\left(\mathfrak{R}_{1} / \mathfrak{R}_{2}-1\right) .
\end{aligned}
$$

We note that $\bigoplus_{5}$ exists when $\mathfrak{R}_{1} / \mathfrak{R}_{2}>1$ and $\frac{\rho \varphi \varepsilon \eta_{2} \sigma_{1} \psi}{[(\delta-r) \psi+\delta \omega]\left(\pi_{1} \eta_{1} b+\alpha \varepsilon \sigma_{1}\right)}>1$. The HTLV infection reproductive ratio in the presence of HIV infection is stated as:

$$
\mathfrak{R}_{5}=\frac{\rho \varphi \varepsilon \eta_{2} \sigma_{1} \psi}{[(\delta-r) \psi+\delta \omega]\left(\pi_{1} \eta_{1} b+\alpha \varepsilon \sigma_{1}\right)} .
$$

The parameter $\mathfrak{R}_{5}$ determines whether or not HIV-infected patients could be coinfected with HTLV. Thus, $E_{5}=\frac{\delta\left(\pi_{1} \eta_{1} b+\alpha \varepsilon \sigma_{1}\right)}{\varepsilon \eta_{2} \sigma_{1} \psi}\left(\mathfrak{R}_{5}-1\right)$, $Y_{5}=\frac{\pi_{1} \eta_{1} b+\alpha \varepsilon \sigma_{1}}{\varepsilon \eta_{2} \sigma_{1}}\left(\Re_{5}-1\right)$

(7) Chronic HIV/HTLV co-infection equilibrium with only active HTLV-specific CTL, $Ð_{6}=\left(S_{6}, L_{6}, I_{6}, E_{6}, Y_{6}, V_{6}, 0, C_{6}^{Y}\right)$, where 


$$
\begin{aligned}
S_{6} & =\frac{a \varepsilon(\gamma+\lambda)}{\eta_{1} b(\beta \gamma+\lambda)}=S_{1}, \\
L_{6} & =\frac{a \varepsilon(1-\beta)\left(\pi_{2} \eta_{2}+\alpha \sigma_{2}\right)}{b \eta_{1} \sigma_{2}(\beta \gamma+\lambda)}\left[\frac{\rho b \eta_{1} \sigma_{2}(\beta \gamma+\lambda)}{a \varepsilon(\gamma+\lambda)\left(\pi_{2} \eta_{2}+\alpha \sigma_{2}\right)}-1\right], \\
I_{6} & =\frac{\varepsilon\left(\pi_{2} \eta_{2}+\alpha \sigma_{2}\right)}{b \eta_{1} \sigma_{2}}\left[\frac{\rho b \eta_{1} \sigma_{2}(\beta \gamma+\lambda)}{a \varepsilon(\gamma+\lambda)\left(\pi_{2} \eta_{2}+\alpha \sigma_{2}\right)}-1\right], \\
E_{6} & =\frac{\pi_{2}\left[b r \eta_{1}(\beta \gamma+\lambda)+a \varepsilon \varphi \eta_{2}(\gamma+\lambda)\right]}{b \eta_{1} \sigma_{2}(\beta \gamma+\lambda)(\psi+\omega)}, \\
Y_{6} & =\frac{\pi_{2}}{\sigma_{2}}=Y_{4}, \quad V_{6}=\frac{\pi_{2} \eta_{2}+\alpha \sigma_{2}}{\eta_{1} \sigma_{2}}\left[\frac{\rho b \eta_{1} \sigma_{2}(\beta \gamma+\lambda)}{a \varepsilon(\gamma+\lambda)\left(\pi_{2} \eta_{2}+\alpha \sigma_{2}\right)}-1\right], \\
C_{6}^{Y} & =\frac{(\delta-r) \psi+\delta \omega}{\mu_{2}(\psi+\omega)}\left[\frac{a \varepsilon \varphi \eta_{2} \psi(\gamma+\lambda)}{\eta_{1} b(\beta \gamma+\lambda)\{(\delta-r) \psi+\delta \omega\}}-1\right] \\
& =\frac{(\delta-r) \psi+\delta \omega}{\mu_{2}(\psi+\omega)}\left(\mathfrak{R}_{2} / \mathfrak{R}_{1}-1\right) .
\end{aligned}
$$

We note that $\bigoplus_{6}$ exists when $\mathfrak{R}_{2} / \mathfrak{R}_{1}>1$ and $\frac{\rho b \eta_{1} \sigma_{2}(\beta \gamma+\lambda)}{a \varepsilon(\gamma+\lambda)\left(\pi_{2} \eta_{2}+\alpha \sigma_{2}\right)}>1$. The HIV infection reproductive ratio in the presence of HTLV infection is stated as:

$$
\mathfrak{R}_{6}=\frac{\rho b \eta_{1} \sigma_{2}(\beta \gamma+\lambda)}{a \varepsilon(\gamma+\lambda)\left(\pi_{2} \eta_{2}+\alpha \sigma_{2}\right)} .
$$

Thus, $\quad L_{6}=\frac{a \varepsilon(1-\beta)\left(\pi_{2} \eta_{2}+\alpha \sigma_{2}\right)}{b \eta_{1} \sigma_{2}(\beta \gamma+\lambda)}\left(\mathfrak{R}_{6}-1\right), \quad I_{6}=\frac{\varepsilon\left(\pi_{2} \eta_{2}+\alpha \sigma_{2}\right)}{b \eta_{1} \sigma_{2}}\left(\mathfrak{R}_{6}-1\right)$, $V_{6}=\frac{\pi_{2} \eta_{2}+\alpha \sigma_{2}}{\eta_{1} \sigma_{2}}\left(\mathfrak{R}_{6}-1\right)$. The parameter $\mathfrak{R}_{6}$ determines whether or not HTLVinfected patients could be co-infected with HIV.

(8) Chronic HIV/HTLV co-infection equilibrium with active HIV-specific CTL and HTLV-specific CTL, $\bigoplus_{7}=\left(S_{7}, L_{7}, I_{7}, E_{7}, Y_{7}, V_{7}, C_{7}^{I}, C_{7}^{Y}\right)$, where

$$
\begin{aligned}
S_{7} & =\frac{\varepsilon \sigma_{1} \sigma_{2} \rho}{\pi_{1} \eta_{1} b \sigma_{2}+\pi_{2} \eta_{2} \varepsilon \sigma_{1}+\alpha \varepsilon \sigma_{1} \sigma_{2}}, \\
L_{7} & =\frac{\pi_{1} \eta_{1} b \sigma_{2} \rho(1-\beta)}{(\gamma+\lambda)\left(\pi_{1} \eta_{1} b \sigma_{2}+\pi_{2} \eta_{2} \varepsilon \sigma_{1}+\alpha \varepsilon \sigma_{1} \sigma_{2}\right)}, \\
E_{7} & =\frac{\pi_{2}\left[r\left(\pi_{1} \eta_{1} b \sigma_{2}+\pi_{2} \eta_{2} \varepsilon \sigma_{1}+\alpha \varepsilon \sigma_{1} \sigma_{2}\right)+\eta_{2} \varepsilon \sigma_{1} \sigma_{2} \rho \varphi\right]}{\sigma_{2}(\psi+\omega)\left(\pi_{1} \eta_{1} b \sigma_{2}+\pi_{2} \eta_{2} \varepsilon \sigma_{1}+\alpha \varepsilon \sigma_{1} \sigma_{2}\right)}, \\
I_{7} & =\frac{\pi_{1}}{\sigma_{1}}=I_{3}=I_{5}, \quad Y_{7}=\frac{\pi_{2}}{\sigma_{2}}=Y_{4}=Y_{6}, \quad V_{7}=\frac{b \pi_{1}}{\varepsilon \sigma_{1}}=V_{3}=V_{5}, \\
C_{7}^{I} & =\frac{a}{\mu_{1}}\left[\frac{\eta_{1} b \sigma_{1} \sigma_{2} \rho(\beta \gamma+\lambda)}{a(\gamma+\lambda)\left(\pi_{1} \eta_{1} b \sigma_{2}+\pi_{2} \eta_{2} \varepsilon \sigma_{1}+\alpha \varepsilon \sigma_{1} \sigma_{2}\right)}-1\right], \\
C_{7}^{Y} & =\frac{(\delta-r) \psi+\delta \omega}{\mu_{2}(\psi+\omega)}\left[\frac{\psi\left(\eta_{2} \varepsilon \sigma_{1} \sigma_{2} \rho\right.}{\{(\delta-r) \psi+\delta \omega)\}\left(\pi_{1} \eta_{1} b \sigma_{2}+\pi_{2} \eta_{2} \varepsilon \sigma_{1}+\alpha \varepsilon \sigma_{1} \sigma_{2}\right)}-1\right] .
\end{aligned}
$$

It is obvious that $\mathrm{Ð}_{7}$ exists when $\frac{\eta_{1} b \sigma_{1} \sigma_{2} \rho(\beta \gamma+\lambda)}{a(\gamma+\lambda)\left(\pi_{1} \eta_{1} b \sigma_{2}+\pi_{2} \eta_{2} \varepsilon \sigma_{1}+\alpha \varepsilon \sigma_{1} \sigma_{2}\right)}>1$ and 


$$
\frac{\psi \varphi \eta_{2} \varepsilon \sigma_{1} \sigma_{2} \rho}{[(\delta-r) \psi+\delta \omega)]\left(\pi_{1} \eta_{1} b \sigma_{2}+\pi_{2} \eta_{2} \varepsilon \sigma_{1}+\alpha \varepsilon \sigma_{1} \sigma_{2}\right)}>1 \text {. Now we define }
$$

$$
\begin{aligned}
\mathfrak{R}_{7} & =\frac{\eta_{1} b \sigma_{1} \sigma_{2} \rho(\beta \gamma+\lambda)}{a(\gamma+\lambda)\left(\pi_{1} \eta_{1} b \sigma_{2}+\pi_{2} \eta_{2} \varepsilon \sigma_{1}+\alpha \varepsilon \sigma_{1} \sigma_{2}\right)}, \\
\mathfrak{R}_{8} & =\frac{\psi \varphi \eta_{2} \varepsilon \sigma_{1} \sigma_{2} \rho}{[(\delta-r) \psi+\delta \omega)]\left(\pi_{1} \eta_{1} b \sigma_{2}+\pi_{2} \eta_{2} \varepsilon \sigma_{1}+\alpha \varepsilon \sigma_{1} \sigma_{2}\right)} .
\end{aligned}
$$

Clearly, $\bigoplus_{7}$ exists when $\mathfrak{R}_{7}>1$ and $\mathfrak{R}_{8}>1$ and we can write $C_{7}^{I}=\frac{a}{\mu_{1}}\left(\mathfrak{R}_{7}-1\right)$ and $C_{7}^{Y}=\frac{(\delta-r) \psi+\delta \omega}{\mu_{2}(\psi+\omega)}\left(\mathfrak{R}_{8}-1\right)$. The parameter $\mathfrak{R}_{7}$ refers to the competed HIVspecific CTL-mediated immunity reproductive ratio in case of HIV/HTLV co-infection. On the other hand, the parameter $\mathfrak{R}_{8}$ refers to the competed HTLV-specific CTL-mediated immunity reproductive ratio in case of HIV/HTLV co-infection.

The eight threshold parameters are given as follows:

$$
\begin{aligned}
& \mathfrak{R}_{1}=\frac{\eta_{1} b S_{0}(\beta \gamma+\lambda)}{a \varepsilon(\gamma+\lambda)}, \quad \mathfrak{R}_{2}=\frac{\varphi \eta_{2} \psi S_{0}}{(\delta-r) \psi+\delta \omega}, \quad \mathfrak{R}_{3}=\frac{\sigma_{1} \rho \eta_{1} b(\beta \gamma+\lambda)}{a(\gamma+\lambda)\left(\pi_{1} \eta_{1} b+\alpha \varepsilon \sigma_{1}\right)} \\
& \mathfrak{R}_{4}=\frac{\psi \sigma_{2} \rho \varphi \eta_{2}}{[(\delta-r) \psi+\delta \omega]\left(\pi_{2} \eta_{2}+\alpha \sigma_{2}\right)}, \quad \mathfrak{R}_{5}=\frac{\rho \varphi \varepsilon \eta_{2} \sigma_{1} \psi}{[(\delta-r) \psi+\delta \omega]\left(\pi_{1} \eta_{1} b+\alpha \varepsilon \sigma_{1}\right)} \\
& \mathfrak{R}_{6}=\frac{\rho b \eta_{1} \sigma_{2}(\beta \gamma+\lambda)}{a \varepsilon(\gamma+\lambda)\left(\pi_{2} \eta_{2}+\alpha \sigma_{2}\right)}, \quad \mathfrak{R}_{7}=\frac{\eta_{1} b \sigma_{1} \sigma_{2} \rho(\beta \gamma+\lambda)}{a(\gamma+\lambda)\left(\pi_{1} \eta_{1} b \sigma_{2}+\pi_{2} \eta_{2} \varepsilon \sigma_{1}+\alpha \varepsilon \sigma_{1} \sigma_{2}\right)} \\
& \mathfrak{R}_{8}=\frac{\psi \varphi \eta_{2} \varepsilon \sigma_{1} \sigma_{2} \rho}{[(\delta-r) \psi+\delta \omega)]\left(\pi_{1} \eta_{1} b \sigma_{2}+\pi_{2} \eta_{2} \varepsilon \sigma_{1}+\alpha \varepsilon \sigma_{1} \sigma_{2}\right)}
\end{aligned}
$$

According to the above discussion, we sum up the existence conditions for all equilibria in Table 2 .

\section{Global stability analysis}

In this section, we prove the global asymptotic stability of all equilibria by constructing Lyapunov function and applying Lyapunov-LaSalle asymptotic stability theorem [54-56]. We will use the arithmetic-geometric mean inequality

$$
\frac{1}{n} \sum_{i=1}^{n} \chi_{i} \geq \sqrt[n]{\prod_{i=1}^{n} \chi_{i}}, \quad \chi_{i} \geq 0, i=1,2, \ldots
$$

which yields

$$
\frac{S_{j}}{S}+\frac{S V I_{j}}{S_{j} V_{j} I}+\frac{I V_{j}}{I_{j} V} \geq 3, \quad j=1,3,5,6,7,
$$


Table 2 Model (4) equilibria and their existence conditions

\begin{tabular}{|c|c|c|}
\hline Equilibrium point & Definition & $\begin{array}{l}\text { Existence } \\
\text { conditions }\end{array}$ \\
\hline $\mathrm{Ð}_{0}=\left(S_{0}, 0,0,0,0,0,0,0\right)$ & Infection-free equilibrium & None \\
\hline$\bigoplus_{1}=\left(S_{1}, L_{1}, I_{1}, 0,0, V_{1}, 0,0\right)$ & $\begin{array}{l}\text { Chronic HIV mono-infection equilibrium with } \\
\text { inactive immune response }\end{array}$ & $\mathfrak{R}_{1}>1$ \\
\hline $\mathrm{Ð}_{2}=\left(S_{2}, 0,0, E_{2}, Y_{2}, 0,0,0\right)$ & $\begin{array}{l}\text { Chronic HTLV mono-infection equilibrium with } \\
\text { inactive immune response }\end{array}$ & $\mathfrak{R}_{2}>1$ \\
\hline$\bigoplus_{3}=\left(S_{3}, L_{3}, I_{3}, 0,0, V_{3}, C_{3}^{I}, 0\right)$ & $\begin{array}{l}\text { Chronic HIV mono-infection equilibrium with } \\
\text { only active HIV-specific CTL }\end{array}$ & $\mathfrak{R}_{3}>1$ \\
\hline $\mathrm{Ð}_{4}=\left(S_{4}, 0,0, E_{4}, Y_{4}, 0,0, C_{4}^{Y}\right)$ & $\begin{array}{l}\text { Chronic HTLV mono-infection equilibrium with } \\
\text { only active HTLV-specific CTL }\end{array}$ & $\mathfrak{R}_{4}>1$ \\
\hline $\mathrm{Ð}_{5}=\left(S_{5}, L_{5}, I_{5}, E_{5}, Y_{5}, V_{5}, C_{5}^{I}, 0\right)$ & $\begin{array}{l}\text { Chronic HIV/HTLV co-infection equilibrium with } \\
\text { only active HIV-specific CTL }\end{array}$ & $\begin{array}{l}\mathfrak{R}_{5}>1 \text { and } \\
\mathfrak{R}_{1} / \mathfrak{R}_{2}>1\end{array}$ \\
\hline $\mathrm{Ð}_{6}=\left(S_{6}, L_{6}, I_{6}, E_{6}, Y_{6}, V_{6}, 0, C_{6}^{Y}\right)$ & $\begin{array}{l}\text { Chronic HIV/HTLV co-infection equilibrium with } \\
\text { only active HTLV-specific CTL }\end{array}$ & $\begin{array}{l}\mathfrak{R}_{6}>1 \text { and } \\
\mathfrak{R}_{2} / \mathfrak{R}_{1}>1\end{array}$ \\
\hline $\mathrm{Ð}_{7}=\left(S_{7}, L_{7}, I_{7}, E_{7}, Y_{7}, V_{7}, C_{7}^{I}, C_{7}^{Y}\right)$ & $\begin{array}{l}\text { Chronic HIV/HTLV co-infection equilibrium with } \\
\text { active HIV-specific CTL and HTLV-specific } \\
\text { CTL }\end{array}$ & $\begin{array}{l}\mathfrak{R}_{7}>1 \text { and } \\
\mathfrak{R}_{8}>1\end{array}$ \\
\hline
\end{tabular}

$$
\begin{gathered}
\frac{S_{j}}{S}+\frac{S V L_{j}}{S_{j} V_{j} L}+\frac{L I_{j}}{L_{j} I}+\frac{I V_{j}}{I_{j} V} \geq 4, \quad j=1,3,5,6,7, \\
\frac{S_{j}}{S}+\frac{S Y E_{j}}{S_{j} Y_{j} E}+\frac{E Y_{j}}{E_{j} Y} \geq 3, \quad j=2,4,5,6,7 .
\end{gathered}
$$

Let a function $\Phi_{j}\left(S, L, I, E, Y, V, C^{I}, C^{Y}\right)$ and $\Upsilon_{j}^{\prime}$ be the largest invariant subset of

$$
\Upsilon_{j}=\left\{\left(S, L, I, E, Y, V, C^{I}, C^{Y}\right): \frac{\mathrm{d} \Phi_{j}}{\mathrm{~d} t}=0\right\}, j=0,1,2, \ldots, 7 .
$$

We define a function

$$
F(v)=v-1-\ln v .
$$

Theorem 1 If $\mathfrak{R}_{1} \leq 1$ and $\mathfrak{R}_{2} \leq 1$, then $\bigoplus_{0}$ is globally asymptotically stable (G.A.S).

Proof Constructing a Lyapunov function candidate $\Phi_{0}\left(S, L, I, E, Y, V, C^{I}, C^{Y}\right)$ :

$$
\begin{aligned}
\Phi_{0}= & S_{0} F\left(\frac{S}{S_{0}}\right)+\frac{\lambda}{\beta \gamma+\lambda} L+\frac{\gamma+\lambda}{\beta \gamma+\lambda} I+\frac{1}{\varphi} E+\frac{\psi+\omega}{\varphi \psi} Y \\
& +\frac{a(\gamma+\lambda)}{b(\beta \gamma+\lambda)} V+\frac{\mu_{1}(\gamma+\lambda)}{\sigma_{1}(\beta \gamma+\lambda)} C^{I}+\frac{\mu_{2}(\psi+\omega)}{\varphi \psi \sigma_{2}} C^{Y} .
\end{aligned}
$$

It is seen that, $\Phi_{0}\left(S, L, I, E, Y, V, C^{I}, C^{Y}\right)>0$ for all $S, L, I, E, Y, V, C^{I}, C^{Y}>0$, and 
$\Phi_{0}$ has a global minimum at $\bigoplus_{0}$. We calculate $\frac{\mathrm{d} \Phi_{0}}{\mathrm{~d} t}$ along the solutions of model (4) as:

$$
\begin{aligned}
\frac{\mathrm{d} \Phi_{0}}{\mathrm{~d} t}= & \left(1-\frac{S_{0}}{S}\right)\left[\rho-\alpha S-\eta_{1} S V-\eta_{2} S Y\right]+\frac{\lambda}{\beta \gamma+\lambda}\left[(1-\beta) \eta_{1} S V-(\lambda+\gamma) L\right] \\
& +\frac{\gamma+\lambda}{\beta \gamma+\lambda}\left[\beta \eta_{1} S V+\lambda L-a I-\mu_{1} C^{I} I\right]+\frac{1}{\varphi}\left[\varphi \eta_{2} S Y+r Y-(\psi+\omega) E\right] \\
& +\frac{\psi+\omega}{\varphi \psi}\left[\psi E-\delta Y-\mu_{2} C^{Y} Y\right]+\frac{a(\gamma+\lambda)}{b(\beta \gamma+\lambda)}[b I-\varepsilon V] \\
& +\frac{\mu_{1}(\gamma+\lambda)}{\sigma_{1}(\beta \gamma+\lambda)}\left[\sigma_{1} C^{I} I-\pi_{1} C^{I}\right]+\frac{\mu_{2}(\psi+\omega)}{\varphi \psi \sigma_{2}}\left[\sigma_{2} C^{Y} Y-\pi_{2} C^{Y}\right] \\
= & \left(1-\frac{S_{0}}{S}\right)(\rho-\alpha S)+\eta_{1} S_{0} V+\eta_{2} S_{0} Y+\frac{r}{\varphi} Y-\frac{\delta(\psi+\omega)}{\varphi \psi} Y-\frac{a \varepsilon(\gamma+\lambda)}{b(\beta \gamma+\lambda)} V \\
& -\frac{\mu_{1} \pi_{1}(\gamma+\lambda)}{\sigma_{1}(\beta \gamma+\lambda)} C^{I}-\frac{\mu_{2} \pi_{2}(\psi+\omega)}{\varphi \psi \sigma_{2}} C^{Y} .
\end{aligned}
$$

Using $S_{0}=\rho / \alpha$, we obtain

$$
\begin{aligned}
\frac{\mathrm{d} \Phi_{0}}{\mathrm{~d} t}= & -\alpha \frac{\left(S-S_{0}\right)^{2}}{S}+\frac{a \varepsilon(\gamma+\lambda)}{b(\beta \gamma+\lambda)}\left(\mathfrak{R}_{1}-1\right) V+\frac{(\delta-r) \psi+\delta \omega}{\varphi \psi}\left(\mathfrak{R}_{2}-1\right) Y \\
& -\frac{\mu_{1} \pi_{1}(\gamma+\lambda)}{\sigma_{1}(\beta \gamma+\lambda)} C^{I}-\frac{\mu_{2} \pi_{2}(\psi+\omega)}{\varphi \psi \sigma_{2}} C^{Y} .
\end{aligned}
$$

Therefore, $\frac{\mathrm{d} \Phi_{0}}{\mathrm{~d} t} \leq 0$ for all $S, Y, V, C^{I}, C^{Y}>0$, where $\frac{\mathrm{d} \Phi_{0}}{\mathrm{~d} t}=0$ occurs at $S=S_{0}$ and $V=Y=C^{I}=C^{Y}=0$. The solutions of system (4) are confined to $\Upsilon_{0}^{\prime}$. The set $\Upsilon_{0}^{\prime}$ contains elements with $Y=V=0$ and then $\dot{Y}=\dot{V}=0$. The fifth and sixth equations of system (4) imply

$$
\begin{aligned}
& 0=\dot{Y}=\psi E, \\
& 0=\dot{V}=b I .
\end{aligned}
$$

Hence, $E(t)=I(t)=0$ for all $t$. In addition, from the third equation of system (4) we have

$$
0=\dot{I}=\lambda L
$$

which yields $L(t)=0$ for all $t$. Therefore, $\Upsilon_{0}^{\prime}=\left\{\bigoplus_{0}\right\}$ and applying LyapunovLaSalle asymptotic stability theorem [54-56] we get that $\bigoplus_{0}$ is G.A.S.

Theorem 2 If $\mathfrak{R}_{1}>1, \mathfrak{R}_{2} / \mathfrak{R}_{1} \leq 1$ and $\mathfrak{R}_{3} \leq 1$, then $\emptyset_{1}$ is G.A.S.

Proof Define a function $\Phi_{1}\left(S, L, I, E, Y, V, C^{I}, C^{Y}\right)$ as: 


$$
\begin{aligned}
\Phi_{1}= & S_{1} F\left(\frac{S}{S_{1}}\right)+\frac{\lambda}{\beta \gamma+\lambda} L_{1} F\left(\frac{L}{L_{1}}\right)+\frac{\gamma+\lambda}{\beta \gamma+\lambda} I_{1} F\left(\frac{I}{I_{1}}\right)+\frac{1}{\varphi} E+\frac{\psi+\omega}{\varphi \psi} Y \\
& +\frac{a(\gamma+\lambda)}{b(\beta \gamma+\lambda)} V_{1} F\left(\frac{V}{V_{1}}\right)+\frac{\mu_{1}(\gamma+\lambda)}{\sigma_{1}(\beta \gamma+\lambda)} C^{I}+\frac{\mu_{2}(\psi+\omega)}{\varphi \psi \sigma_{2}} C^{Y} .
\end{aligned}
$$

Calculating $\frac{\mathrm{d} \Phi_{1}}{\mathrm{~d} t}$ as:

$$
\begin{aligned}
& \frac{\mathrm{d} \Phi_{1}}{\mathrm{~d} t}=\left(1-\frac{S_{1}}{S}\right)\left[\rho-\alpha S-\eta_{1} S V-\eta_{2} S Y\right]+\frac{\lambda}{\beta \gamma+\lambda}\left(1-\frac{L_{1}}{L}\right) \\
& \times\left[(1-\beta) \eta_{1} S V-(\lambda+\gamma) L\right] \\
& +\frac{\gamma+\lambda}{\beta \gamma+\lambda}\left(1-\frac{I_{1}}{I}\right)\left[\beta \eta_{1} S V+\lambda L-a I-\mu_{1} C^{I} I\right] \\
& +\frac{1}{\varphi}\left[\varphi \eta_{2} S Y+r Y-(\psi+\omega) E\right] \\
& +\frac{\psi+\omega}{\varphi \psi}\left[\psi E-\delta Y-\mu_{2} C^{Y} Y\right]+\frac{a(\gamma+\lambda)}{b(\beta \gamma+\lambda)}\left(1-\frac{V_{1}}{V}\right)[b I-\varepsilon V] \\
& +\frac{\mu_{1}(\gamma+\lambda)}{\sigma_{1}(\beta \gamma+\lambda)}\left[\sigma_{1} C^{I} I-\pi_{1} C^{I}\right]+\frac{\mu_{2}(\psi+\omega)}{\varphi \psi \sigma_{2}}\left[\sigma_{2} C^{Y} Y-\pi_{2} C^{Y}\right] \\
& =\left(1-\frac{S_{1}}{S}\right)(\rho-\alpha S)+\eta_{1} S_{1} V+\eta_{2} S_{1} Y-\frac{\lambda(1-\beta)}{\beta \gamma+\lambda} \eta_{1} S V \frac{L_{1}}{L}+\frac{\lambda(\gamma+\lambda)}{\beta \gamma+\lambda} L_{1} \\
& -\frac{\beta(\gamma+\lambda)}{\beta \gamma+\lambda} \eta_{1} S V \frac{I_{1}}{I}-\frac{\lambda(\gamma+\lambda)}{\beta \gamma+\lambda} L \frac{I_{1}}{I}+\frac{a(\gamma+\lambda)}{\beta \gamma+\lambda} I_{1}+\frac{\mu_{1}(\gamma+\lambda)}{\beta \gamma+\lambda} C^{I} I_{1} \\
& +\frac{r}{\varphi} Y-\frac{\delta(\psi+\omega)}{\varphi \psi} Y-\frac{a \varepsilon(\gamma+\lambda)}{b(\beta \gamma+\lambda)} V-\frac{a(\gamma+\lambda)}{\beta \gamma+\lambda} I \frac{V_{1}}{V}+\frac{a \varepsilon(\gamma+\lambda)}{b(\beta \gamma+\lambda)} V_{1} \\
& -\frac{\mu_{1} \pi_{1}(\gamma+\lambda)}{\sigma_{1}(\beta \gamma+\lambda)} C^{I}-\frac{\mu_{2} \pi_{2}(\psi+\omega)}{\varphi \psi \sigma_{2}} C^{Y} .
\end{aligned}
$$

Using the equilibrium conditions for $\bigoplus_{1}$, we get

$$
\begin{aligned}
& \rho=\alpha S_{1}+\eta_{1} S_{1} V_{1}, \quad \frac{\lambda(1-\beta)}{\beta \gamma+\lambda} \eta_{1} S_{1} V_{1}=\frac{\lambda(\gamma+\lambda)}{\beta \gamma+\lambda} L_{1}, \\
& \eta_{1} S_{1} V_{1}=\frac{a(\gamma+\lambda)}{\beta \gamma+\lambda} I_{1}=\frac{a \varepsilon(\gamma+\lambda)}{b(\beta \gamma+\lambda)} V_{1} .
\end{aligned}
$$

Then, we obtain 


$$
\begin{aligned}
& \frac{\mathrm{d} \Phi_{1}}{\mathrm{~d} t}=\left(1-\frac{S_{1}}{S}\right)\left(\alpha S_{1}-\alpha S\right)+\eta_{1} S_{1} V_{1}\left(1-\frac{S_{1}}{S}\right) \\
& +\eta_{2} S_{1} Y-\frac{\lambda(1-\beta)}{\beta \gamma+\lambda} \eta_{1} S_{1} V_{1} \frac{S V L_{1}}{S_{1} V_{1} L} \\
& +\frac{\lambda(1-\beta)}{\beta \gamma+\lambda} \eta_{1} S_{1} V_{1}-\frac{\beta(\gamma+\lambda)}{\beta \gamma+\lambda} \eta_{1} S_{1} V_{1} \frac{S V I_{1}}{S_{1} V_{1} I} \\
& -\frac{\lambda(1-\beta)}{\beta \gamma+\lambda} \eta_{1} S_{1} V_{1} \frac{L I_{1}}{L_{1} I}+\eta_{1} S_{1} V_{1} \\
& +\frac{\mu_{1}(\gamma+\lambda)}{\beta \gamma+\lambda} C^{I} I_{1}+\frac{r}{\varphi} Y-\frac{\delta(\psi+\omega)}{\varphi \psi} Y-\eta_{1} S_{1} V_{1} \frac{I V_{1}}{I_{1} V} \\
& +\eta_{1} S_{1} V_{1}-\frac{\mu_{1} \pi_{1}(\gamma+\lambda)}{\sigma_{1}(\beta \gamma+\lambda)} C^{I} \\
& -\frac{\mu_{2} \pi_{2}(\psi+\omega)}{\varphi \psi \sigma_{2}} C^{Y} \\
& =-\alpha \frac{\left(S-S_{1}\right)^{2}}{S}+\frac{\lambda(1-\beta)}{\beta \gamma+\lambda} \eta_{1} S_{1} V_{1}\left(4-\frac{S_{1}}{S}-\frac{S V L_{1}}{S_{1} V_{1} L}-\frac{L I_{1}}{L_{1} I}-\frac{I V_{1}}{I_{1} V}\right) \\
& +\frac{\beta(\gamma+\lambda)}{\beta \gamma+\lambda} \eta_{1} S_{1} V_{1}\left(3-\frac{S_{1}}{S}-\frac{S V I_{1}}{S_{1} V_{1} I}-\frac{I V_{1}}{I_{1} V}\right) \\
& +\frac{(\delta-r) \psi+\delta \omega}{\varphi \psi}\left(\frac{\eta_{2} \varphi \psi S_{1}}{(\delta-r) \psi+\delta \omega}-1\right) Y \\
& +\frac{\mu_{1} \pi_{1}(\gamma+\lambda)}{\sigma_{1}(\beta \gamma+\lambda)}\left(\frac{\sigma_{1} I_{1}}{\pi_{1}}-1\right) C^{I}-\frac{\mu_{2} \pi_{2}(\psi+\omega)}{\varphi \psi \sigma_{2}} C^{Y} .
\end{aligned}
$$

Therefore Eq. (16) becomes

$$
\begin{aligned}
\frac{\mathrm{d} \Phi_{1}}{\mathrm{~d} t}= & -\alpha \frac{\left(S-S_{1}\right)^{2}}{S}+\frac{\lambda(1-\beta)}{\beta \gamma+\lambda} \eta_{1} S_{1} V_{1}\left(4-\frac{S_{1}}{S}-\frac{S V L_{1}}{S_{1} V_{1} L}-\frac{L I_{1}}{L_{1} I}-\frac{I V_{1}}{I_{1} V}\right) \\
& +\frac{\beta(\gamma+\lambda)}{\beta \gamma+\lambda} \eta_{1} S_{1} V_{1}\left(3-\frac{S_{1}}{S}-\frac{S V I_{1}}{S_{1} V_{1} I}-\frac{I V_{1}}{I_{1} V}\right) \\
& +\frac{(\delta-r) \psi+\delta \omega}{\varphi \psi}\left(\mathfrak{R}_{2} / \mathfrak{R}_{1}-1\right) Y \\
& +\frac{\mu_{1}(\gamma+\lambda)\left(\varepsilon \alpha \sigma_{1}+\pi_{1} \eta_{1} b\right)}{\sigma_{1} \eta_{1} b(\beta \gamma+\lambda)}\left(\mathfrak{R}_{3}-1\right) C^{I}-\frac{\mu_{2} \pi_{2}(\psi+\omega)}{\varphi \psi \sigma_{2}} C^{Y} .
\end{aligned}
$$

Since $\mathfrak{R}_{2} / \mathfrak{R}_{1} \leq 1$ and $\mathfrak{R}_{3} \leq 1$, then using inequalities (13)-(14) we get $\frac{\mathrm{d} \Phi_{1}}{\mathrm{~d} t} \leq 0$ for all $S, L, I, Y, V, C^{I}, C^{Y}>0$ with equality holding when $S=S_{1}, L=L_{1}, I=I_{1}, V=$ $V_{1}$ and $Y=C^{I}=C^{Y}=0$. The trajectories of system (4) converge to $\Upsilon_{1}^{\prime}$ which includes elements with $Y=0$ and then $\dot{Y}=0$. The fifth equation of system (4) implies 


$$
0=\dot{Y}=\psi E
$$

which yields $E(t)=0$ for all $t$. Hence, $\Upsilon_{1}^{\prime}=\left\{\bigoplus_{1}\right\}$ and $\bigoplus_{1}$ is G.A.S using Lyapunov-LaSalle asymptotic stability theorem.

Theorem 3 If $\mathfrak{R}_{2}>1, \mathfrak{R}_{1} / \mathfrak{R}_{2} \leq 1$ and $\mathfrak{R}_{4} \leq 1$, then $\bigoplus_{2}$ is G.A.S.

Proof Define $\Phi_{2}\left(S, L, I, E, Y, V, C^{I}, C^{Y}\right)$ as:

$$
\begin{aligned}
\Phi_{2}= & S_{2} F\left(\frac{S}{S_{2}}\right)+\frac{\lambda}{\beta \gamma+\lambda} L+\frac{\gamma+\lambda}{\beta \gamma+\lambda} I+\frac{1}{\varphi} E_{2} F\left(\frac{E}{E_{2}}\right)+\frac{\psi+\omega}{\varphi \psi} Y_{2} F\left(\frac{Y}{Y_{2}}\right) \\
& +\frac{a(\gamma+\lambda)}{b(\beta \gamma+\lambda)} V+\frac{\mu_{1}(\gamma+\lambda)}{\sigma_{1}(\beta \gamma+\lambda)} C^{I}+\frac{\mu_{2}(\psi+\omega)}{\varphi \psi \sigma_{2}} C^{Y} .
\end{aligned}
$$

We calculate $\frac{\mathrm{d} \Phi_{2}}{\mathrm{~d} t}$ as:

$$
\begin{aligned}
\frac{\mathrm{d} \Phi_{2}}{\mathrm{~d} t}= & \left(1-\frac{S_{2}}{S}\right)\left[\rho-\alpha S-\eta_{1} S V-\eta_{2} S Y\right] \\
& +\frac{\lambda}{\beta \gamma+\lambda}\left[(1-\beta) \eta_{1} S V-(\lambda+\gamma) L\right] \\
& +\frac{\gamma+\lambda}{\beta \gamma+\lambda}\left[\beta \eta_{1} S V+\lambda L-a I-\mu_{1} C^{I} I\right] \\
& +\frac{1}{\varphi}\left(1-\frac{E_{2}}{E}\right)\left[\varphi \eta_{2} S Y+r Y-(\psi+\omega) E\right] \\
& +\frac{\psi+\omega}{\varphi \psi}\left(1-\frac{Y_{2}}{Y}\right)\left[\psi E-\delta Y-\mu_{2} C^{Y} Y\right]+\frac{a(\gamma+\lambda)}{b(\beta \gamma+\lambda)}[b I-\varepsilon V] \\
& +\frac{\mu_{1}(\gamma+\lambda)}{\sigma_{1}(\beta \gamma+\lambda)}\left[\sigma_{1} C^{I} I-\pi_{1} C^{I}\right]+\frac{\mu_{2}(\psi+\omega)}{\varphi \psi \sigma_{2}}\left[\sigma_{2} C^{Y} Y-\pi_{2} C^{Y}\right] \\
= & \left(1-\frac{S_{2}}{S}\right)(\rho-\alpha S)+\eta_{1} S_{2} V+\eta_{2} S_{2} Y+\frac{r}{\varphi} Y \\
& -\eta_{2} S Y \frac{E_{2}}{E}-\frac{r}{\varphi} Y \frac{E_{2}}{E}+\frac{\psi+\omega}{\varphi} E_{2} \\
& -\frac{\delta(\psi+\omega)}{\varphi \psi} Y-\frac{\psi+\omega}{\varphi} E_{\frac{Y_{2}}{Y}}+\frac{\delta(\psi+\omega)}{\varphi \psi} Y_{2}+\frac{\mu_{2}(\psi+\omega)}{\varphi \psi} C^{Y} Y_{2} \\
& -\frac{a \varepsilon(\gamma+\lambda)}{b(\beta \gamma+\lambda)} V-\frac{\mu_{1} \pi_{1}(\gamma+\lambda)}{\sigma_{1}(\beta \gamma+\lambda)} C^{I}-\frac{\mu_{2} \pi_{2}(\psi+\omega)}{\varphi \psi \sigma_{2}} C^{Y} .
\end{aligned}
$$

Using the equilibrium conditions for $\mathrm{Ð}_{2}$ :

$$
\rho=\alpha S_{2}+\eta_{2} S_{2} Y_{2}, \quad \eta_{2} S_{2} Y_{2}+\frac{r}{\varphi} Y_{2}=\frac{\psi+\omega}{\varphi} E_{2}=\frac{\delta(\psi+\omega)}{\varphi \psi} Y_{2},
$$

we obtain 


$$
\begin{aligned}
& \frac{\mathrm{d} \Phi_{2}}{\mathrm{~d} t}=\left(1-\frac{S_{2}}{S}\right)\left(\alpha S_{2}-\alpha S\right)+\eta_{2} S_{2} Y_{2}\left(1-\frac{S_{2}}{S}\right) \\
& +\eta_{1} S_{2} V-\eta_{2} S_{2} Y_{2} \frac{S Y E_{2}}{S_{2} Y_{2} E}-\frac{r}{\varphi} Y_{2} \frac{Y E_{2}}{Y_{2} E} \\
& +\eta_{2} S_{2} Y_{2}+\frac{r}{\varphi} Y_{2}-\eta_{2} S_{2} Y_{2} \frac{E Y_{2}}{E_{2} Y}-\frac{r}{\varphi} Y_{2} \frac{E Y_{2}}{E_{2} Y} \\
& +\eta_{2} S_{2} Y_{2}+\frac{r}{\varphi} Y_{2}+\frac{\mu_{2}(\psi+\omega)}{\varphi \psi} C^{Y} Y_{2} \\
& -\frac{a \varepsilon(\gamma+\lambda)}{b(\beta \gamma+\lambda)} V-\frac{\mu_{1} \pi_{1}(\gamma+\lambda)}{\sigma_{1}(\beta \gamma+\lambda)} C^{I}-\frac{\mu_{2} \pi_{2}(\psi+\omega)}{\varphi \psi \sigma_{2}} C^{Y} \\
& =-\alpha \frac{\left(S-S_{2}\right)^{2}}{S}+\eta_{2} S_{2} Y_{2}\left(3-\frac{S_{2}}{S}-\frac{S Y E_{2}}{S_{2} Y_{2} E}-\frac{E Y_{2}}{E_{2} Y}\right) \\
& +\frac{r}{\varphi} Y_{2}\left(2-\frac{Y E_{2}}{Y_{2} E}-\frac{E Y_{2}}{E_{2} Y}\right) \\
& +\frac{a \varepsilon(\gamma+\lambda)}{b(\beta \gamma+\lambda)}\left(\frac{\eta_{1} S_{2} b(\beta \gamma+\lambda)}{a \varepsilon(\gamma+\lambda)}-1\right) V-\frac{\mu_{1} \pi_{1}(\gamma+\lambda)}{\sigma_{1}(\beta \gamma+\lambda)} C^{I} \\
& +\frac{\mu_{2} \pi_{2}(\psi+\omega)}{\varphi \psi \sigma_{2}}\left(\frac{\sigma_{2} Y_{2}}{\pi_{2}}-1\right) C^{Y} \\
& =-\alpha \frac{\left(S-S_{2}\right)^{2}}{S}-\frac{r}{\varphi} \frac{\left(Y E_{2}-E Y_{2}\right)^{2}}{E E_{2} Y}+\eta_{2} S_{2} Y_{2}\left(3-\frac{S_{2}}{S}-\frac{S Y E_{2}}{S_{2} Y_{2} E}-\frac{E Y_{2}}{E_{2} Y}\right) \\
& +\frac{a \varepsilon(\gamma+\lambda)}{b(\beta \gamma+\lambda)}\left(\mathfrak{R}_{1} / \mathfrak{R}_{2}-1\right) V-\frac{\mu_{1} \pi_{1}(\gamma+\lambda)}{\sigma_{1}(\beta \gamma+\lambda)} C^{I} \\
& +\frac{\mu_{2}(\psi+\omega)\left(\alpha \sigma_{2}+\eta_{2} \pi_{2}\right)}{\varphi \psi \eta_{2} \sigma_{2}}\left(\mathfrak{R}_{4}-1\right) C^{Y} \text {. }
\end{aligned}
$$

Thus, if $\mathfrak{R}_{1} / \mathfrak{R}_{2} \leq 1$ and $\mathfrak{R}_{4} \leq 1$, then using inequality (15) we get $\frac{\mathrm{d} \Phi_{2}}{\mathrm{~d} t} \leq 0$ for all $S, L, I, E, Y, V, C^{I}, C^{Y}>0$. Moreover, $\frac{\mathrm{d} \Phi_{2}}{\mathrm{~d} t}=0$ at $S=S_{2}, E=E_{2}, Y=Y_{2}$ and $V=$ $C^{I}=C^{Y}=0$. The solutions of system (4) converge to $\Upsilon_{2}^{\prime}$ which contains elements with $V=0$, and then $\dot{V}=0$. The sixth equation of system (4) implies

$$
0=\dot{V}=b I \text {. }
$$

Then we get $I(t)=0$ for all $t$. Moreover, we have $\dot{I}=0$. Thus, the third equation of system (4) gives

$$
0=\dot{I}=\lambda L
$$

which yields $L(t)=0$ for all $t$. Therefore, $\Upsilon_{2}^{\prime}=\left\{\bigoplus_{2}\right\}$. By applying LyapunovLaSalle asymptotic stability theorem we get that $\bigoplus_{2}$ is G.A.S. 
Theorem 4 If $\mathfrak{R}_{3}>1$ and $\mathfrak{R}_{5} \leq 1$, then $\bigoplus_{3}$ is G.A.S.

Proof Define a function $\Phi_{3}\left(S, L, I, E, Y, V, C^{I}, C^{Y}\right)$ as:

$$
\begin{aligned}
\Phi_{3}= & S_{3} F\left(\frac{S}{S_{3}}\right)+\frac{\lambda}{\beta \gamma+\lambda} L_{3} F\left(\frac{L}{L_{3}}\right)+\frac{\gamma+\lambda}{\beta \gamma+\lambda} I_{3} F\left(\frac{I}{I_{3}}\right)+\frac{1}{\varphi} E+\frac{\psi+\omega}{\varphi \psi} Y \\
& +\frac{\eta_{1} S_{3}}{\varepsilon} V_{3} F\left(\frac{V}{V_{3}}\right)+\frac{\mu_{1}(\gamma+\lambda)}{\sigma_{1}(\beta \gamma+\lambda)} C_{3}^{I} F\left(\frac{C^{I}}{C_{3}^{I}}\right)+\frac{\mu_{2}(\psi+\omega)}{\varphi \psi \sigma_{2}} C^{Y} .
\end{aligned}
$$

We calculate $\frac{\mathrm{d} \Phi_{3}}{\mathrm{~d} t}$ as:

$$
\begin{aligned}
& \frac{\mathrm{d} \Phi_{3}}{\mathrm{~d} t}=\left(1-\frac{S_{3}}{S}\right)\left[\rho-\alpha S-\eta_{1} S V-\eta_{2} S Y\right]+\frac{\lambda}{\beta \gamma+\lambda}\left(1-\frac{L_{3}}{L}\right) \\
& \times\left[(1-\beta) \eta_{1} S V-(\lambda+\gamma) L\right] \\
& +\frac{\gamma+\lambda}{\beta \gamma+\lambda}\left(1-\frac{I_{3}}{I}\right)\left[\beta \eta_{1} S V+\lambda L-a I-\mu_{1} C^{I} I\right] \\
& +\frac{1}{\varphi}\left[\varphi \eta_{2} S Y+r Y-(\psi+\omega) E\right] \\
& +\frac{\psi+\omega}{\varphi \psi}\left[\psi E-\delta Y-\mu_{2} C^{Y} Y\right]+\frac{\eta_{1} S_{3}}{\varepsilon}\left(1-\frac{V_{3}}{V}\right)[b I-\varepsilon V] \\
& +\frac{\mu_{1}(\gamma+\lambda)}{\sigma_{1}(\beta \gamma+\lambda)}\left(1-\frac{C_{3}^{I}}{C^{I}}\right)\left[\sigma_{1} C^{I} I-\pi_{1} C^{I}\right] \\
& +\frac{\mu_{2}(\psi+\omega)}{\varphi \psi \sigma_{2}}\left[\sigma_{2} C^{Y} Y-\pi_{2} C^{Y}\right] \\
& =\left(1-\frac{S_{3}}{S}\right)(\rho-\alpha S)+\eta_{2} S_{3} Y-\frac{\lambda(1-\beta)}{\beta \gamma+\lambda} \eta_{1} S V \frac{L_{3}}{L}+\frac{\lambda(\gamma+\lambda)}{\beta \gamma+\lambda} L_{3} \\
& -\frac{a(\gamma+\lambda)}{\beta \gamma+\lambda} I-\frac{\beta(\gamma+\lambda)}{\beta \gamma+\lambda} \eta_{1} S V \frac{I_{3}}{I}-\frac{\lambda(\gamma+\lambda)}{\beta \gamma+\lambda} L \frac{I_{3}}{I}+\frac{a(\gamma+\lambda)}{\beta \gamma+\lambda} I_{3} \\
& +\frac{\mu_{1}(\gamma+\lambda)}{\beta \gamma+\lambda} C^{I} I_{3}+\frac{r}{\varphi} Y-\frac{\delta(\psi+\omega)}{\varphi \psi} Y+\frac{\eta_{1} S_{3}}{\varepsilon} b I \\
& -\frac{\eta_{1} S_{3}}{\varepsilon} b I \frac{V_{3}}{V}+\eta_{1} S_{3} V_{3} \\
& -\frac{\mu_{1} \pi_{1}(\gamma+\lambda)}{\sigma_{1}(\beta \gamma+\lambda)} C^{I}-\frac{\mu_{1}(\gamma+\lambda)}{\beta \gamma+\lambda} C_{3}^{I} I+\frac{\mu_{1} \pi_{1}(\gamma+\lambda)}{\sigma_{1}(\beta \gamma+\lambda)} C_{3}^{I} \\
& -\frac{\mu_{2} \pi_{2}(\psi+\omega)}{\varphi \psi \sigma_{2}} C^{Y}
\end{aligned}
$$

Using the equilibrium conditions for $\mathrm{Ð}_{3}$ : 


$$
\begin{aligned}
& \rho=\alpha S_{3}+\eta_{1} S_{3} V_{3}, \quad \frac{\lambda(1-\beta)}{\beta \gamma+\lambda} \eta_{1} S_{3} V_{3}=\frac{\lambda(\gamma+\lambda)}{\beta \gamma+\lambda} L_{3}, \\
& \eta_{1} S_{3} V_{3}=\frac{a(\gamma+\lambda)}{\beta \gamma+\lambda} I_{3}+\frac{\mu_{1}(\gamma+\lambda)}{\beta \gamma+\lambda} C_{3}^{I} I_{3}, \\
& I_{3}=\frac{\pi_{1}}{\sigma_{1}}, \quad V_{3}=\frac{b}{\varepsilon} I_{3}=\frac{b \pi_{1}}{\varepsilon \sigma_{1}},
\end{aligned}
$$

we obtain

$$
\begin{aligned}
\frac{\mathrm{d} \Phi_{3}}{\mathrm{~d} t}= & \left(1-\frac{S_{3}}{S}\right)\left(\alpha S_{3}-\alpha S\right)+\eta_{1} S_{3} V_{3}\left(1-\frac{S_{3}}{S}\right)+\left(\eta_{2} S_{3}-\frac{(\delta-r) \psi+\delta \omega}{\varphi \psi}\right) Y \\
& -\frac{\lambda(1-\beta)}{\beta \gamma+\lambda} \eta_{1} S_{3} V_{3} \frac{S V L_{3}}{S_{3} V_{3} L}+\frac{\lambda(1-\beta)}{\beta \gamma+\lambda} \eta_{1} S_{3} V_{3}-\frac{\beta(\gamma+\lambda)}{\beta \gamma+\lambda} \eta_{1} S_{3} V_{3} \frac{S V I_{3}}{S_{3} V_{3} I} \\
& -\frac{\lambda(1-\beta)}{\beta \gamma+\lambda} \eta_{1} S_{3} V_{3} \frac{L I_{3}}{L_{3} I}+\eta_{1} S_{3} V_{3}-\eta_{1} S_{3} V_{3} \frac{I V_{3}}{I_{3} V}+\eta_{1} S_{3} V_{3}-\frac{\mu_{2} \pi_{2}(\psi+\omega)}{\varphi \psi \sigma_{2}} C^{Y} \\
= & -\alpha \frac{\left(S-S_{3}\right)^{2}}{S}+\frac{\lambda(1-\beta)}{\beta \gamma+\lambda} \eta_{1} S_{3} V_{3}\left(4-\frac{S_{3}}{S}-\frac{S V L_{3}}{S_{3} V_{3} L}-\frac{L I_{3}}{L_{3} I}-\frac{I V_{3}}{I_{3} V}\right) \\
& +\frac{\beta(\gamma+\lambda)}{\beta \gamma+\lambda} \eta_{1} S_{3} V_{3}\left(3-\frac{S_{3}}{S}-\frac{S V I_{3}}{S_{3} V_{3} I}-\frac{I V_{3}}{I_{3} V}\right) \\
& +\frac{(\delta-r) \psi+\delta \omega}{\varphi \psi}\left(\frac{\varphi \psi \eta_{2} S_{3}}{(\delta-r) \psi+\delta \omega}-1\right) Y-\frac{\mu_{2} \pi_{2}(\psi+\omega)}{\varphi \psi \sigma_{2}} C^{Y} \\
= & -\alpha \frac{\left(S-S_{3}\right)^{2}}{S}+\frac{\lambda(1-\beta)}{\beta \gamma+\lambda} \eta_{1} S_{3} V_{3}\left(4-\frac{S_{3}}{S}-\frac{S V L_{3}}{S_{3} V_{3} L}-\frac{L I_{3}}{L_{3} I}-\frac{I V_{3}}{I_{3} V}\right) \\
& +\frac{\beta(\gamma+\lambda)}{\beta \gamma+\lambda} \eta_{1} S_{3} V_{3}\left(3-\frac{S_{3}}{S}-\frac{S V I_{3}}{S_{3} V_{3} I}-\frac{I V_{3}}{I_{3} V}\right)+\frac{(\delta-r) \psi+\delta \omega}{\varphi \psi}\left(\Re_{5}-1\right) Y \\
& -\frac{\mu_{2} \pi_{2}(\psi+\omega)}{\varphi \psi \sigma_{2}} C^{Y} .
\end{aligned}
$$

Hence, if $\mathfrak{R}_{5} \leq 1$, then using inequalities (13)-(14) we get $\frac{\mathrm{d} \Phi_{3}}{\mathrm{~d} t} \leq 0$ for all $S, L, I, E, Y, V, C^{I}, C^{Y}>0$. Moreover, $\frac{\mathrm{d} \Phi_{3}}{\mathrm{~d} t}=0$ at $S=S_{3}, L=L_{3}, I=I_{3}, V=V_{3}$ and $Y=C^{Y}=0$. We note that the solutions of system (4) tend to $\Upsilon_{3}^{\prime}$ which includes elements with $S(t)=S_{3}, L(t)=L_{3}, I(t)=I_{3}, V(t)=V_{3}, Y(t)=0$, then $\dot{I}(t)=0$, $\dot{Y}(t)=0$ and from the third and fifth equations of system (4) we have

$$
\begin{aligned}
& 0=\dot{I}(t)=\beta \eta_{1} S_{3} V_{3}+\lambda L_{3}-a I_{3}-\mu_{1} C^{I}(t) I_{3}, \\
& 0=\dot{Y}(t)=\psi E(t),
\end{aligned}
$$

which give $C^{I}(t)=C_{3}^{I}$ and $E(t)=0$ for all $t$. Therefore, $\Upsilon_{3}^{\prime}=\left\{\bigoplus_{3}\right\}$. Applying Lyapunov-LaSalle asymptotic stability theorem we get $\bigoplus_{3}$ is G.A.S.

Theorem 5 If $\mathfrak{R}_{4}>1$ and $\mathfrak{R}_{6} \leq 1$, then $\bigoplus_{4}$ is G.A.S.

Proof Define $\Phi_{4}\left(S, L, I, E, Y, V, C^{I}, C^{Y}\right)$ as: 


$$
\begin{aligned}
\Phi_{4}= & S_{4} F\left(\frac{S}{S_{4}}\right)+\frac{\lambda}{\beta \gamma+\lambda} L+\frac{\gamma+\lambda}{\beta \gamma+\lambda} I+\frac{1}{\varphi} E_{4} F\left(\frac{E}{E_{4}}\right)+\frac{\psi+\omega}{\varphi \psi} Y_{4} F\left(\frac{Y}{Y_{4}}\right) \\
& +\frac{a(\gamma+\lambda)}{b(\beta \gamma+\lambda)} V+\frac{\mu_{1}(\gamma+\lambda)}{\sigma_{1}(\beta \gamma+\lambda)} C^{I}+\frac{\mu_{2}(\psi+\omega)}{\varphi \psi \sigma_{2}} C_{4}^{Y} F\left(\frac{C^{Y}}{C_{4}^{Y}}\right) .
\end{aligned}
$$

Calculating $\frac{\mathrm{d} \Phi_{4}}{\mathrm{~d} t}$ as:

$$
\begin{aligned}
& \frac{\mathrm{d} \Phi_{4}}{\mathrm{~d} t}=\left(1-\frac{S_{4}}{S}\right)\left[\rho-\alpha S-\eta_{1} S V-\eta_{2} S Y\right] \\
& +\frac{\lambda}{\beta \gamma+\lambda}\left[(1-\beta) \eta_{1} S V-(\lambda+\gamma) L\right] \\
& +\frac{\gamma+\lambda}{\beta \gamma+\lambda}\left[\beta \eta_{1} S V+\lambda L-a I-\mu_{1} C^{I} I\right] \\
& +\frac{1}{\varphi}\left(1-\frac{E_{4}}{E}\right)\left[\varphi \eta_{2} S Y+r Y-(\psi+\omega) E\right] \\
& +\frac{\psi+\omega}{\varphi \psi}\left(1-\frac{Y_{4}}{Y}\right)\left[\psi E-\delta Y-\mu_{2} C^{Y} Y\right]+\frac{a(\gamma+\lambda)}{b(\beta \gamma+\lambda)}[b I-\varepsilon V] \\
& +\frac{\mu_{1}(\gamma+\lambda)}{\sigma_{1}(\beta \gamma+\lambda)}\left[\sigma_{1} C^{I} I-\pi_{1} C^{I}\right] \\
& +\frac{\mu_{2}(\psi+\omega)}{\varphi \psi \sigma_{2}}\left(1-\frac{C_{4}^{Y}}{C^{Y}}\right)\left[\sigma_{2} C^{Y} Y-\pi_{2} C^{Y}\right] \\
& =\left(1-\frac{S_{4}}{S}\right)(\rho-\alpha S)+\eta_{1} S_{4} V+\eta_{2} S_{4} Y+\frac{r}{\varphi} Y-\eta_{2} S Y \frac{E_{4}}{E} \\
& -\frac{r}{\varphi} Y \frac{E_{4}}{E}+\frac{\psi+\omega}{\varphi} E_{4} \\
& -\frac{\delta(\psi+\omega)}{\varphi \psi} Y-\frac{\psi+\omega}{\varphi} E \frac{Y_{4}}{Y}+\frac{\delta(\psi+\omega)}{\varphi \psi} Y_{4} \\
& +\frac{\mu_{2}(\psi+\omega)}{\varphi \psi} C^{Y} Y_{4}-\frac{a \varepsilon(\gamma+\lambda)}{b(\beta \gamma+\lambda)} V \\
& -\frac{\mu_{1} \pi_{1}(\gamma+\lambda)}{\sigma_{1}(\beta \gamma+\lambda)} C^{I}-\frac{\mu_{2} \pi_{2}(\psi+\omega)}{\varphi \psi \sigma_{2}} C^{Y} \\
& -\frac{\mu_{2}(\psi+\omega)}{\varphi \psi} C_{4}^{Y} Y+\frac{\mu_{2} \pi_{2}(\psi+\omega)}{\varphi \psi \sigma_{2}} C_{4}^{Y} \text {. }
\end{aligned}
$$

Using the equilibrium conditions for $\mathrm{Ð}_{4}$ :

$$
\begin{gathered}
\rho=\alpha S_{4}+\eta_{2} S_{4} Y_{4}, \quad Y_{4}=\frac{\pi_{2}}{\sigma_{2}} \\
\eta_{2} S_{4} Y_{4}+\frac{r}{\varphi} Y_{4}=\frac{\psi+\omega}{\varphi} E_{4}=\frac{\delta(\psi+\omega)}{\varphi \psi} Y_{4}+\frac{\mu_{2}(\psi+\omega)}{\varphi \psi} C_{4}^{Y} Y_{4}
\end{gathered}
$$

We obtain 


$$
\begin{aligned}
\frac{\mathrm{d} \Phi_{4}}{\mathrm{~d} t}= & \left(1-\frac{S_{4}}{S}\right)\left(\alpha S_{4}-\alpha S\right)+\eta_{2} S_{4} Y_{4}\left(1-\frac{S_{4}}{S}\right)+\eta_{1} S_{4} V-\eta_{2} S_{4} Y_{4} \frac{S Y E_{4}}{S_{4} Y_{4} E} \\
& -\frac{r}{\varphi} Y_{4} \frac{Y E_{4}}{Y_{4} E}+\eta_{2} S_{4} Y_{4}+\frac{r}{\varphi} Y_{4}-\eta_{2} S_{4} Y_{4} \frac{E Y_{4}}{E_{4} Y}-\frac{r}{\varphi} Y_{4} \frac{E Y_{4}}{E_{4} Y} \\
& +\eta_{2} S_{4} Y_{4}+\frac{r}{\varphi} Y_{4}-\frac{a \varepsilon(\gamma+\lambda)}{b(\beta \gamma+\lambda)} V-\frac{\mu_{1} \pi_{1}(\gamma+\lambda)}{\sigma_{1}(\beta \gamma+\lambda)} C^{I} \\
= & -\alpha \frac{\left(S-S_{4}\right)^{2}}{S}+\eta_{2} S_{4} Y_{4}\left(3-\frac{S_{4}}{S}-\frac{S Y E_{4}}{S_{4} Y_{4} E}-\frac{E Y_{4}}{E_{4} Y}\right) \\
& +\frac{r}{\varphi} Y_{4}\left(2-\frac{Y E_{4}}{Y_{4} E}-\frac{E Y_{4}}{E_{4} Y}\right) \\
& +\frac{a \varepsilon(\gamma+\lambda)}{b(\beta \gamma+\lambda)}\left(\frac{\eta_{1} b(\beta \gamma+\lambda) S_{4}}{a \varepsilon(\gamma+\lambda)}-1\right) V-\frac{\mu_{1} \pi_{1}(\gamma+\lambda)}{\sigma_{1}(\beta \gamma+\lambda)} C^{I} \\
= & -\alpha \frac{\left(S-S_{4}\right)^{2}}{S}-\frac{r}{\varphi} \frac{\left(Y E_{4}-E Y_{4}\right)^{2}}{E E_{4} Y}+\eta_{2} S_{4} Y_{4}\left(3-\frac{S_{4}}{S}-\frac{S Y E_{4}}{S_{4} Y_{4} E}-\frac{E Y_{4}}{E_{4} Y}\right) \\
& +\frac{a \varepsilon(\gamma+\lambda)}{b(\beta \gamma+\lambda)}\left(\Re_{6}-1\right) V-\frac{\mu_{1} \pi_{1}(\gamma+\lambda)}{\sigma_{1}(\beta \gamma+\lambda)} C^{I} .
\end{aligned}
$$

Hence, if $\mathfrak{R}_{6} \leq 1$, then using inequality (15) we get $\frac{\mathrm{d} \Phi_{4}}{\mathrm{~d} t} \leq 0$ for all $S, L, I, E, Y, V, C^{I}, C^{Y}>0$. In addition, we have $\frac{\mathrm{d} \Phi_{4}}{\mathrm{~d} t}=0$ at $S=S_{4}, E=E_{4}, Y=Y_{4}$ and $V=C^{I}=0$. The trajectories of system (4) converge to $\Upsilon_{4}^{\prime}$ which includes elements with $E=E_{4}, Y=Y_{4}, V=0$, and then $\dot{Y}=\dot{V}=0$. The fifth and sixth equations of system (4) imply

$$
\begin{aligned}
& 0=\dot{Y}=\psi E_{4}-\delta Y_{4}-\mu_{2} C^{Y} Y_{4}, \\
& 0=\dot{V}=b I,
\end{aligned}
$$

which give $C^{Y}(t)=C_{4}^{Y}$ and $I(t)=0$ for all $t$. Moreover, we have $I=0$, then from the third equation of system (4) we get

$$
0=\dot{I}=\lambda L,
$$

which yields $L(t)=0$ for all $t$. Therefore, $\Upsilon_{4}^{\prime}=\left\{\bigoplus_{4}\right\}$. By applying LyapunovLaSalle asymptotic stability theorem we get that $\bigoplus_{4}$ is G.A.S.

Theorem 6 If $\mathfrak{R}_{5}>1, \mathfrak{R}_{8} \leq 1$ and $\mathfrak{R}_{1} / \mathfrak{R}_{2}>1$, then $\emptyset_{5}$ is G.A.S.

Proof Define $\Phi_{5}\left(S, L, I, E, Y, V, C^{I}, C^{Y}\right)$ as:

$$
\begin{aligned}
\Phi_{5}= & S_{5} F\left(\frac{S}{S_{5}}\right)+\frac{\lambda}{\beta \gamma+\lambda} L_{5} F\left(\frac{L}{L_{5}}\right)+\frac{\gamma+\lambda}{\beta \gamma+\lambda} I_{5} F\left(\frac{I}{I_{5}}\right) \\
& +\frac{1}{\varphi} E_{5} F\left(\frac{E}{E_{5}}\right)+\frac{\psi+\omega}{\varphi \psi} Y_{5} F\left(\frac{Y}{Y_{5}}\right) \\
& +\frac{\eta_{1} S_{5}}{\varepsilon} V_{5} F\left(\frac{V}{V_{5}}\right)+\frac{\mu_{1}(\gamma+\lambda)}{\sigma_{1}(\beta \gamma+\lambda)} C_{5}^{I} F\left(\frac{C^{I}}{C_{5}^{I}}\right)+\frac{\mu_{2}(\psi+\omega)}{\varphi \psi \sigma_{2}} C^{Y} .
\end{aligned}
$$


Calculating $\frac{\mathrm{d} \Phi_{5}}{\mathrm{~d} t}$ as:

$$
\begin{aligned}
& \frac{\mathrm{d} \Phi_{5}}{\mathrm{~d} t}=\left(1-\frac{S_{5}}{S}\right)\left[\rho-\alpha S-\eta_{1} S V-\eta_{2} S Y\right]+\frac{\lambda}{\beta \gamma+\lambda}\left(1-\frac{L_{5}}{L}\right) \\
& \times\left[(1-\beta) \eta_{1} S V-(\lambda+\gamma) L\right] \\
& +\frac{\gamma+\lambda}{\beta \gamma+\lambda}\left(1-\frac{I_{5}}{I}\right)\left[\beta \eta_{1} S V+\lambda L-a I-\mu_{1} C^{I} I\right] \\
& +\frac{1}{\varphi}\left(1-\frac{E_{5}}{E}\right)\left[\varphi \eta_{2} S Y+r Y-(\psi+\omega) E\right] \\
& +\frac{\psi+\omega}{\varphi \psi}\left(1-\frac{Y_{5}}{Y}\right)\left[\psi E-\delta Y-\mu_{2} C^{Y} Y\right]+\frac{\eta_{1} S_{5}}{\varepsilon}\left(1-\frac{V_{5}}{V}\right)[b I-\varepsilon V] \\
& +\frac{\mu_{1}(\gamma+\lambda)}{\sigma_{1}(\beta \gamma+\lambda)}\left(1-\frac{C_{5}^{I}}{C^{I}}\right)\left[\sigma_{1} C^{I} I-\pi_{1} C^{I}\right] \\
& +\frac{\mu_{2}(\psi+\omega)}{\varphi \psi \sigma_{2}}\left[\sigma_{2} C^{Y} Y-\pi_{2} C^{Y}\right] \\
& =\left(1-\frac{S_{5}}{S}\right)(\rho-\alpha S)+\eta_{2} S_{5} Y-\frac{\lambda(1-\beta)}{\beta \gamma+\lambda} \eta_{1} S V \frac{L_{5}}{L} \\
& +\frac{\lambda(\gamma+\lambda)}{\beta \gamma+\lambda} L_{5}-\frac{a(\gamma+\lambda)}{\beta \gamma+\lambda} I \\
& -\frac{\beta(\gamma+\lambda)}{\beta \gamma+\lambda} \eta_{1} S V \frac{I_{5}}{I}-\frac{\lambda(\gamma+\lambda)}{\beta \gamma+\lambda} L \frac{I_{5}}{I} \\
& +\frac{a(\gamma+\lambda)}{\beta \gamma+\lambda} I_{5}+\frac{\mu_{1}(\gamma+\lambda)}{\beta \gamma+\lambda} C^{I} I_{5}+\frac{r}{\varphi} Y \\
& -\eta_{2} S Y \frac{E_{5}}{E}-\frac{r}{\varphi} Y \frac{E_{5}}{E}+\frac{\psi+\omega}{\varphi} E_{5}-\frac{\delta(\psi+\omega)}{\varphi \psi} Y \\
& -\frac{\psi+\omega}{\varphi} E \frac{Y_{5}}{Y}+\frac{\delta(\psi+\omega)}{\varphi \psi} Y_{5} \\
& +\frac{\mu_{2}(\psi+\omega)}{\varphi \psi} C^{Y} Y_{5}+\eta_{1} S_{5} \frac{b I}{\varepsilon}-\eta_{1} S_{5} V_{5} \frac{b I}{\varepsilon V} \\
& +\eta_{1} S_{5} V_{5}-\frac{\mu_{1} \pi_{1}(\gamma+\lambda)}{\sigma_{1}(\beta \gamma+\lambda)} C^{I} \\
& -\frac{\mu_{1}(\gamma+\lambda)}{\beta \gamma+\lambda} C_{5}^{I} I+\frac{\mu_{1} \pi_{1}(\gamma+\lambda)}{\sigma_{1}(\beta \gamma+\lambda)} C_{5}^{I}-\frac{\mu_{2} \pi_{2}(\psi+\omega)}{\varphi \psi \sigma_{2}} C^{Y} .
\end{aligned}
$$

Using the equilibrium conditions for $\bigoplus_{5}$ : 


$$
\begin{aligned}
& \rho=\alpha S_{5}+\eta_{1} S_{5} V_{5}+\eta_{2} S_{5} Y_{5}, \quad \frac{\lambda(1-\beta)}{\beta \gamma+\lambda} \eta_{1} S_{5} V_{5}=\frac{\lambda(\gamma+\lambda)}{\beta \gamma+\lambda} L_{5}, \\
& \eta_{1} S_{5} V_{5}=\frac{a(\gamma+\lambda)}{\beta \gamma+\lambda} I_{5}+\frac{\mu_{1}(\gamma+\lambda)}{\beta \gamma+\lambda} C_{5}^{I} I_{5}, \\
& \eta_{2} S_{5} Y_{5}+\frac{r}{\varphi} Y_{5}=\frac{\psi+\omega}{\varphi} E_{5}=\frac{\delta(\psi+\omega)}{\varphi \psi} Y_{5}, \\
& I_{5}=\frac{\pi_{1}}{\sigma_{1}}, \quad V_{5}=\frac{b I_{5}}{\varepsilon} .
\end{aligned}
$$

We obtain

$$
\begin{aligned}
& \frac{\mathrm{d} \Phi_{5}}{\mathrm{~d} t}=\left(1-\frac{S_{5}}{S}\right)\left(\alpha S_{5}-\alpha S\right)+\left(\eta_{1} S_{5} V_{5}+\eta_{2} S_{5} Y_{5}\right)\left(1-\frac{S_{5}}{S}\right) \\
& -\frac{\lambda(1-\beta)}{\beta \gamma+\lambda} \eta_{1} S_{5} V_{5} \frac{S V L_{5}}{S_{5} V_{5} L} \\
& +\frac{\lambda(1-\beta)}{\beta \gamma+\lambda} \eta_{1} S_{5} V_{5}-\frac{\beta(\gamma+\lambda)}{\beta \gamma+\lambda} \eta_{1} S_{5} V_{5} \frac{S V I_{5}}{S_{5} V_{5} I} \\
& -\frac{\lambda(1-\beta)}{\beta \gamma+\lambda} \eta_{1} S_{5} V_{5} \frac{L I_{5}}{L_{5} I}+\eta_{1} S_{5} V_{5} \\
& -\eta_{2} S_{5} Y_{5} \frac{S Y E_{5}}{S_{5} Y_{5} E}-\frac{r}{\varphi} Y_{5} \frac{Y E_{5}}{Y_{5} E}+\eta_{2} S_{5} Y_{5}+\frac{r}{\varphi} Y_{5} \\
& -\eta_{2} S_{5} Y_{5} \frac{E Y_{5}}{E_{5} Y}-\frac{r}{\varphi} Y_{5} \frac{E Y_{5}}{E_{5} Y} \\
& +\eta_{2} S_{5} Y_{5}+\frac{r}{\varphi} Y_{5}-\eta_{1} S_{5} V_{5} \frac{I V_{5}}{I_{5} V}+\eta_{1} S_{5} V_{5} \\
& +\frac{\mu_{2} \pi_{2}(\psi+\omega)}{\varphi \psi \sigma_{2}}\left(\frac{\sigma_{2} Y_{5}}{\pi_{2}}-1\right) C^{Y} \\
& =-\alpha \frac{\left(S-S_{5}\right)^{2}}{S}-\frac{r}{\varphi} \frac{\left(Y E_{5}-E Y_{5}\right)^{2}}{E E_{5} Y}+\frac{\lambda(1-\beta)}{\beta \gamma+\lambda} \eta_{1} S_{5} V_{5} \\
& \times\left(4-\frac{S_{5}}{S}-\frac{S V L_{5}}{S_{5} V_{5} L}-\frac{L I_{5}}{L_{5} I}-\frac{I V_{5}}{I_{5} V}\right) \\
& +\frac{\beta(\gamma+\lambda)}{\beta \gamma+\lambda} \eta_{1} S_{5} V_{5}\left(3-\frac{S_{5}}{S}-\frac{S V I_{5}}{S_{5} V_{5} I}-\frac{I V_{5}}{I_{5} V}\right) \\
& +\eta_{2} S_{5} Y_{5}\left(3-\frac{S_{5}}{S}-\frac{S Y E_{5}}{S_{5} Y_{5} E}-\frac{E Y_{5}}{E_{5} Y}\right) \\
& +\frac{\mu_{2} \pi_{2}(\psi+\omega)}{\varphi \psi \sigma_{2}}\left(\frac{\sigma_{2} Y_{5}}{\pi_{2}}-1\right) C^{Y} \text {. }
\end{aligned}
$$


Then, Eq. (20) will be reduced to the form

$$
\begin{aligned}
\frac{\mathrm{d} \Phi_{5}}{\mathrm{~d} t}= & -\alpha \frac{\left(S-S_{5}\right)^{2}}{S}-\frac{r}{\varphi} \frac{\left(Y E_{5}-E Y_{5}\right)^{2}}{E E_{5} Y}+\frac{\lambda(1-\beta)}{\beta \gamma+\lambda} \eta_{1} S_{5} V_{5} \\
& \times\left(4-\frac{S_{5}}{S}-\frac{S V L_{5}}{S_{5} V_{5} L}-\frac{L I_{5}}{L_{5} I}-\frac{I V_{5}}{I_{5} V}\right) \\
& +\frac{\beta(\gamma+\lambda)}{\beta \gamma+\lambda} \eta_{1} S_{5} V_{5}\left(3-\frac{S_{5}}{S}-\frac{S V I_{5}}{S_{5} V_{5} I}-\frac{I V_{5}}{I_{5} V}\right) \\
& +\eta_{2} S_{5} Y_{5}\left(3-\frac{S_{5}}{S}-\frac{S Y E_{5}}{S_{5} Y_{5} E}-\frac{E Y_{5}}{E_{5} Y}\right) \\
& +\frac{\mu_{2}(\psi+\omega)\left(\pi_{1} \eta_{1} b \sigma_{2}+\pi_{2} \eta_{2} \varepsilon \sigma_{1}+\alpha \varepsilon \sigma_{1} \sigma_{2}\right)}{\varphi \psi \eta_{2} \varepsilon \sigma_{1} \sigma_{2}}\left(\Re_{8}-1\right) C^{Y} .
\end{aligned}
$$

Hence, if $\mathfrak{R}_{8} \leq 1$, then using inequalities (13)-(15) we get $\frac{\mathrm{d} \Phi_{5}}{\mathrm{~d} t} \leq 0$ for all $S, L, I, E, Y, V, C^{I}, C^{Y}>0$. Further, $\frac{\mathrm{d} \Phi_{5}}{\mathrm{~d} t}=0$ when $S=S_{5}, L=L_{5}, I=I_{5}, E=E_{5}$, $Y=Y_{5}, V=V_{5}$ and $C^{Y}=0$. The solutions of system (4) converge to $\Upsilon_{5}^{\prime}$ which includes elements with $S=S_{5}, L=L_{5}, I=I_{5}, V=V_{5}$, and then $\dot{I}=0$. The third equation of system (4) implies

$$
0=\dot{I}=\beta \eta_{1} S_{5} V_{5}+\lambda L_{5}-a I_{5}-\mu_{1} C^{I} I_{5}
$$

which yields $C^{I}(t)=C_{5}^{I}$ for all $t$. Therefore, $\Upsilon_{5}^{\prime}=\left\{\bigoplus_{5}\right\}$. By applying LyapunovLaSalle asymptotic stability theorem we get $\bigoplus_{5}$ is G.A.S.

Theorem 7 If $\mathfrak{R}_{6}>1, \mathfrak{R}_{7} \leq 1$ and $\mathfrak{R}_{2} / \mathfrak{R}_{1}>1$, then $\bigoplus_{6}$ is G.A.S.

Proof Define $\Phi_{6}\left(S, L, I, E, Y, V, C^{I}, C^{Y}\right)$ as:

$$
\begin{aligned}
\Phi_{6}= & S_{6} F\left(\frac{S}{S_{6}}\right)+\frac{\lambda}{\beta \gamma+\lambda} L_{6} F\left(\frac{L}{L_{6}}\right)+\frac{\gamma+\lambda}{\beta \gamma+\lambda} I_{6} F\left(\frac{I}{I_{6}}\right) \\
& +\frac{1}{\varphi} E_{6} F\left(\frac{E}{E_{6}}\right)+\frac{\psi+\omega}{\varphi \psi} Y_{6} F\left(\frac{Y}{Y_{6}}\right) \\
& +\frac{\eta_{1} S_{6}}{\varepsilon} V_{6} F\left(\frac{V}{V_{6}}\right)+\frac{\mu_{1}(\gamma+\lambda)}{\sigma_{1}(\beta \gamma+\lambda)} C^{I}+\frac{\mu_{2}(\psi+\omega)}{\varphi \psi \sigma_{2}} C_{6}^{Y} F\left(\frac{C^{Y}}{C_{6}^{Y}}\right) .
\end{aligned}
$$

Calculating $\frac{\mathrm{d} \Phi_{6}}{\mathrm{~d} t}$ as: 


$$
\begin{aligned}
& \frac{\mathrm{d} \Phi_{6}}{\mathrm{~d} t}=\left(1-\frac{S_{6}}{S}\right)\left[\rho-\alpha S-\eta_{1} S V-\eta_{2} S Y\right] \\
& +\frac{\lambda}{\beta \gamma+\lambda}\left(1-\frac{L_{6}}{L}\right)\left[(1-\beta) \eta_{1} S V-(\lambda+\gamma) L\right] \\
& +\frac{\gamma+\lambda}{\beta \gamma+\lambda}\left(1-\frac{I_{6}}{I}\right)\left[\beta \eta_{1} S V+\lambda L-a I-\mu_{1} C^{I} I\right] \\
& +\frac{1}{\varphi}\left(1-\frac{E_{6}}{E}\right)\left[\varphi \eta_{2} S Y+r Y-(\psi+\omega) E\right] \\
& +\frac{\psi+\omega}{\varphi \psi}\left(1-\frac{Y_{6}}{Y}\right)\left[\psi E-\delta Y-\mu_{2} C^{Y} Y\right] \\
& +\frac{\eta_{1} S_{6}}{\varepsilon}\left(1-\frac{V_{6}}{V}\right)[b I-\varepsilon V] \\
& +\frac{\mu_{1}(\gamma+\lambda)}{\sigma_{1}(\beta \gamma+\lambda)}\left[\sigma_{1} C^{I} I-\pi_{1} C^{I}\right] \\
& +\frac{\mu_{2}(\psi+\omega)}{\varphi \psi \sigma_{2}}\left(1-\frac{C_{6}^{Y}}{C^{Y}}\right)\left[\sigma_{2} C^{Y} Y-\pi_{2} C^{Y}\right] \\
& =\left(1-\frac{S_{6}}{S}\right)(\rho-\alpha S)+\eta_{2} S_{6} Y-\frac{\lambda(1-\beta)}{\beta \gamma+\lambda} \eta_{1} S V \frac{L_{6}}{L} \\
& +\frac{\lambda(\gamma+\lambda)}{\beta \gamma+\lambda} L_{6}-\frac{a(\gamma+\lambda)}{\beta \gamma+\lambda} I \\
& -\frac{\beta(\gamma+\lambda)}{\beta \gamma+\lambda} \eta_{1} S V \frac{I_{6}}{I}-\frac{\lambda(\gamma+\lambda)}{\beta \gamma+\lambda} L \frac{I_{6}}{I}+\frac{a(\gamma+\lambda)}{\beta \gamma+\lambda} I_{6} \\
& +\frac{\mu_{1}(\gamma+\lambda)}{\beta \gamma+\lambda} C^{I} I_{6}+\frac{r}{\varphi} Y \\
& -\eta_{2} S Y \frac{E_{6}}{E}-\frac{r}{\varphi} Y \frac{E_{6}}{E}+\frac{\psi+\omega}{\varphi} E_{6}-\frac{\delta(\psi+\omega)}{\varphi \psi} Y \\
& -\frac{\psi+\omega}{\varphi} E \frac{Y_{6}}{Y}+\frac{\delta(\psi+\omega)}{\varphi \psi} Y_{6} \\
& +\frac{\mu_{2}(\psi+\omega)}{\varphi \psi} C^{Y} Y_{6}+\eta_{1} S_{6} \frac{b I}{\varepsilon}-\eta_{1} S_{6} V_{6} \frac{b I}{\varepsilon V}+\eta_{1} S_{6} V_{6} \\
& -\frac{\mu_{1} \pi_{1}(\gamma+\lambda)}{\sigma_{1}(\beta \gamma+\lambda)} C^{I} \\
& -\frac{\mu_{2} \pi_{2}(\psi+\omega)}{\varphi \psi \sigma_{2}} C^{Y}-\frac{\mu_{2}(\psi+\omega)}{\varphi \psi} C_{6}^{Y} Y+\frac{\mu_{2} \pi_{2}(\psi+\omega)}{\varphi \psi \sigma_{2}} C_{6}^{Y} .
\end{aligned}
$$

Using the equilibrium conditions for $\bigoplus_{6}$ : 


$$
\begin{aligned}
\rho & =\alpha S_{6}+\eta_{1} S_{6} V_{6}+\eta_{2} S_{6} Y_{6}, \quad \eta_{1} S_{6} V_{6}=\frac{a(\gamma+\lambda)}{\beta \gamma+\lambda} I_{6}, \\
\frac{\lambda(1-\beta)}{\beta \gamma+\lambda} \eta_{1} S_{6} V_{6} & =\frac{\lambda(\gamma+\lambda)}{\beta \gamma+\lambda} L_{6} \\
\eta_{2} S_{6} Y_{6}+\frac{r}{\varphi} Y_{6} & =\frac{\psi+\omega}{\varphi} E_{6}=\frac{\delta(\psi+\omega)}{\varphi \psi} Y_{6}+\frac{\mu_{2}(\psi+\omega)}{\varphi \psi} C_{6}^{Y} Y_{6}, \\
Y_{6} & =\frac{\pi_{2}}{\sigma_{2}}, \quad V_{6}=\frac{b I_{6}}{\varepsilon} .
\end{aligned}
$$

We obtain

$$
\begin{aligned}
\frac{\mathrm{d} \Phi_{6}}{\mathrm{~d} t}= & \left(1-\frac{S_{6}}{S}\right)\left(\alpha S_{6}-\alpha S\right)+\left(\eta_{1} S_{6} V_{6}+\eta_{2} S_{6} Y_{6}\right)\left(1-\frac{S_{6}}{S}\right) \\
& -\frac{\lambda(1-\beta)}{\beta \gamma+\lambda} \eta_{1} S_{6} V_{6} \frac{S V L_{6}}{S_{6} V_{6} L} \\
& +\frac{\lambda(1-\beta)}{\beta \gamma+\lambda} \eta_{1} S_{6} V_{6}-\frac{\beta(\gamma+\lambda)}{\beta \gamma+\lambda} \eta_{1} S_{6} V_{6} \frac{S V I_{6}}{S_{6} V_{6} I} \\
& -\frac{\lambda(1-\beta)}{\beta \gamma+\lambda} \eta_{1} S_{6} V_{6} \frac{L I_{6}}{L_{6} I}+\eta_{1} S_{6} V_{6} \\
& -\eta_{2} S_{6} Y_{6} \frac{S Y E_{6}}{S_{6} Y_{6} E}-\frac{r}{\varphi} Y_{6} \frac{Y E_{6}}{Y_{6} E}+\eta_{2} S_{6} Y_{6}+\frac{r}{\varphi} Y_{6}-\eta_{2} S_{6} Y_{6} \frac{E Y_{6}}{E_{6} Y}-\frac{r}{\varphi} Y_{6} \frac{E Y_{6}}{E_{6} Y} \\
& +\eta_{2} S_{6} Y_{6}+\frac{r}{\varphi} Y_{6}-\eta_{1} S_{6} V_{6} \frac{I V_{6}}{I_{6} V}+\eta_{1} S_{6} V_{6}+\frac{\mu_{1} \pi_{1}(\gamma+\lambda)}{\sigma_{1}(\beta \gamma+\lambda)}\left(\frac{\sigma_{1} I_{6}}{\pi_{1}}-1\right) C^{I} \\
= & -\alpha \frac{\left(S-S_{6}\right)^{2}}{S}+\frac{\lambda(1-\beta)}{\beta \gamma+\lambda} \eta_{1} S_{6} V_{6}\left(4-\frac{S_{6}}{S}-\frac{S V L_{6}}{S_{6} V_{6} L}-\frac{L I_{6}}{L_{6} I}-\frac{I V_{6}}{I_{6} V}\right) \\
& +\frac{\beta(\gamma+\lambda)}{\beta \gamma+\lambda} \eta_{1} S_{6} V_{6}\left(3-\frac{S_{6}}{S}-\frac{S V_{6}}{S_{6} V_{6} I}-\frac{I V_{6}}{I_{6} V}\right) \\
& +\eta_{2} S_{6} Y_{6}\left(3-\frac{S_{6}}{S}-\frac{S Y E_{6}}{S_{6} Y_{6} E}-\frac{E Y_{6}}{E_{6} Y}\right) \\
& +\frac{r}{\varphi} Y_{6}\left(2-\frac{Y E_{6}}{Y_{6} E}-\frac{E Y_{6}}{E_{6} Y}\right)+\frac{\mu_{1} \pi_{1}(\gamma+\lambda)}{\sigma_{1}(\beta \gamma+\lambda)}\left(\frac{\sigma_{1} I_{6}}{\pi_{1}}-1\right) C^{I} .
\end{aligned}
$$

Then, Eq. (21) will be reduced to the form

$$
\begin{aligned}
\frac{\mathrm{d} \Phi_{6}}{\mathrm{~d} t}= & -\alpha \frac{\left(S-S_{6}\right)^{2}}{S}-\frac{r}{\varphi} \frac{\left(Y E_{6}-E Y_{6}\right)^{2}}{E E_{6} Y}+\frac{\lambda(1-\beta)}{\beta \gamma+\lambda} \eta_{1} S_{6} V_{6} \\
& \times\left(4-\frac{S_{6}}{S}-\frac{S V L_{6}}{S_{6} V_{6} L}-\frac{L I_{6}}{L_{6} I}-\frac{I V_{6}}{I_{6} V}\right) \\
& +\frac{\beta(\gamma+\lambda)}{\beta \gamma+\lambda} \eta_{1} S_{6} V_{6}\left(3-\frac{S_{6}}{S}-\frac{S V I_{6}}{S_{6} V_{6} I}-\frac{I V_{6}}{I_{6} V}\right) \\
& +\eta_{2} S_{6} Y_{6}\left(3-\frac{S_{6}}{S}-\frac{S Y E_{6}}{S_{6} Y_{6} E}-\frac{E Y_{6}}{E_{6} Y}\right) \\
& +\frac{\mu_{1}(\gamma+\lambda)\left(\pi_{1} \eta_{1} b \sigma_{2}+\pi_{2} \eta_{2} \varepsilon \sigma_{1}+\alpha \varepsilon \sigma_{1} \sigma_{2}\right)}{\eta_{1} b \sigma_{1} \sigma_{2}(\beta \gamma+\lambda)}\left(\mathfrak{R}_{7}-1\right) C^{I} .
\end{aligned}
$$

Hence, if $\mathfrak{R}_{7} \leq 1$, then using inequalities (13)-(15) we get $\frac{\mathrm{d} \Phi_{6}}{\mathrm{~d} t} \leq 0$ for all 
$S, L, I, E, Y, V, C^{I}, C^{Y}>0$, where $\frac{\mathrm{d} \Phi_{6}}{\mathrm{~d} t}=0$ occurs at $S=S_{6}, L=L_{6}, I=I_{6}, E=E_{6}$, $Y=Y_{6}, V=V_{6}$ and $C^{I}=0$. The solutions of system (4) converge to $\Upsilon_{6}^{\prime}$ which contains elements with $E=E_{6}, Y=Y_{6}$, and then $\dot{Y}=0$. The fifth equation of system (4) implies

$$
0=\dot{Y}=\psi E_{6}-\delta Y_{6}-\mu_{2} C^{Y} Y_{6}
$$

which yields $C^{Y}(t)=C_{6}^{Y}$ for all $t$. Therefore, $\Upsilon_{6}^{\prime}=\left\{\bigoplus_{6}\right\}$ and then by applying Lyapunov-LaSalle asymptotic stability theorem we get that $\bigoplus_{6}$ is G.A.S.

Theorem 8 If $\mathfrak{R}_{7}>1$ and $\mathfrak{R}_{8}>1$, then $\emptyset_{7}$ is G.A.S.

Proof Define $\Phi_{7}\left(S, L, I, E, Y, V, C^{I}, C^{Y}\right)$ as:

$$
\begin{aligned}
\Phi_{7}= & S_{7} F\left(\frac{S}{S_{7}}\right)+\frac{\lambda}{\beta \gamma+\lambda} L_{7} F\left(\frac{L}{L_{7}}\right)+\frac{\gamma+\lambda}{\beta \gamma+\lambda} I_{7} F\left(\frac{I}{I_{7}}\right) \\
& +\frac{1}{\varphi} E_{7} F\left(\frac{E}{E_{7}}\right)+\frac{\psi+\omega}{\varphi \psi} Y_{7} F\left(\frac{Y}{Y_{7}}\right) \\
& +\frac{\eta_{1} S_{7}}{\varepsilon} V_{7} F\left(\frac{V}{V_{7}}\right)+\frac{\mu_{1}(\gamma+\lambda)}{\sigma_{1}(\beta \gamma+\lambda)} C_{7}^{I} F\left(\frac{C^{I}}{C_{7}^{I}}\right)+\frac{\mu_{2}(\psi+\omega)}{\varphi \psi \sigma_{2}} C_{7}^{Y} F\left(\frac{C^{Y}}{C_{7}^{Y}}\right) .
\end{aligned}
$$

Calculating $\frac{d \Phi_{7}}{d t}$ as:

$$
\begin{aligned}
\frac{\mathrm{d} \Phi_{7}}{\mathrm{~d} t}= & \left(1-\frac{S_{7}}{S}\right)\left[\rho-\alpha S-\eta_{1} S V-\eta_{2} S Y\right]+\frac{\lambda}{\beta \gamma+\lambda}\left(1-\frac{L_{7}}{L}\right) \\
& \times\left[(1-\beta) \eta_{1} S V-(\lambda+\gamma) L\right] \\
& +\frac{\gamma+\lambda}{\beta \gamma+\lambda}\left(1-\frac{I_{7}}{I}\right)\left[\beta \eta_{1} S V+\lambda L-a I-\mu_{1} C^{I} I\right] \\
& +\frac{1}{\varphi}\left(1-\frac{E_{7}}{E}\right)\left[\varphi \eta_{2} S Y+r Y-(\psi+\omega) E\right] \\
& +\frac{\psi+\omega}{\varphi \psi}\left(1-\frac{Y_{7}}{Y}\right)\left[\psi E-\delta Y-\mu_{2} C^{Y} Y\right]+\frac{\eta_{1} S_{7}}{\varepsilon}\left(1-\frac{V_{7}}{V}\right)[b I-\varepsilon V] \\
& +\frac{\mu_{1}(\gamma+\lambda)}{\sigma_{1}(\beta \gamma+\lambda)}\left(1-\frac{C_{7}^{I}}{C^{I}}\right)\left[\sigma_{1} C^{I} I-\pi_{1} C^{I}\right] \\
& +\frac{\mu_{2}(\psi+\omega)}{\varphi \psi \sigma_{2}}\left(1-\frac{C_{7}^{Y}}{C^{Y}}\right)\left[\sigma_{2} C^{Y} Y-\pi_{2} C^{Y}\right] \\
= & \left(1-\frac{S_{7}}{S}\right)(\rho-\alpha S)+\eta_{2} S_{7} Y-\frac{\lambda(1-\beta)}{\beta \gamma+\lambda} \eta_{1} S V \frac{L_{7}}{L}
\end{aligned}
$$




$$
\begin{aligned}
+ & \frac{\lambda(\gamma+\lambda)}{\beta \gamma+\lambda} L_{7}-\frac{a(\gamma+\lambda)}{\beta \gamma+\lambda} I \\
& -\frac{\beta(\gamma+\lambda)}{\beta \gamma+\lambda} \eta_{1} S V \frac{I_{7}}{I}-\frac{\lambda(\gamma+\lambda)}{\beta \gamma+\lambda} L \frac{I_{7}}{I}+\frac{a(\gamma+\lambda)}{\beta \gamma+\lambda} I_{7} \\
& +\frac{\mu_{1}(\gamma+\lambda)}{\beta \gamma+\lambda} C^{I} I_{7}+\frac{r}{\varphi} Y \\
& -\eta_{2} S Y \frac{E_{7}}{E}-\frac{r}{\varphi} Y \frac{E_{7}}{E}+\frac{\psi+\omega}{\varphi} E_{7}-\frac{\delta(\psi+\omega)}{\varphi \psi} Y \\
& -\frac{\psi+\omega}{\varphi} E \frac{Y_{7}}{Y}+\frac{\delta(\psi+\omega)}{\varphi \psi} Y_{7} \\
& +\frac{\mu_{2}(\psi+\omega)}{\varphi \psi} C^{Y} Y_{7}+\eta_{1} S_{7} \frac{b I}{\varepsilon}-\eta_{1} S_{7} V_{7} \frac{b I}{\varepsilon V}+\eta_{1} S_{7} V_{7} \\
- & \frac{\mu_{1} \pi_{1}(\gamma+\lambda)}{\sigma_{1}(\beta \gamma+\lambda)} C^{I} \\
- & \frac{\mu_{1}(\gamma+\lambda)}{\beta \gamma+\lambda} C_{7}^{I} I+\frac{\mu_{1} \pi_{1}(\gamma+\lambda)}{\sigma_{1}(\beta \gamma+\lambda)} C_{7}^{I}-\frac{\mu_{2} \pi_{2}(\psi+\omega)}{\varphi \psi \sigma_{2}} C^{Y} \\
- & \frac{\mu_{2}(\psi+\omega)}{\varphi \psi} C_{7}^{Y} Y+\frac{\mu_{2} \pi_{2}(\psi+\omega)}{\varphi \psi \sigma_{2}} C_{7}^{Y} .
\end{aligned}
$$

Using the equilibrium conditions for $\bigoplus_{7}$ :

$$
\begin{aligned}
& \rho=\alpha S_{7}+\eta_{1} S_{7} V_{7}+\eta_{2} S_{7} Y_{7}, \quad \frac{\lambda(1-\beta)}{\beta \gamma+\lambda} \eta_{1} S_{7} V_{7}=\frac{\lambda(\gamma+\lambda)}{\beta \gamma+\lambda} L_{7}, \\
& \eta_{1} S_{7} V_{7}=\frac{a(\gamma+\lambda)}{\beta \gamma+\lambda} I_{7}+\frac{\mu_{1}(\gamma+\lambda)}{\beta \gamma+\lambda} C_{7}^{I} I_{7}, \\
& \eta_{2} S_{7} Y_{7}+\frac{r}{\varphi} Y_{7}=\frac{\psi+\omega}{\varphi} E_{7}=\frac{\delta(\psi+\omega)}{\varphi \psi} Y_{7}+\frac{\mu_{2}(\psi+\omega)}{\varphi \psi} C_{7}^{Y} Y_{7}, \\
& I_{7}=\frac{\pi_{1}}{\sigma_{1}}, \quad Y_{7}=\frac{\pi_{2}}{\sigma_{2}}, \quad V_{7}=\frac{b I_{7}}{\varepsilon} .
\end{aligned}
$$

We obtain

$$
\begin{aligned}
\frac{\mathrm{d} \Phi_{7}}{\mathrm{~d} t}= & \left(1-\frac{S_{7}}{S}\right)\left(\alpha S_{7}-\alpha S\right)+\left(\eta_{1} S_{7} V_{7}+\eta_{2} S_{7} Y_{7}\right)\left(1-\frac{S_{7}}{S}\right) \\
& -\frac{\lambda(1-\beta)}{\beta \gamma+\lambda} \eta_{1} S_{7} V_{7} \frac{S V L_{7}}{S_{7} V_{7} L} \\
& +\frac{\lambda(1-\beta)}{\beta \gamma+\lambda} \eta_{1} S_{7} V_{7}-\frac{\beta(\gamma+\lambda)}{\beta \gamma+\lambda} \eta_{1} S_{7} V_{7} \frac{S V I_{7}}{S_{7} V_{7} I} \\
& -\frac{\lambda(1-\beta)}{\beta \gamma+\lambda} \eta_{1} S_{7} V_{7} \frac{L I_{7}}{L_{7} I}+\eta_{1} S_{7} V_{7}
\end{aligned}
$$




$$
\begin{aligned}
& -\eta_{2} S_{7} Y_{7} \frac{S Y E_{7}}{S_{7} Y_{7} E}-\frac{r}{\varphi} Y_{7} \frac{Y E_{7}}{Y_{7} E}+\eta_{2} S_{7} Y_{7}+\frac{r}{\varphi} Y_{7} \\
& -\eta_{2} S_{7} Y_{7} \frac{E Y_{7}}{E_{7} Y}-\frac{r}{\varphi} Y_{7} \frac{E Y_{7}}{E_{7} Y} \\
& +\eta_{2} S_{7} Y_{7}+\frac{r}{\varphi} Y_{7}-\eta_{1} S_{7} V_{7} \frac{I V_{7}}{I_{7} V}+\eta_{1} S_{7} V_{7} \\
& =-\alpha \frac{\left(S-S_{7}\right)^{2}}{S}-\frac{r}{\varphi} \frac{\left(Y E_{7}-E Y_{7}\right)^{2}}{E E_{7} Y}+\frac{\lambda(1-\beta)}{\beta \gamma+\lambda} \eta_{1} S_{7} V_{7} \\
& \times\left(4-\frac{S_{7}}{S}-\frac{S V L_{7}}{S_{7} V_{7} L}-\frac{L I_{7}}{L_{7} I}-\frac{I V_{7}}{I_{7} V}\right) \\
& +\frac{\beta(\gamma+\lambda)}{\beta \gamma+\lambda} \eta_{1} S_{7} V_{7}\left(3-\frac{S_{7}}{S}-\frac{S V I_{7}}{S_{7} V_{7} I}-\frac{I V_{7}}{I_{7} V}\right) \\
& +\eta_{2} S_{7} Y_{7}\left(3-\frac{S_{7}}{S}-\frac{S Y E_{7}}{S_{7} Y_{7} E}-\frac{E Y_{7}}{E_{7} Y}\right) .
\end{aligned}
$$

Then using inequalities (13)-(15) we get $\frac{\mathrm{d} \Phi_{7}}{\mathrm{~d} t} \leq 0$ for all $S, L, I, E, Y, V, C^{I}, C^{Y}>0$. Further, $\frac{\mathrm{d} \Phi_{7}}{\mathrm{~d} t}=0$ when $S=S_{7}, L=L_{7}, I=I_{7}, E=E_{7}, Y=Y_{7}$ and $V=V_{7}$. The solutions of system (4) lead to $\Upsilon_{7}^{\prime}$ which includes elements with $S=S_{7}, L=L_{7}$, $I=I_{7}, E=E_{7}, Y=Y_{7}$ and $V=V_{7}$, and then $\dot{I}=\dot{Y}=0$. The third and fifth equations of system (4) imply

$$
\begin{aligned}
& 0=\dot{I}=\beta \eta_{1} S_{7} V_{7}+\lambda L_{7}-a I_{7}-\mu_{1} C^{I} I_{7}, \\
& 0=\dot{Y}=\psi E_{7}-\delta Y_{7}-\mu_{2} C^{Y} Y_{7},
\end{aligned}
$$

which ensure that $C^{I}(t)=C_{7}^{I}$ and $C^{Y}(t)=C_{7}^{Y}$ for all $t$. Thus $\Upsilon_{7}^{\prime}=\left\{\bigoplus_{7}\right\}$ and by applying Lyapunov-LaSalle asymptotic stability theorem we get that $\bigoplus_{7}$ is G.A.S.

In Table 3, we summarize the global stability results given in Theorems 1-8.

\section{Numerical simulations}

In this section, we illustrate the results of Theorems $1-8$ by performing numerical simulations. Moreover, we study the effect of HTLV-I infection on the HIV monoinfected individuals by making a comparison between the dynamics of HIV monoinfection and HIV/HTLV-I co-infection. Otherwise, we investigate the influence of HIV infection on the HTLV-I mono-infected individuals by conducting a comparison between the dynamics of HTLV-I mono-infection and HIV/HTLV-I co-infection. 
For solving system (3) numerically we fix the values of some parameters taken from literature as mentioned in Table 4 . To verify the stability of the eight equilibria given in Theorems 1-8, we vary some parameters that affect the values of the threshold parameters which in turn control the existence and stability of the equilibria. We confirm that we have assumed some values of the model's parameters just to conduct the numerical simulations. In fact, it is challenging to collect real data from HIV/HTLV-I co-infected patients. However, if one has real data then the model's parameters can be estimated and the validity of the model can be established.

\subsection{Stability of the equilibria}

In this subsection, we illustrate our global stability results given in Theorems 1-8. To do so, we show that the solution of the system starting from any initial point (at any disease stage) in the feasible set $\Theta$ will converge to only one of the eight equilibria of the system. Therefore, we choose three different initial conditions for the system (3) as follows:

\section{Initial-1 :}

$\left(S(0), L(0), I(0), E(0), Y(0), V(0), C^{I}(0), C^{Y}(0)\right)=(600,1.5,1.5,30,0.3,5,1,3)$,

\section{Initial-2:}

$\left(S(0), L(0), I(0), E(0), Y(0), V(0), C^{I}(0), C^{Y}(0)\right)=(500,1,1,20,0.2,2,2,2)$,

Initial-3:

$\left(S(0), L(0), I(0), E(0), Y(0), V(0), C^{I}(0), C^{Y}(0)\right)=(300,0.5,0.5,10,0.1,1.5,3,1)$.

Choosing selected values of $\eta_{1}, \eta_{2}, \sigma_{1}$ and $\sigma_{2}$ under the above initial conditions leads to the following scenarios:

Scenario 1 (Stability of $\boldsymbol{\oplus}_{0}$ ): $\eta_{1}=0.0001, \eta_{2}=0.001$ and $\sigma_{1}=\sigma_{2}=0.2$. For this set of parameters, we have $\mathfrak{R}_{1}=0.49<1$ and $\mathfrak{R}_{2}=0.23<1$. Figure 2 displays that the trajectories initiating with Initial-1, Initial-2 and Initial-3 reach the

Table 3 Global stability conditions of the equilibria of model (4)

\begin{tabular}{ll}
\hline Equilibrium point & Global stability conditions \\
\hline$\bigoplus_{0}=\left(S_{0}, 0,0,0,0,0,0,0\right)$ & $\mathfrak{R}_{1} \leq 1$ and $\mathfrak{R}_{2} \leq 1$ \\
$\bigoplus_{1}=\left(S_{1}, L_{1}, I_{1}, 0,0, V_{1}, 0,0\right)$ & $\mathfrak{R}_{1}>1, \mathfrak{R}_{2} / \mathfrak{R}_{1} \leq 1$ and $\mathfrak{R}_{3} \leq 1$ \\
$\bigoplus_{2}=\left(S_{2}, 0,0, E_{2}, Y_{2}, 0,0,0\right)$ & $\mathfrak{R}_{2}>1, \mathfrak{R}_{1} / \mathfrak{R}_{2} \leq 1$ and $\mathfrak{R}_{4} \leq 1$ \\
$\bigoplus_{3}=\left(S_{3}, L_{3}, I_{3}, 0,0, V_{3}, C_{3}^{I}, 0\right)$ & $\mathfrak{R}_{3}>1$ and $\mathfrak{R}_{5} \leq 1$ \\
$\bigoplus_{4}=\left(S_{4}, 0,0, E_{4}, Y_{4}, 0,0, C_{4}^{Y}\right)$ & $\mathfrak{R}_{4}>1$ and $\mathfrak{R}_{6} \leq 1$ \\
$\bigoplus_{5}=\left(S_{5}, L_{5}, I_{5}, E_{5}, Y_{5}, V_{5}, C_{5}^{I}, 0\right)$ & $\mathfrak{R}_{5}>1, \mathfrak{R}_{8} \leq 1$ and $\mathfrak{R}_{1} / \mathfrak{R}_{2}>1$ \\
$\bigoplus_{6}=\left(S_{6}, L_{6}, I_{6}, E_{6}, Y_{6}, V_{6}, 0, C_{6}^{Y}\right)$ & $\mathfrak{R}_{6}>1, \mathfrak{R}_{7} \leq 1$ and $\mathfrak{R}_{2} / \mathfrak{R}_{1}>1$ \\
$\bigoplus_{7}=\left(S_{7}, L_{7}, I_{7}, E_{7}, Y_{7}, V_{7}, C_{7}^{I}, C_{7}^{Y}\right)$ & $\mathfrak{R}_{7}>1$ and $\mathfrak{R}_{8}>1$ \\
\hline
\end{tabular}


Table 4 The values and sources of parameters of model (3)

\begin{tabular}{lllllllll}
\hline Parameter & Value & Source & Parameter & Value & Source & Parameter & Value & Source \\
\hline$\rho$ & 10 & {$[35,57]$} & $\pi_{1}$ & 0.1 & {$[59]$} & $\gamma$ & 0.02 & Assumed \\
$\alpha$ & 0.01 & {$[8,58]$} & $\pi_{2}$ & 0.1 & Assumed & $\psi$ & 0.003 & {$[35]$} \\
$a$ & 0.5 & {$[7]$} & $r^{*}$ & 0.007 & Assumed & $\lambda$ & 0.2 & {$[60]$} \\
$\kappa$ & 0.3 & {$[24]$} & $\mu_{1}$ & 0.2 & Assumed & $\omega$ & 0.01 & {$[35]$} \\
$\varphi$ & 0.2 & {$[24]$} & $\mu_{2}$ & 0.2 & {$[37]$} & & & \\
$\delta^{*}$ & 0.2 & {$[37]$} & $\varepsilon$ & 2 & Assumed & & & \\
$b$ & 5 & Assumed & $\beta$ & 0.7 & {$[5]$} & & & \\
\hline
\end{tabular}

equilibrium $\mathrm{Ð}_{0}=(1000,0,0,0,0,0,0,0)$. This shows that $\mathrm{Ð}_{0}$ is G.A.S according to Theorem 1. In this situation both HIV and HTLV-I will be died out.

Scenario 2 (Stability of $\boldsymbol{D}_{1}$ ): $\eta_{1}=\eta_{2}=0.0005, \sigma_{1}=0.003$ and $\sigma_{2}=0.2$. With such choice we get $\mathfrak{R}_{2}=0.12<1<2.43=\mathfrak{R}_{1}, \mathfrak{R}_{3}=0.47<1$ and hence $\mathfrak{R}_{2} / \mathfrak{R}_{1}=0.05<1$. Therefore, the conditions in Table 2 are verified. In fact, the equilibrium point $\mathrm{Ð}_{1}$ exists with $\mathrm{Ð}_{1}=(411.22,8.03,11.45,0,0,28.64,0,0)$. Figure 3 displays that the trajectories initiating with Initial-1, Initial-2 and Initial-3 tend to $\bigoplus_{1}$. Therefore, the numerical results support Theorem 2. This case corresponds to a chronic HIV mono-infection but with unstimulated CTL-mediated immune response.

Scenario 3 (Stability of $\boldsymbol{\nabla}_{2}$ ): $\eta_{1}=0.0001, \quad \eta_{2}=0.01, \quad \sigma_{1}=0.001 \quad$ and $\sigma_{2}=0.05$. Then, we calculate $\mathfrak{R}_{1}=0.49<1<2.33=\mathfrak{R}_{2}, \mathfrak{R}_{4}=0.78<1$ and then $\mathfrak{R}_{1} / \mathfrak{R}_{2}=0.21<1$. Hence, the conditions in Table 2 are satisfied. The numerical results show that $\mathrm{Ð}_{2}=(421.67,0,0,89.2,1.37,0,0,0)$ exists. Figure 4 illustrates that the trajectories initiating with Initial-1, Initial-2 and Initial-3 tend to $Ð_{2}$. Thus, the numerical results consistent with Theorem 3. This situation leads to a persistent HTLV-I mono-infection with unstimulated CTL-mediated immune response.

Scenario 4 (Stability of $\boldsymbol{\boxplus}_{3}$ ): $\eta_{1}=0.001, \eta_{2}=0.005$ and $\sigma_{1}=\sigma_{2}=0.01$. Then, we calculate $\mathfrak{R}_{3}=1.39>1$ and $\mathfrak{R}_{5}=0.33<1$. Table 2 and Fig. 5 show that the trajectories initiating with Initial-1, Initial-2 and Initial-3 tend to $\mathrm{Ð}_{3}=(285.71,9.74,10,0,0,25,0.97,0)$. Therefore, $\mathrm{Ð}_{3}$ is G.A.S and this agrees with Theorem 4. Hence, a chronic HIV mono-infection with HIV-specific CTLmediated immune response is attained.

Scenario 5 (Stability of $\boldsymbol{\boxplus}_{4}$ ): $\eta_{1}=0.0007, \eta_{2}=0.1, \sigma_{1}=0.05$ and $\sigma_{2}=0.3$. Then, we calculate $\mathfrak{R}_{4}=5.37>1$ and $\mathfrak{R}_{6}=0.79<1$. According to Table $2, \mathrm{Ð}_{4}$ exists with $\bigoplus_{4}=(230.77,0,0,118.4,0.33,0,0,4.35)$. In Fig. 6, we show that the trajectories initiating with Initial-1, Initial-2 and Initial-3 tend to $\mathrm{Ð}_{4}$ and then it is G.A.S which agrees with Theorem 5. Hence, a chronic HTLV-I mono-infection with HTLV-specific CTL-mediated immune response is attained. 


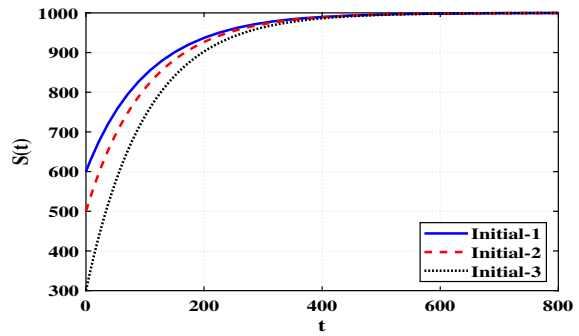

(a) Susceptible $\mathrm{CD} 4^{+} \mathrm{T}$ cells

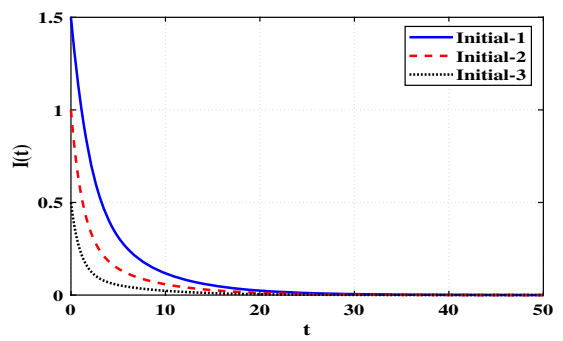

(c) Active HIV-infected cells

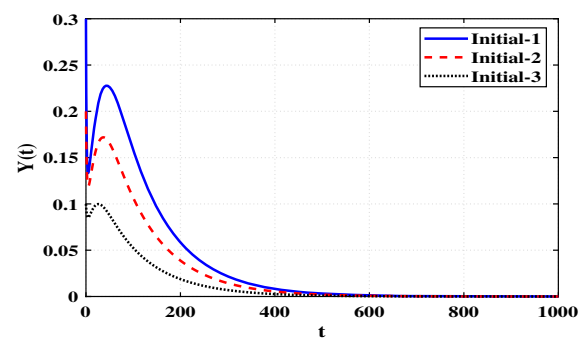

(e) Tax-expressing HTLV-infected cells

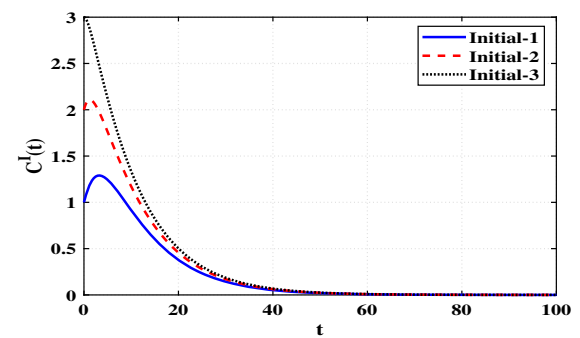

(g) HIV-specific CTLs

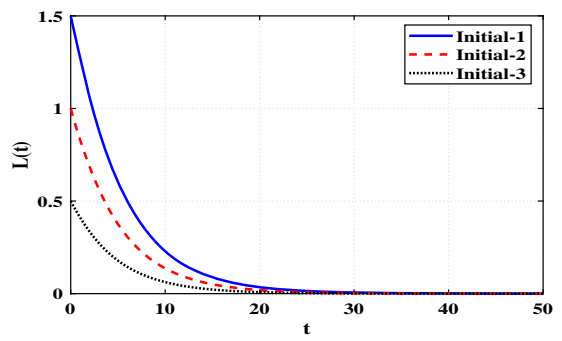

(b) Silent HIV-infected cells

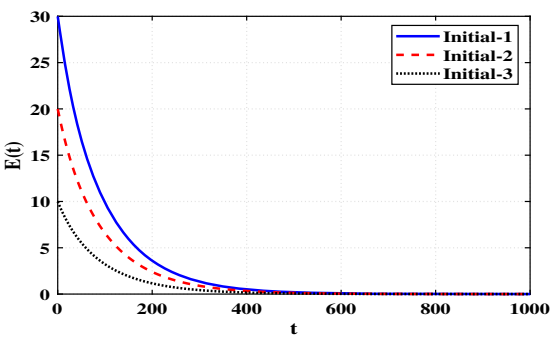

(d) Silent HTLV-infected cells

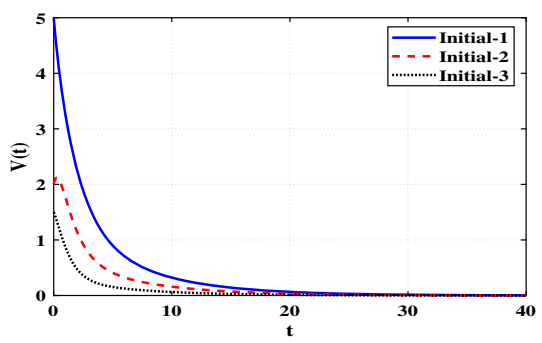

(f) Free HIV particles

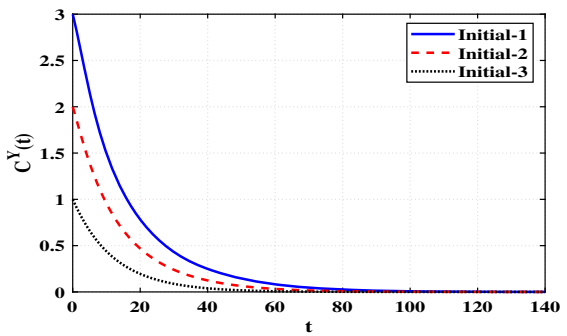

(h) HTLV-specific CTLs

Fig. 2 The behavior of solution trajectories of system (3) when $\mathfrak{R}_{1} \leq 1$ and $\mathfrak{R}_{2} \leq 1$

Scenario 6 (Stability of $\boldsymbol{D}_{5}$ ): $\eta_{1}=0.001, \quad \eta_{2}=0.01, \quad \sigma_{1}=0.05$ and $\sigma_{2}=0.08$. Then, we calculate $\mathfrak{R}_{5}=1.55>1, \quad \mathfrak{R}_{8}=0.85<1 \quad$ and $\mathfrak{R}_{1} / \mathfrak{R}_{2}=2.09>1$. Table 2 and the numerical results demonstrated in Fig. 7 


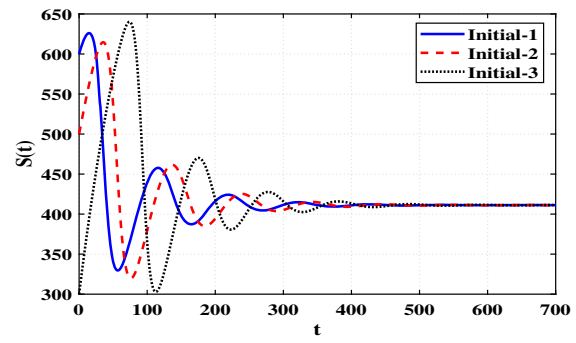

(a) Susceptible $\mathrm{CD} 4^{+} \mathrm{T}$ cells

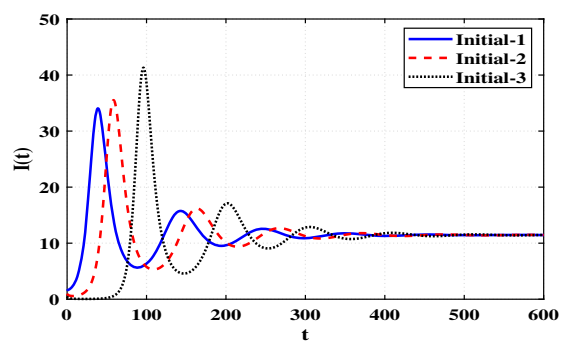

(c) Active HIV-infected cells

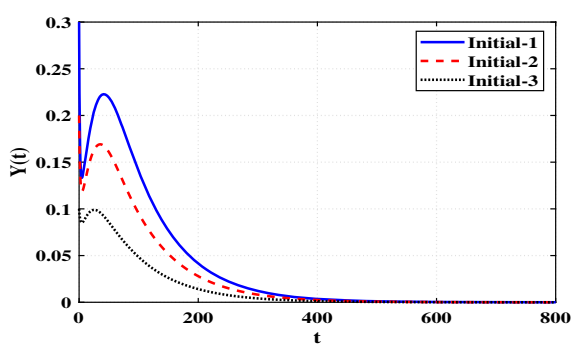

(e) Tax-expressing HTLV-infected cells

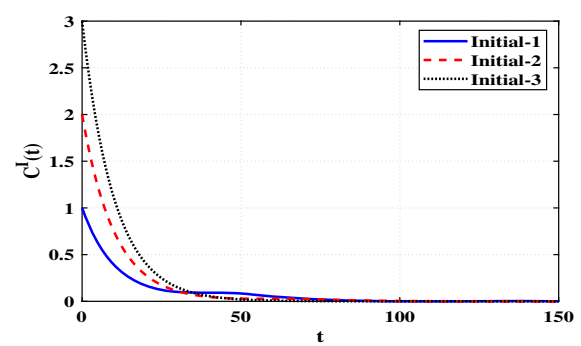

(g) HIV-specific CTLs

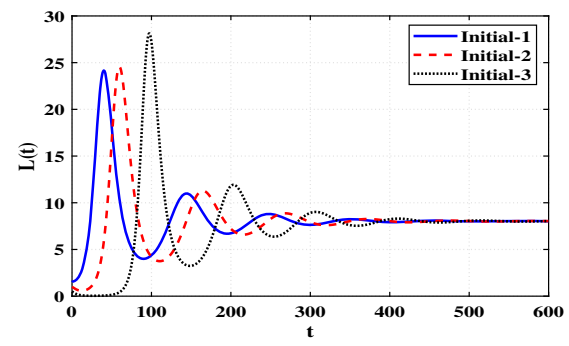

(b) Silent HIV-infected cells

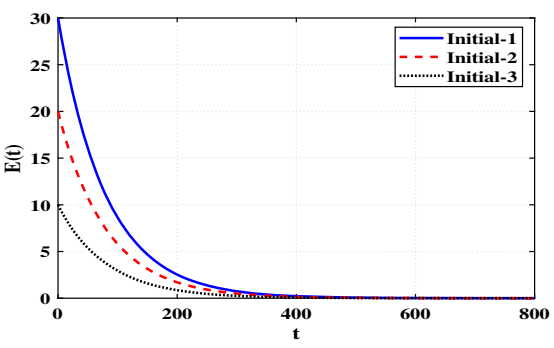

(d) Silent HTLV-infected cells

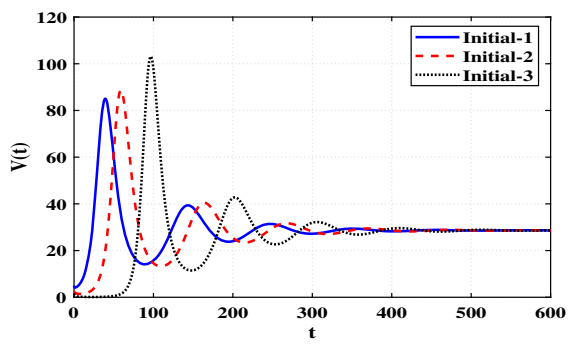

(f) Free HIV particles

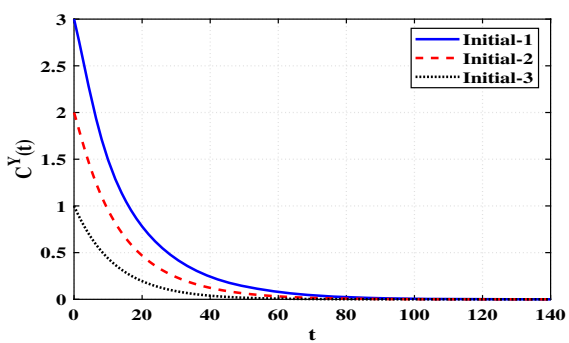

(h) HTLV-specific CTLs

Fig. 3 The behavior of solution trajectories of system (3) when $\mathfrak{R}_{1}>1, \mathfrak{R}_{2} / \mathfrak{R}_{1} \leq 1$ and $\mathfrak{R}_{3} \leq 1$

show that $\bigoplus_{5}=(421.67,2.88,2,56.68,0.87,5,2.63,0)$ exists and it is G.A.S and this agrees with Theorem 6. As a result, a chronic co-infection with HIV and HTLV-I is attained where the HIV-specific CTL-mediated immune 


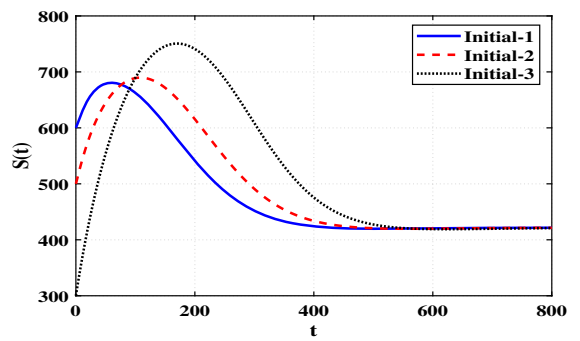

(a) Susceptible $\mathrm{CD} 4^{+} \mathrm{T}$ cells

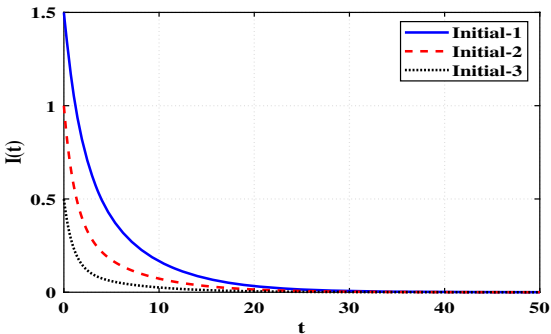

(c) Active HIV-infected cells

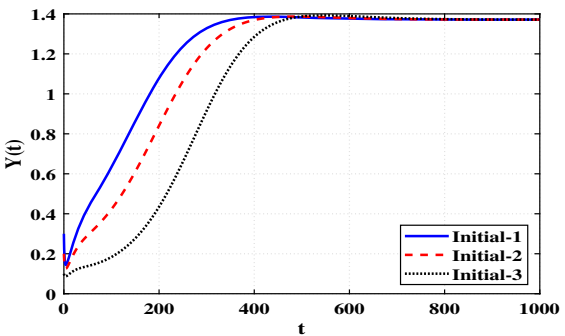

(e) Tax-expressing HTLV-infected cells

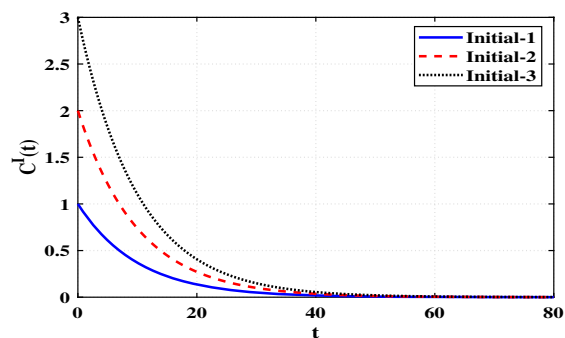

(g) HIV-specific CTLs

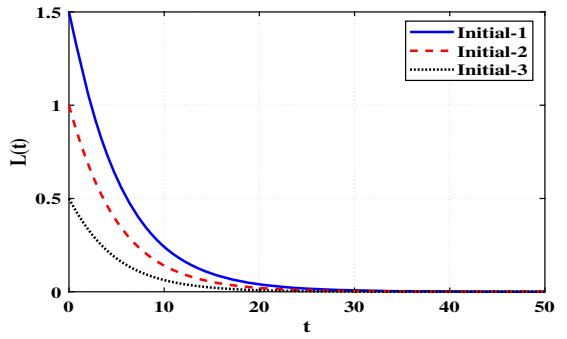

(b) Silent HIV-infected cells

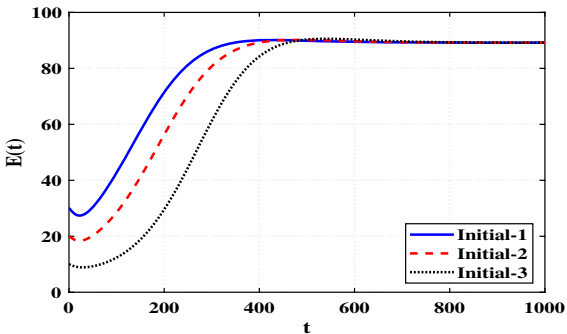

(d) Silent HTLV-infected cells

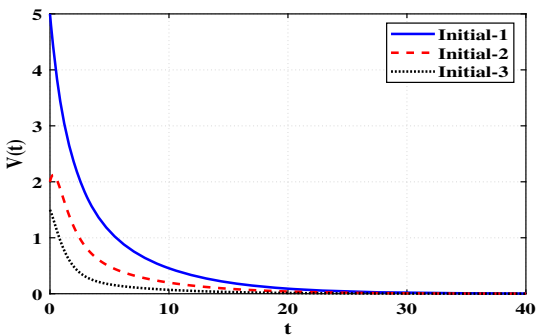

(f) Free HIV particles

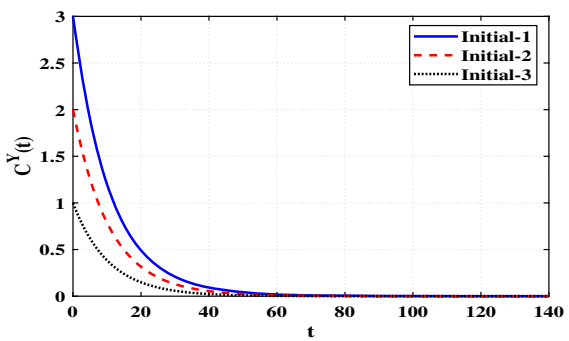

(h) HTLV-specific CTLs

Fig. 4 The behavior of solution trajectories of system (3) when $\mathfrak{R}_{2}>1, \mathfrak{R}_{1} / \mathfrak{R}_{2} \leq 1$ and $\mathfrak{R}_{4} \leq 1$

response is active and the HTLV-specific CTL-mediated immune response is unstimulated.

Scenario 7 (Stability of $\boldsymbol{\boxplus}_{6}$ ): $\eta_{1}=0.0006, \eta_{2}=0.04, \sigma_{1}=0.01$ and $\sigma_{2}=0.5$. We compute $\mathfrak{R}_{6}=1.62>1, \mathfrak{R}_{7}=0.88<1$ and $\mathfrak{R}_{2} / \mathfrak{R}_{1}=3.19>1$. Based on the 


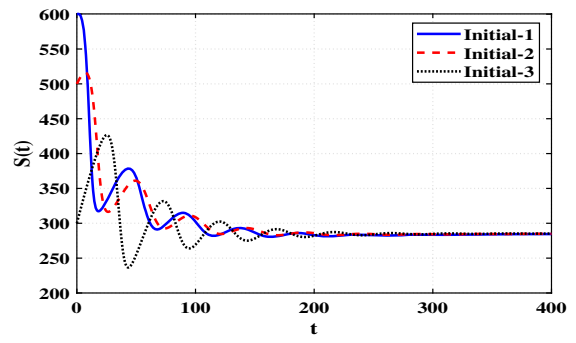

(a) Susceptible $\mathrm{CD} 4^{+} \mathrm{T}$ cells

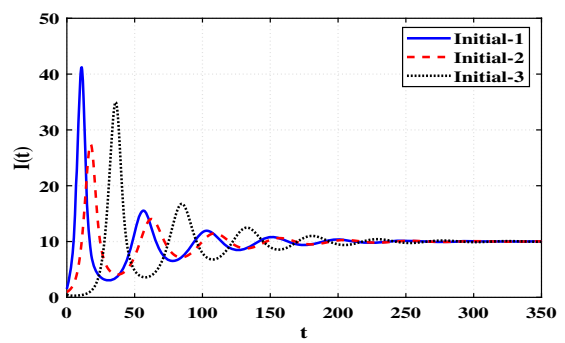

(c) Active HIV-infected cells

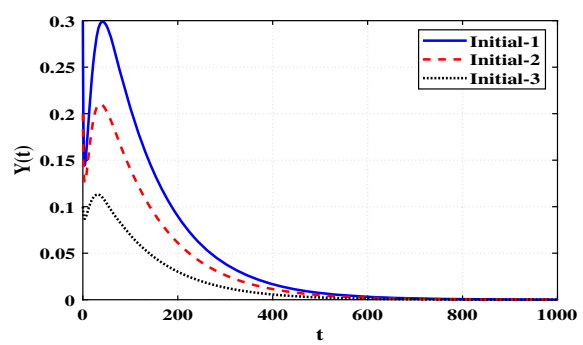

(e) Tax-expressing HTLV-infected cells

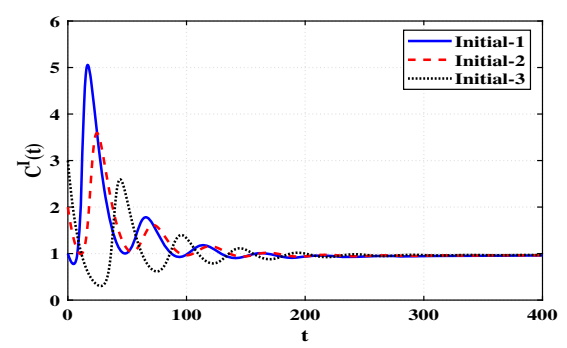

(g) HIV-specific CTLs

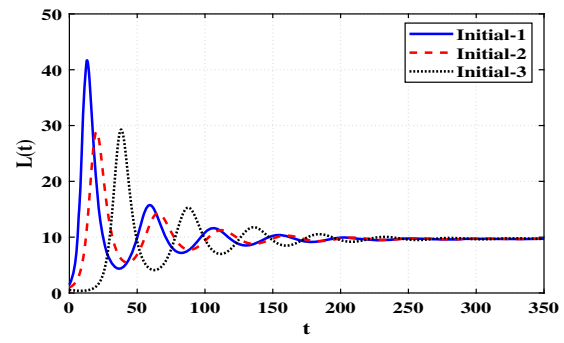

(b) Silent HIV-infected cells

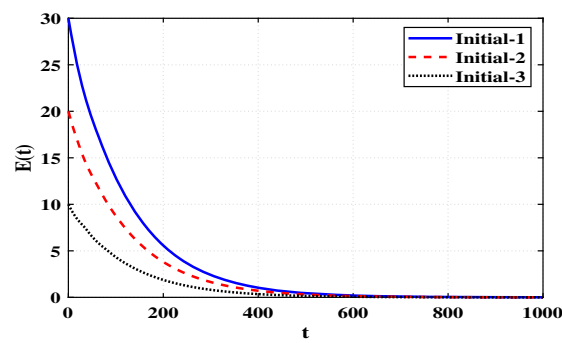

(d) Silent HTLV-infected cells

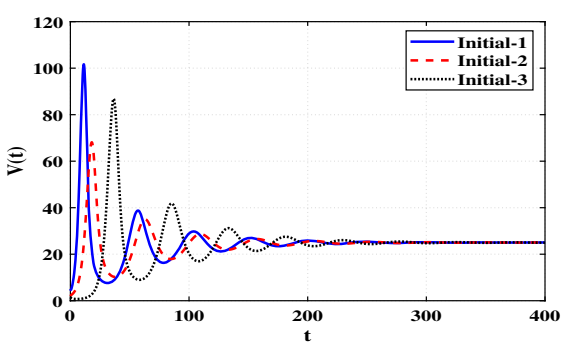

(f) Free HIV particles

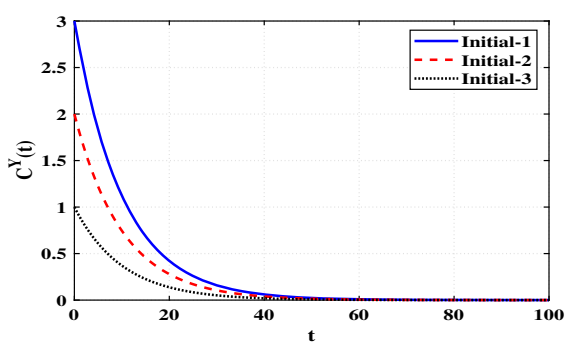

(h) HTLV-specific CTLs

Fig. 5 The behavior of solution trajectories of system (3) when $\mathfrak{R}_{3}>1$ and $\mathfrak{R}_{5} \leq 1$

conditions in Table 2, the equilibrium $\bigoplus_{6}=(342.68,5.23,7.45,42.21$, $0.2,18.64,0,2.19)$ exists. Moreover, the numerical results plotted in Fig. 8 show that $\mathrm{D}_{6}$ is G.A.S and this illustrates Theorem 7. As a result, a chronic co-infection with HIV and HTLV-I is attained where the HTLV-specific CTL-mediated immune 


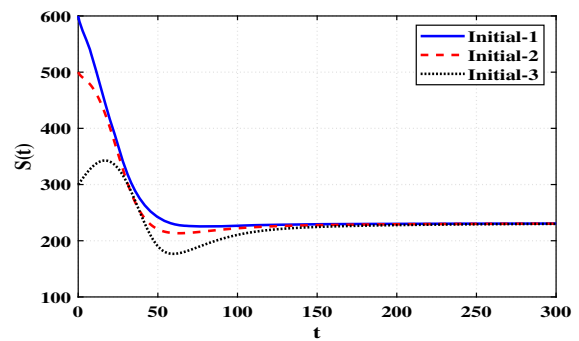

(a) Susceptible $\mathrm{CD} 4^{+} \mathrm{T}$ cells

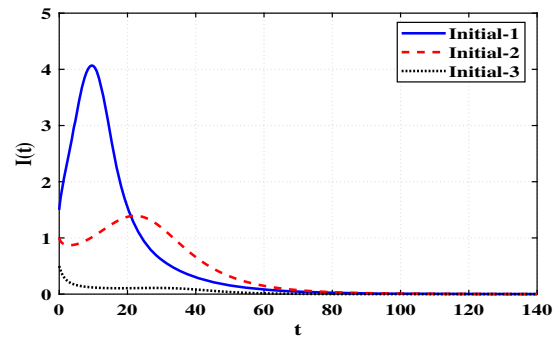

(c) Active HIV-infected cells

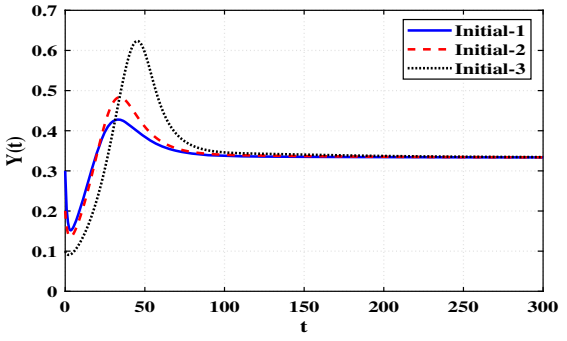

(e) Tax-expressing HTLV-infected cells

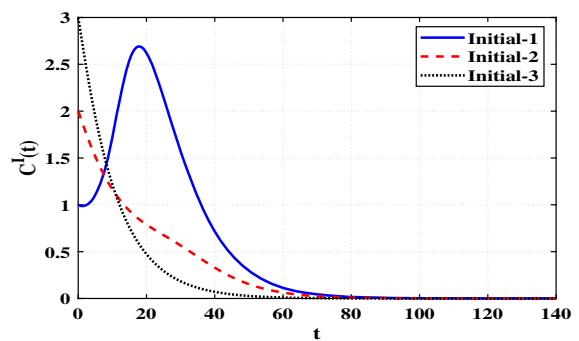

(g) HIV-specific CTLs

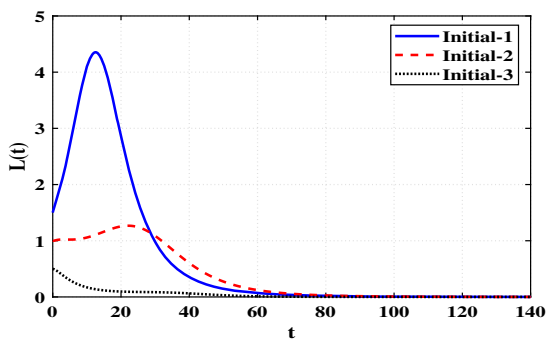

(b) Silent HIV-infected cells

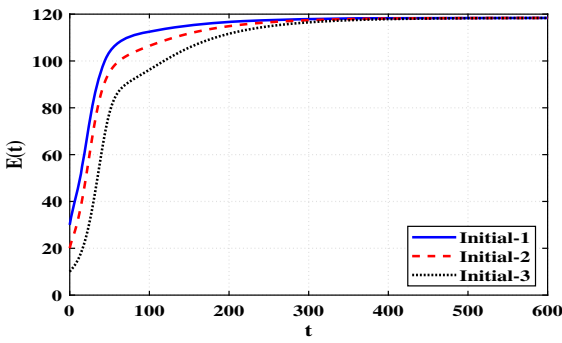

(d) Silent HTLV-infected cells

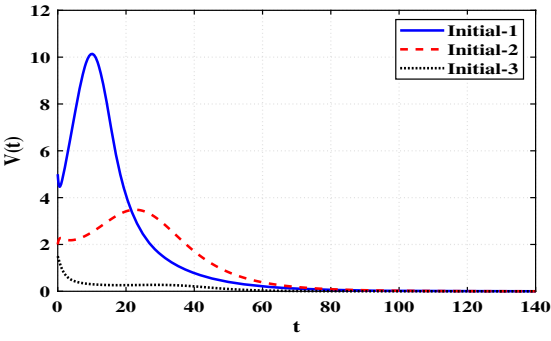

(f) Free HIV particles

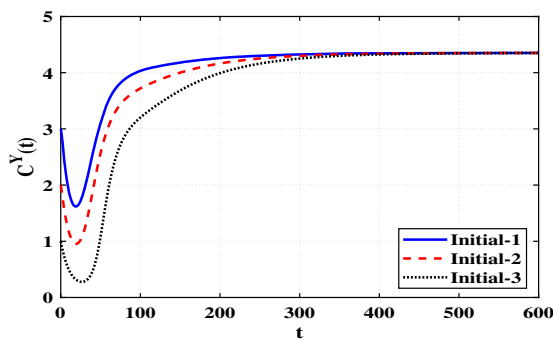

(h) HTLV-specific CTLs

Fig. 6 The behavior of solution trajectories of system (3) when $\mathfrak{R}_{4}>1$ and $\mathfrak{R}_{6} \leq 1$

response is active and the $\mathrm{HIV}$-specific CTL-mediated immune response is unstimulated.

Scenario 8 (Stability of $\boldsymbol{D}_{7}$ ): $\eta_{1}=0.0006, \eta_{2}=0.04, \sigma_{1}=0.05$ and $\sigma_{2}=0.5$. These data give $\mathfrak{R}_{7}=1.39>1$ and $\mathfrak{R}_{8}=4.43>1$. According to Table 2 , the 


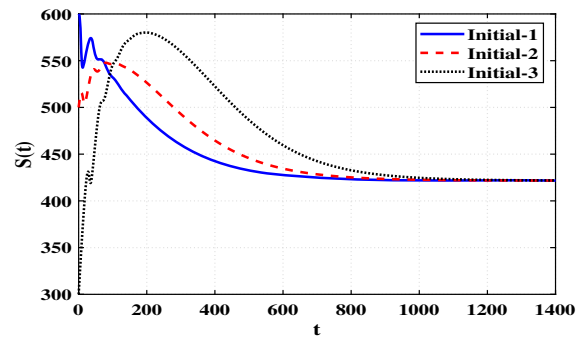

(a) Susceptible $\mathrm{CD} 4^{+} \mathrm{T}$ cells

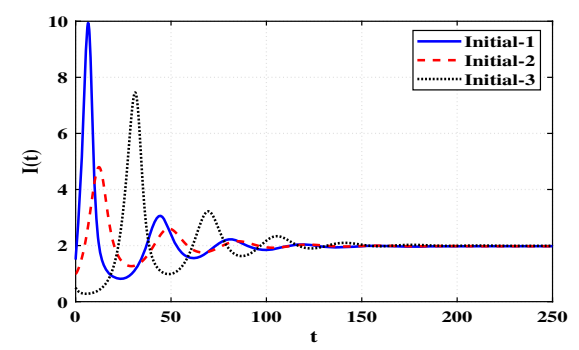

(c) Active HIV-infected cells

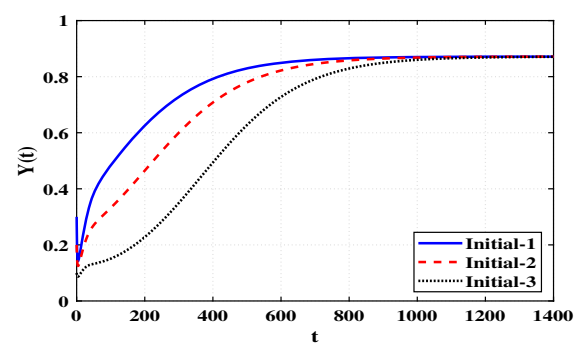

(e) Tax-expressing HTLV-infected cells

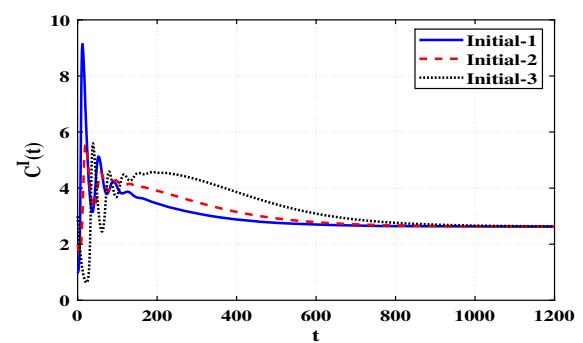

(g) HIV-specific CTLs

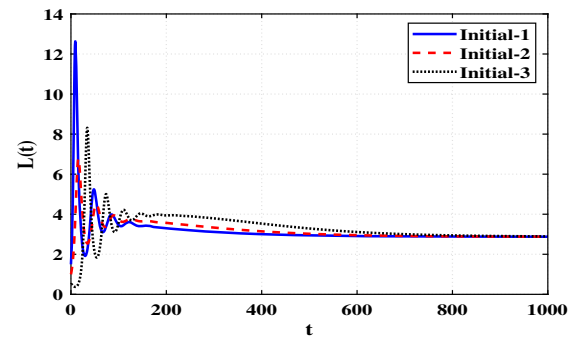

(b) Silent HIV-infected cells

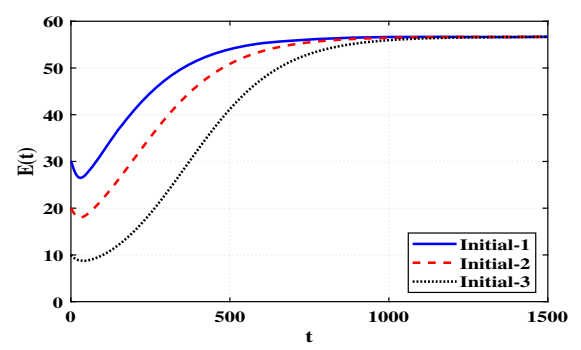

(d) Silent HTLV-infected cells

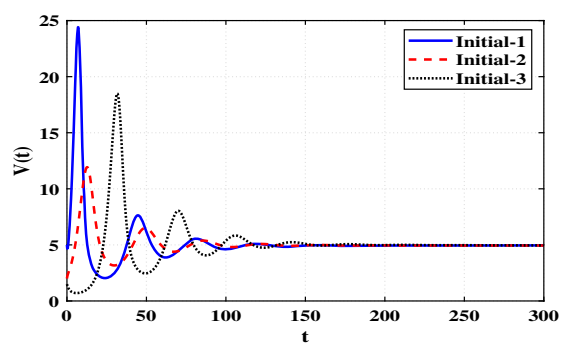

(f) Free HIV particles

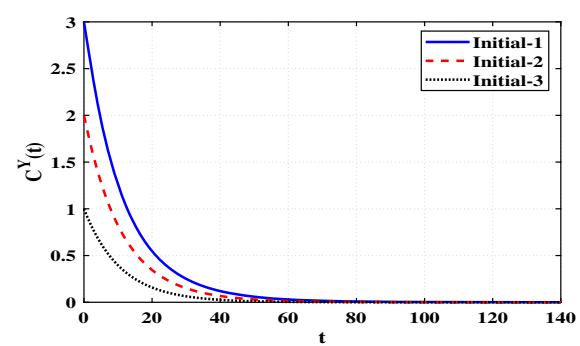

(h) HTLV-specific CTLs

Fig. 7 The behavior of solution trajectories of system (3) when $\mathfrak{R}_{5}>1, \mathfrak{R}_{8} \leq 1$ and $\mathfrak{R}_{1} / \mathfrak{R}_{2}>1$

equilibrium $\mathrm{Ð}_{7}$ exists. Figure 9 illustrates that the trajectories initiating with Initial1 , Initial-2 and Initial-3 tend to $\bigoplus_{7}=(476.19,1.95,2,58.64,0.2,5,0.97,3.42)$. The numerical results displayed in Fig. 9 show that $\mathrm{D}_{7}$ is G.A.S based on Theorem 8. In this case, a chronic co-infection with HIV and HTLV-I is attained where both HIV- 
specific CTL-mediated and HTLV-specific CTL-mediated immune responses are working.

To further confirmation, we calculate the Jacobian matrix $J=$ $J\left(S, L, I, E, Y, V, C^{I}, C^{Y}\right)$ of system (3) as in the following form:

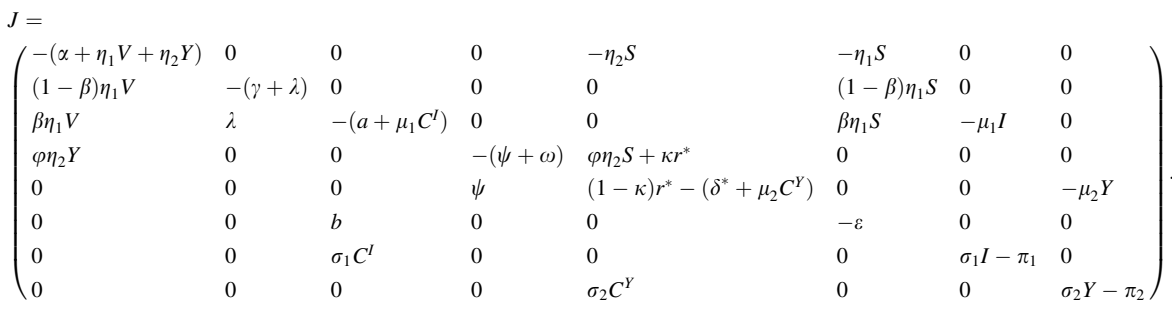

Then, we calculate the eigenvalues $\lambda_{i}, i=1,2, \ldots, 8$ of the matrix $J$ at each equilibrium. The examined steady will be locally stable if all its eigenvalues satisfy the following condition:

$$
\operatorname{Re}\left(\lambda_{i}\right)<0, i=1,2, \ldots, 8 .
$$

We use the parameters $\eta_{1}, \eta_{2}, \sigma_{1}$ and $\sigma_{2}$ the same as given above to compute all positive equilibria and the corresponding eigenvalues. From the scenarios 1-8, we present in Table 5 the positive equilibria, the real parts of the eigenvalues and whether the equilibrium is locally stable or unstable. The numerical results are consistent with the global stability results. For each of the above mentioned scenarios, only one equilibrium is asymptotically stable, while the others are unstable.

\subsection{Comparison results}

In this subsection, we study the influence of HTLV-I infection on HIV monoinfection dynamics, and how affect the HIV infection on the dynamics of HTLV-I mono-infection as well.

\section{Impact of HTLV-I infection on HIV mono-infection dynamics}

To investigate the effect of HTLV-I infection on HIV mono-infection dynamics, we make a comparison between model (3) and the following HIV mono-infection model:

$$
\left\{\begin{array}{l}
\dot{S}=\rho-\alpha S-\eta_{1} S V, \\
\dot{L}=(1-\beta) \eta_{1} S V-(\lambda+\gamma) L, \\
\dot{I}=\beta \eta_{1} S V+\lambda L-a I-\mu_{1} C^{I} I, \\
\dot{V}=b I-\varepsilon V, \\
\dot{C}^{I}=\sigma_{1} C^{I} I-\pi_{1} C^{I} .
\end{array}\right.
$$

We fix parameters $\eta_{1}=0.0006, \sigma_{1}=0.05$, and $\sigma_{2}=0.5$ and consider the following initial condition:

Initial-4: $\quad\left(S(0), L(0), I(0), E(0), Y(0), V(0), C^{I}(0), C^{Y}(0)\right)=(600,2.4,1.8,60$, $0.2,4.5,1.8,3.5)$. 
We choose two values of the parameter $\eta_{2}$ as $\eta_{2}=0.04$ (HIV/HTLV-I coinfection), and $\eta_{2}=0.0$ (HIV mono-infection). It can be seen from Fig. 10 that when the HIV mono-infected individual is co-infected with HTLV-I then the concentrations of susceptible $\mathrm{CD}^{+}{ }^{+} \mathrm{T}$ cells, silent HIV-infected cells and HIV-

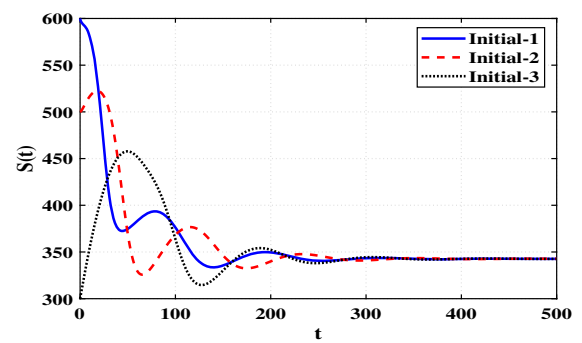

(a) Susceptible $\mathrm{CD} 4^{+} \mathrm{T}$ cells

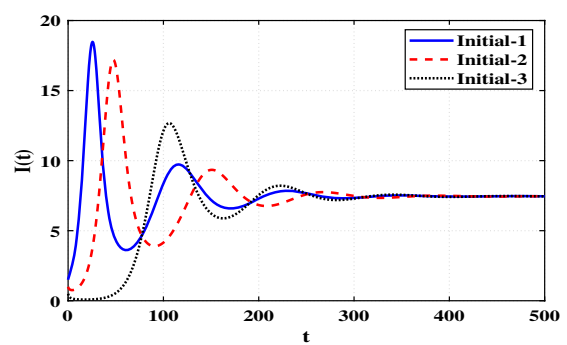

(c) Active HIV-infected cells

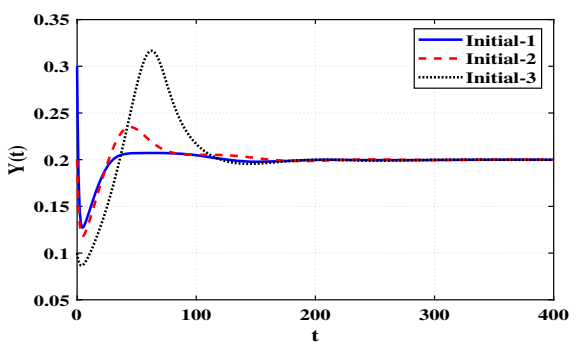

(e) Tax-expressing HTLV-infected cells

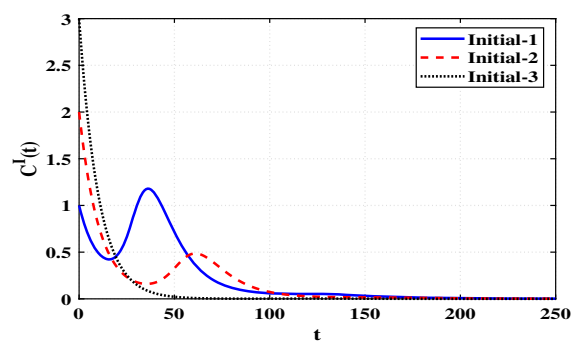

(g) HIV-specific CTLs

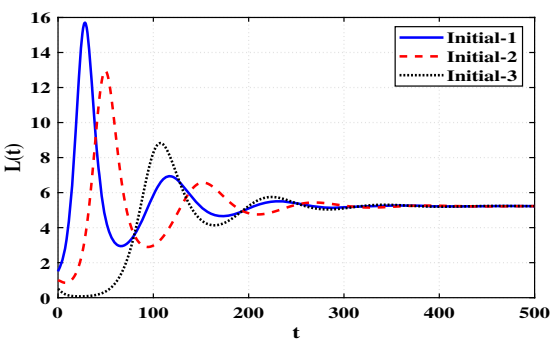

(b) Silent HIV-infected cells

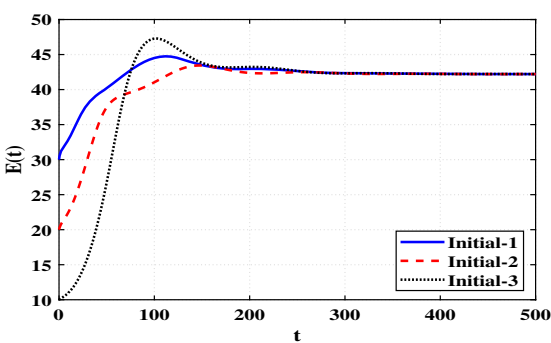

(d) Silent HTLV-infected cells

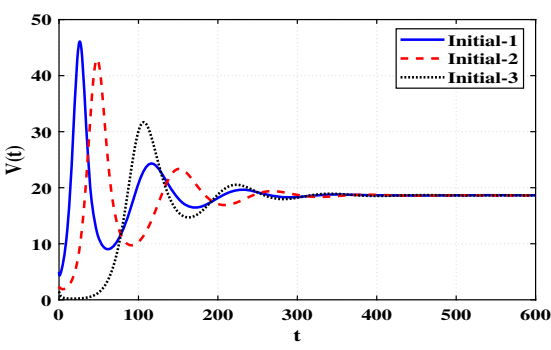

(f) Free HIV particles

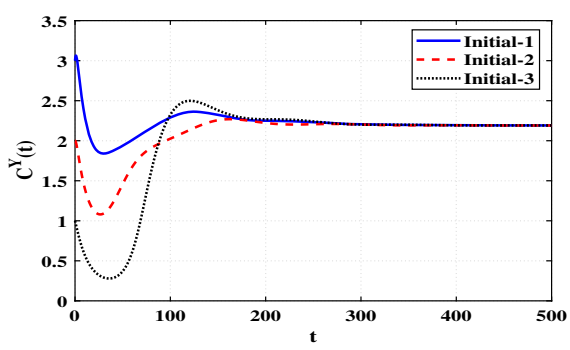

(h) HTLV-specific CTLs

Fig. 8 The behavior of solution trajectories of system (3) when $\mathfrak{R}_{6}>1, \mathfrak{R}_{7} \leq 1$ and $\mathfrak{R}_{2} / \mathfrak{R}_{1}>1$ 


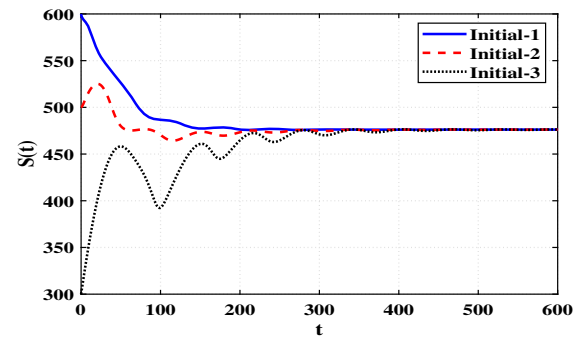

(a) Susceptible $\mathrm{CD} 4^{+} \mathrm{T}$ cells

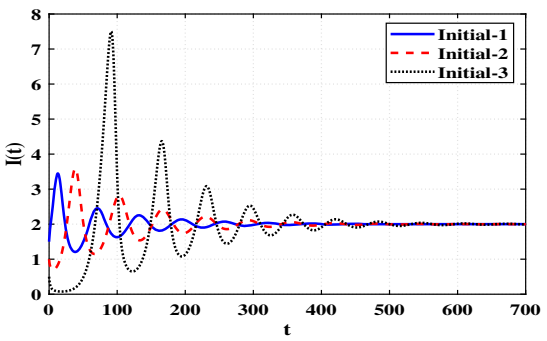

(c) Active HIV-infected cells

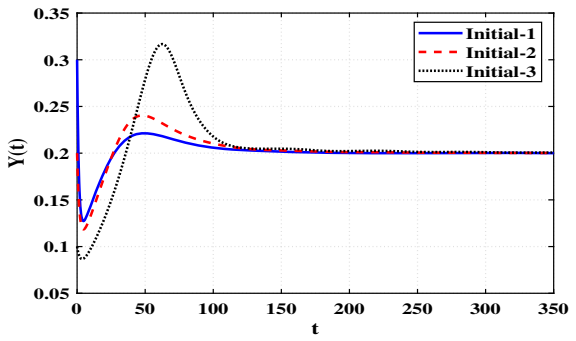

(e) Tax-expressing HTLV-infected cells

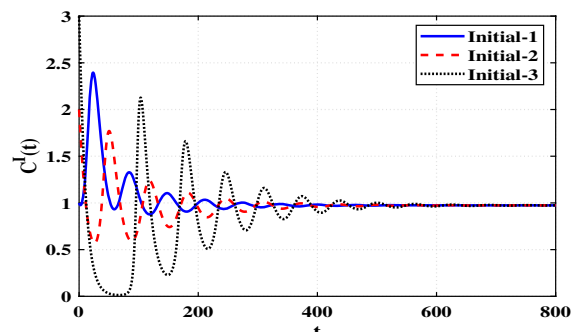

(g) HIV-specific CTLs

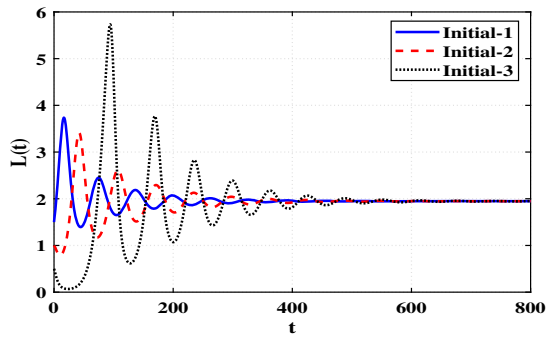

(b) Silent HIV-infected cells

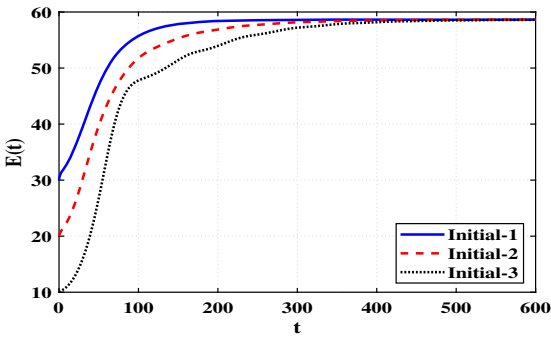

(d) Silent HTLV-infected cells

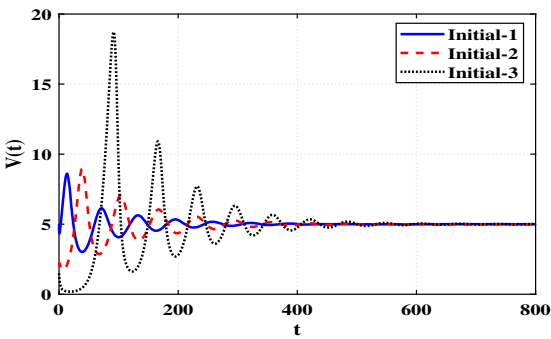

(f) Free HIV particles

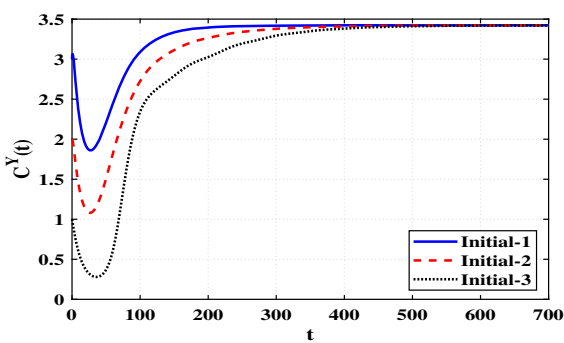

(h) HTLV-specific CTLs

Fig. 9 The behavior of solution trajectories of system (3) when $\mathfrak{R}_{7}>1$ and $\mathfrak{R}_{8}>1$

specific CTLs are decreased. Although, the concentration of free HIV particles tend to the same value in both HIV mono-infection and HIV/HTLV-I co-infection. Indeed, such observation is compatible with the study that has been performed by Vandormael et al. in 2017 [61]. The researchers have not found any worthy 
differences in the concentration of HIV virus particles in comparison between HIV mono-infected and HIV/HTLV-I co-infected patients.

\section{Impact of HIV infection on HTLV-I mono-infection dynamics}

To investigate the effect of HIV infection on HTLV-I mono-infection dynamics, we make a comparison between model (3) and the following HTLV-I monoinfection model:

$$
\left\{\begin{array}{l}
\dot{S}=\rho-\alpha S-\eta_{2} S Y \\
\dot{E}=\varphi \eta_{2} S Y+\kappa r^{*} Y-(\psi+\omega) E \\
\dot{Y}=\psi E+(1-\kappa) r^{*} Y-\delta^{*} Y-\mu_{2} C^{Y} Y \\
\dot{C}^{Y}=\sigma_{2} C^{Y} Y-\pi_{2} C^{Y}
\end{array}\right.
$$

We fix parameters $\eta_{2}=0.01 ; \sigma_{1}=0.05$, and $\sigma_{2}=0.5$ and consider the following initial condition:

Initial-5: $\quad\left(S(0), L(0), I(0), E(0), Y(0), V(0), C^{I}(0), C^{Y}(0)\right)=(700,4,2,21$, $0.198,5,4.5,0.6)$.

We choose two values of the parameter $\eta_{1}$ as $\eta_{1}=0.001$ (HIV/HTLV-I coinfection), and $\eta_{1}=0.0$ (HTLV-I mono-infection). It can be seen from Fig. 11 that when the HTLV-I mono-infected individual is co-infected with HIV then the concentrations of susceptible $\mathrm{CD}^{+}{ }^{+} \mathrm{T}$ cells, silent HTLV-infected cells and HTLVspecific CTLs are decreased. Although, the concentration of Tax-expressing HTLVinfected cells tend to the same value in both HTLV-I mono-infection and HIV/ HTLV-I co-infection.

\section{Conclusion and discussions}

This paper investigates the global behavior of solutions of system that was used to study HIV/HTLV-I co-infection dynamics. We incorporated the effect of HIVspecific CTLs and HTLV-specific CTLs into the model. The HIV can be transmitted to the susceptible $\mathrm{CD} 4^{+} \mathrm{T}$ cells by virus-to-cell transmission, while HTLV-I has two modes of transmission, (i) horizontal transmission via direct cell-to-cell contact, and (ii) vertical transmission through mitotic division of Tax-expressing HTLV-infected cells. We studied the basic properties of the model by showing that the solutions are nonnegative and bounded. We derived eight threshold parameters that governed the existence and stability of the eight equilibria of the model. We constructed suitable Lyapunov functions and utilized Lyapunov-LaSalle asymptotic stability theorem to establish the global asymptotic stability of all equilibria. We conducted numerical simulations to support and clarify our theoretical results. We studied the effect of HIV infection on HTLV-I mono-infection dynamics and vice versa. The model analysis suggested that co-infected individuals with both viruses will have smaller number of healthy $\mathrm{CD}^{+}{ }^{+} \mathrm{T}$ cells in comparison with HIV or HTLV-I monoinfected individuals. It was reported in [62] that no treatments exist for acute or chronic HTLV-I infection. However, antiviral treatments of HIV infection is currently used to suppress viral replication. For example, reverse transcriptase inhibitors (RTIs) can prevent the establishment of productive infection of a cell. Model (4) under the effect of RTIs can be written as: 


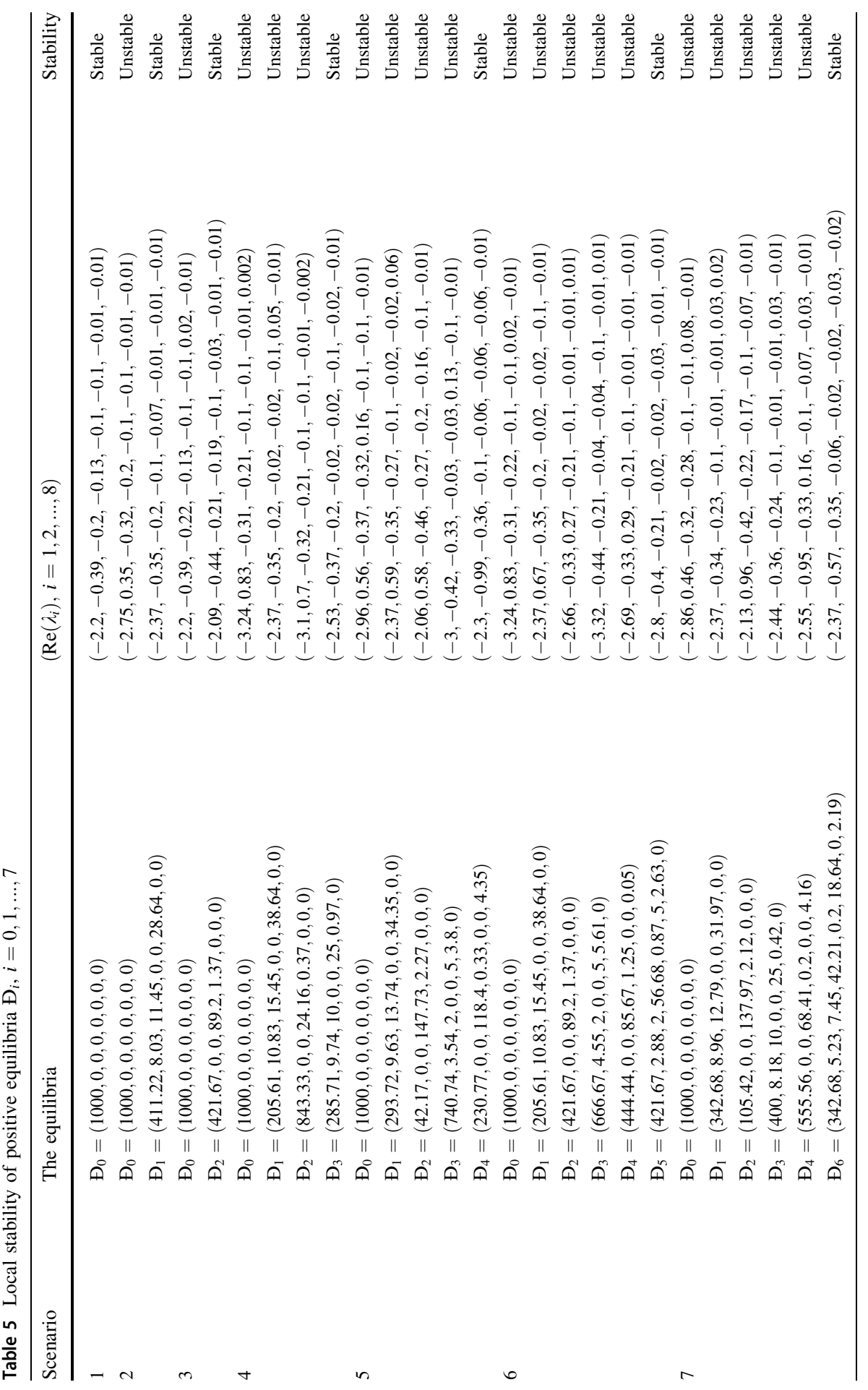




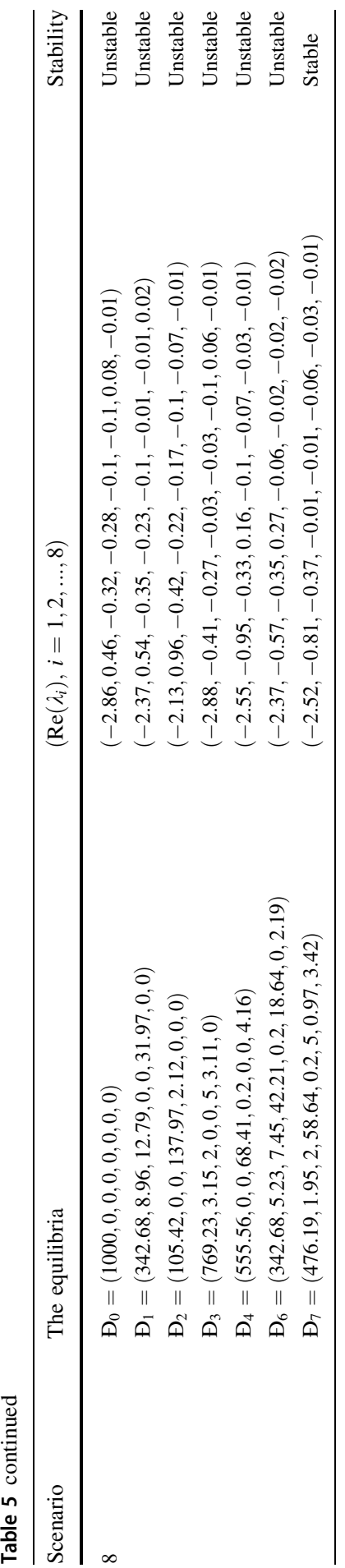




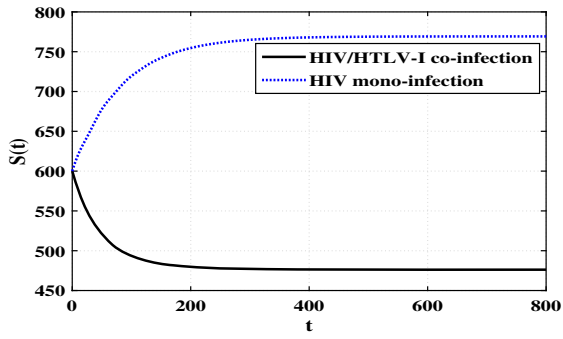

(a) Susceptible $\mathrm{CD} 4^{+} \mathrm{T}$ cells

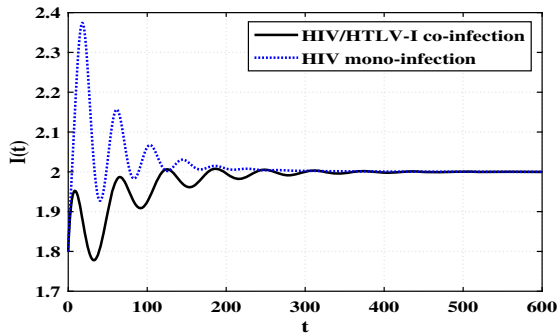

(c) Active HIV-infected cells

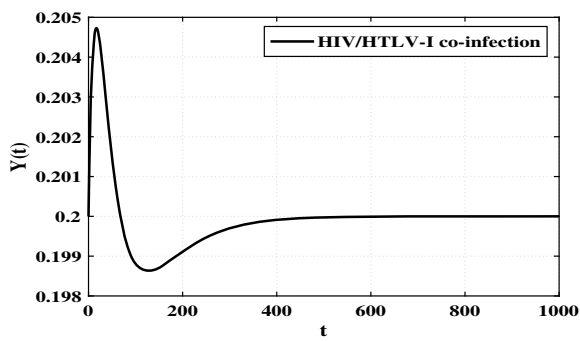

(e) Tax-expressing HTLV-infected cells

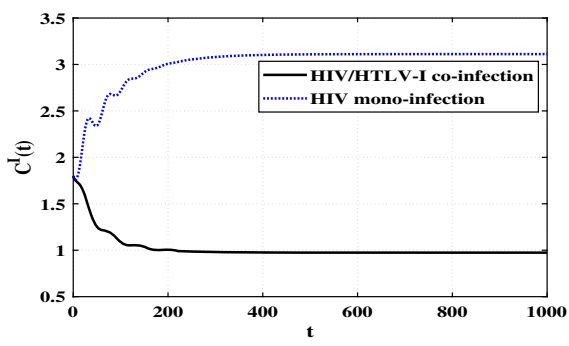

(g) HIV-specific CTLs

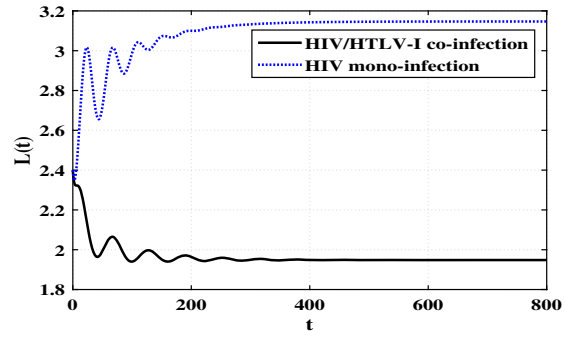

(b) Silent HIV-infected cells

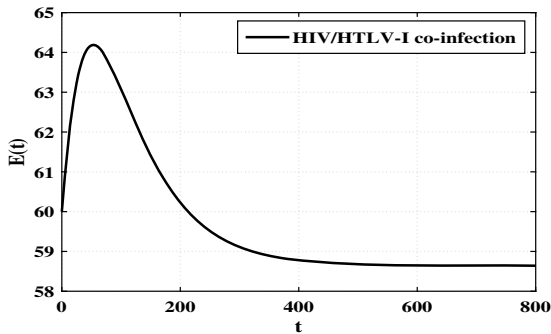

(d) Silent HTLV-infected cells

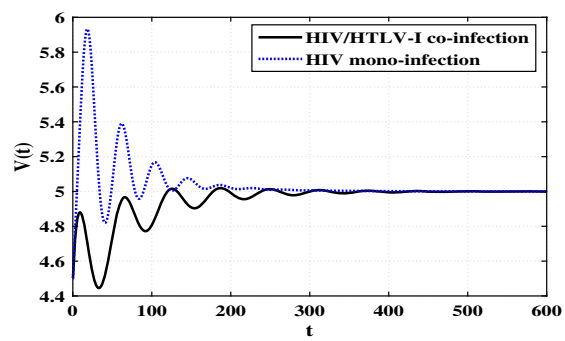

(f) Free HIV particles

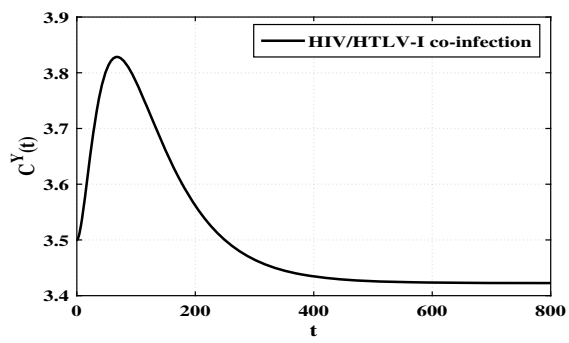

(h) HTLV-specific CTLs

Fig. 10 The influence of HTLV-I infection rate $\left(\eta_{2} \neq 0\right)$ on HIV mono-infection dynamics (22) will cause a chronic HIV/HTLV-I co-infection 


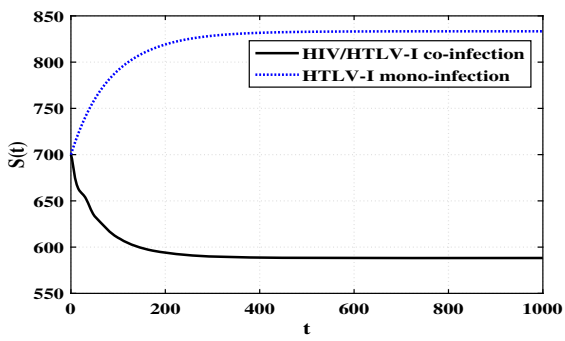

(a) Susceptible $\mathrm{CD} 4^{+} \mathrm{T}$ cells

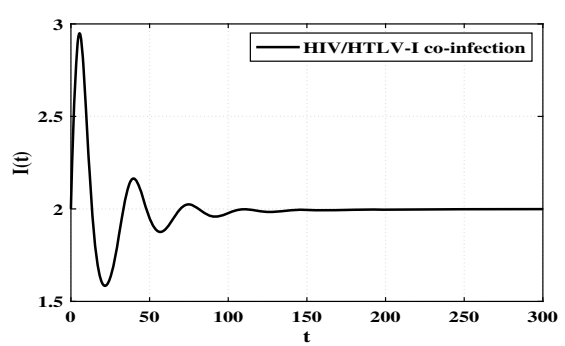

(c) Active HIV-infected cells

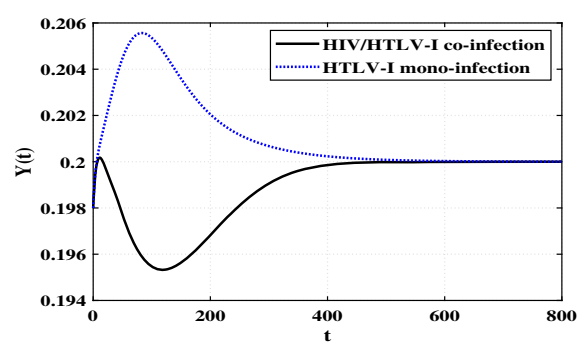

(e) Tax-expressing HTLV-infected cells

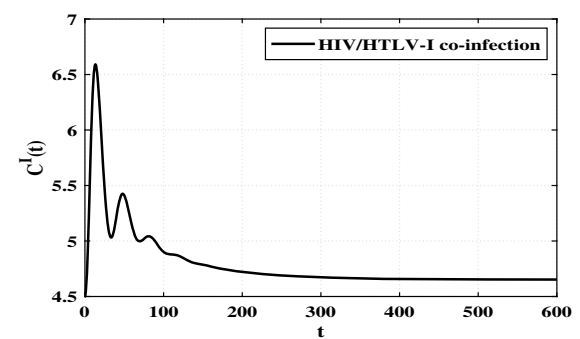

(g) HIV-specific CTLs

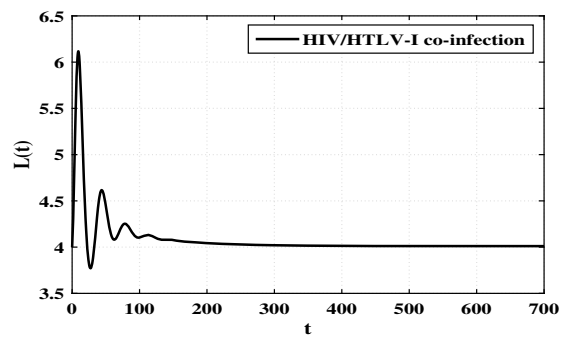

(b) Silent HIV-infected cells

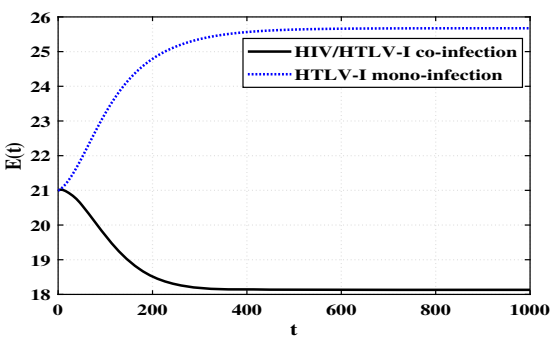

(d) Silent HTLV-infected cells

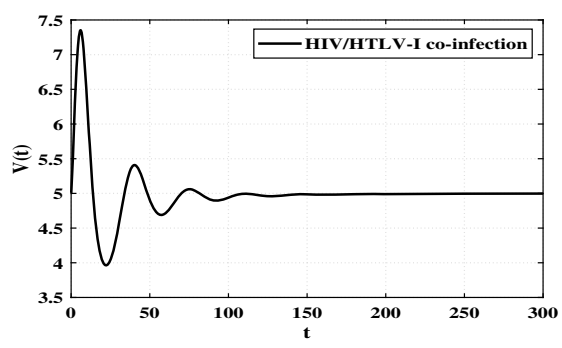

(f) Free HIV particles

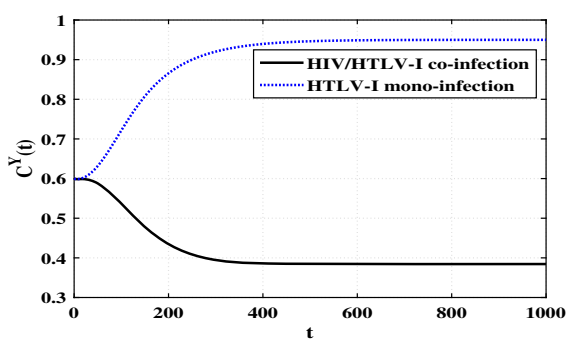

(h) HTLV-specific CTLs

Fig. 11 The influence of HIV infection rate $\left(\eta_{1} \neq 0\right)$ on HTLV mono-infection dynamics (23) will cause a chronic HIV/HTLV-I co-infection 


$$
\left\{\begin{array}{l}
\dot{S}=\rho-\alpha S-(1-\ell) \eta_{1} S V-\eta_{2} S Y \\
\dot{L}=(1-\ell)(1-\beta) \eta_{1} S V-(\lambda+\gamma) L, \\
\dot{I}=(1-\ell) \beta \eta_{1} S V+\lambda L-a I-\mu_{1} C^{I} I, \\
\dot{E}=\varphi \eta_{2} S Y+r Y-(\psi+\omega) E \\
\dot{Y}=\psi E-\delta Y-\mu_{2} C^{Y} Y, \\
\dot{V}=b I-\varepsilon V \\
\dot{C}^{I}=\sigma_{1} C^{I} I-\pi_{1} C^{I} \\
\dot{C}^{Y}=\sigma_{2} C^{Y} Y-\pi_{2} C^{Y}
\end{array}\right.
$$

where $\ell \in[0,1]$ is the effectiveness of RTIs. We noted that the threshold parameters $\mathfrak{R}_{2}$ and $\mathfrak{R}_{4}$ does not depend on the effectiveness of RTIs $\ell$, while the remaining threshold parameters depend on $\ell$ as follows:

$$
\begin{aligned}
& \mathfrak{R}_{1}=\frac{(1-\ell) \eta_{1} b S_{0}(\beta \gamma+\lambda)}{a \varepsilon(\gamma+\lambda)}, \quad \mathfrak{R}_{3}=\frac{(1-\ell) \sigma_{1} \rho \eta_{1} b(\beta \gamma+\lambda)}{a(\gamma+\lambda)\left((1-\ell) \pi_{1} \eta_{1} b+\alpha \varepsilon \sigma_{1}\right)}, \\
& \mathfrak{R}_{5}=\frac{\rho \varphi \varepsilon \eta_{2} \sigma_{1} \psi}{[(\delta-r) \psi+\delta \omega]\left((1-\ell) \pi_{1} \eta_{1} b+\alpha \varepsilon \sigma_{1}\right)}, \mathfrak{R}_{6}=\frac{(1-\ell) \rho b \eta_{1} \sigma_{2}(\beta \gamma+\lambda)}{a \varepsilon(\gamma+\lambda)\left(\pi_{2} \eta_{2}+\alpha \sigma_{2}\right)}, \\
& \mathfrak{R}_{7}=\frac{(1-\ell) \eta_{1} b \sigma_{1} \sigma_{2} \rho(\beta \gamma+\lambda)}{a(\gamma+\lambda)\left((1-\ell) \pi_{1} \eta_{1} b \sigma_{2}+\pi_{2} \eta_{2} \varepsilon \sigma_{1}+\alpha \varepsilon \sigma_{1} \sigma_{2}\right)}, \\
& \mathfrak{R}_{8}=\frac{\psi \varphi \eta_{2} \varepsilon \sigma_{1} \sigma_{2} \rho}{[(\delta-r) \psi+\delta \omega)]\left((1-\ell) \pi_{1} \eta_{1} b \sigma_{2}+\pi_{2} \eta_{2} \varepsilon \sigma_{1}+\alpha \varepsilon \sigma_{1} \sigma_{2}\right)} .
\end{aligned}
$$

Therefore to clear the HIV from the body by RTIs we have three cases:

(i) $\mathfrak{R}_{1} \leq 1$ and $\mathfrak{R}_{2} \leq 1$ : Let $\ell$ be chosen such that

$$
\begin{aligned}
& \mathfrak{R}_{1}=\frac{(1-\ell) \eta_{1} b S_{0}(\beta \gamma+\lambda)}{a \varepsilon(\gamma+\lambda)} \leq 1, \\
& \ell \geq \ell_{1}^{\min }=\max \left\{0,1-\frac{a \varepsilon(\gamma+\lambda)}{\eta_{1} b S_{0}(\beta \gamma+\lambda)}\right\} .
\end{aligned}
$$

It follows that when $\ell \geq \ell_{1}^{\min }$ and $\mathfrak{R}_{2} \leq 1$, the system will converge to $\bigoplus_{0}$ where both HIV and HTLV-I will be cleared from the body.

(ii) $\mathfrak{R}_{2}>1, \mathfrak{R}_{4} \leq 1$ and $\mathfrak{R}_{1} / \mathfrak{R}_{2} \leq 1$ : Let $\ell$ be chosen such that

$$
\begin{aligned}
\mathfrak{R}_{1} / \mathfrak{R}_{2} & =\frac{(1-\ell) \eta_{1} b(\beta \gamma+\lambda)[(\delta-r) \psi+\delta \omega]}{a \varepsilon \varphi \eta_{2} \psi(\gamma+\lambda)} \leq 1, \\
& \Longrightarrow \ell \geq \ell_{2}^{\min }=\max \left\{0,1-\frac{a \varepsilon \varphi \eta_{2} \psi(\gamma+\lambda)}{\eta_{1} b(\beta \gamma+\lambda)[(\delta-r) \psi+\delta \omega]}\right\} .
\end{aligned}
$$

Thus, if $\mathfrak{R}_{2}>1, \mathfrak{R}_{4} \leq 1$ and $\ell \geq \ell_{2}^{\text {min }}$, then the system will converge to $\mathrm{Ð}_{2}$ where the HIV is cleared while the HTLV-I will be chronic with inactive HTLV-specific CTL immune response.

(iii) $\mathfrak{R}_{4}>1$ and $\mathfrak{R}_{6} \leq 1$. Let $\ell$ is chosen such that $\mathfrak{R}_{6} \leq 1$ as: 


$$
\begin{aligned}
\mathfrak{R}_{6} & =\frac{(1-\ell) \rho b \eta_{1} \sigma_{2}(\beta \gamma+\lambda)}{a \varepsilon(\gamma+\lambda)\left(\pi_{2} \eta_{2}+\alpha \sigma_{2}\right)} \leq 1, \\
& \Longrightarrow \ell \geq \ell_{3}^{\min }=\max \left\{0,1-\frac{a \varepsilon(\gamma+\lambda)\left(\pi_{2} \eta_{2}+\alpha \sigma_{2}\right)}{\rho b \eta_{1} \sigma_{2}(\beta \gamma+\lambda)}\right\} .
\end{aligned}
$$

Therefore, if $\mathfrak{R}_{4}>1$ and $\ell \geq \ell_{3}^{\min }$, then the system will converge to $\mathrm{Ð}_{4}$ where the HIV is cleared while the HTLV-I will be chronic with active HTLV-specific CTL immune response.

\section{References}

1. WHO: Global Health Observatory (GHO) data. HIV/AIDS. http://www.who.int/gho/hiv/en/ (2018).

2. Nowak, M.A., Bangham, C.R.M.: Population dynamics of immune responses to persistent viruses. Science 272(5258), 74-79 (1996)

3. Hernandez-Vargas, E.A.: Modeling kick-kill strategies toward HIV cure. Front. Immunol. 8, Article ID: 995 (2017)

4. Pedro, K.D., Henderson, A.J., Agosto, L.M.: Mechanisms of HIV-1 cell-to-cell transmission and the establishment of the latent reservoir. Virus Res. 265, 115-121 (2019)

5. Rong, L., Perelson, A.S.: Modeling latently infected cell activation: viral and latent reservoir persistence, and viral blips in HIV-infected patients on potent therapy, Journal of Theoretical Biology. PLoS Comput. Biol. 5(10), e1000533 (2009)

6. Perelson, A.S., Essunger, P., Cao, Y., Vesanen, M., Hurley, A., Saksela, K., Markowitz, M., Ho, D.D.: Decay characteristics of HIV-1-infected compartments during combination therapy. Nature 387(6629), 188-191 (1997)

7. Perelson, A.S., Nelson, P.W.: Mathematical analysis of HIV-1 dynamics in vivo. SIAM Rev. 41(1), 3-44 (1999)

8. Callaway, D.S., Perelson, A.S.: HIV-1 infection and low steady state viral loads. Bull. Math. Biol. 64(1), 29-64 (2002)

9. Elaiw, A.M., Azoz, S.A.: Global properties of a class of HIV infection models with BeddingtonDeAngelis functional response. Math. Methods Appl. Sci. 36(4), 383-394 (2013)

10. Elaiw, A.M., Alshaikh, M.A.: Stability of discrete-time HIV dynamics models with three categories of infected CD4 ${ }^{+}$T-cells. Adv. Differ. Equ. 2019, Article Number: 407 (2019)

11. Elaiw, A.M., AlShamrani, N.H.: Stability of a general CTL-mediated immunity HIV infection model with silent infected cell-to-cell spread. Adv. Differ. Equ. 2020, 355 (2020)

12. Elaiw, A.M., Elnahary, E.K., Raezah, A.A.: Effect of cellular reservoirs and delays on the global dynamics of HIV. Adv. Differ. Equ. 2018(1), Article Number: 85 (2018)

13. Liu, H., Zhang, J.-F.: Dynamics of two time delays differential equation model to HIV latent infection. Phys. A 514(C), 384-395 (2019)

14. Lairmore, M.D., Haines, R., Anupam, R.: Mechanisms of human T lymphotropic virus type 1 transmission and disease. Curr. Opin. Virol. 2(4), 474-481 (2012)

15. Lim, A.G., Maini, P.K.: HTLV-I infection: a dynamic struggle between viral persistence and host immunity. J. Theor. Biol. 352, 92-108 (2014)

16. Pan, X., Chen, Y., Shu, H.: Rich dynamics in a delayed HTLV-I infection model: stability switch, multiple stable cycles, and torus. J. Math. Anal. Appl. 479(2), 2214-2235 (2019)

17. Asquith, B., Bangham, C.R.M.: The dynamics of T-cell fratricide: application of a robust approach to mathematical modeling in immunology. J. Theor. Biol. 222(1), 53-69 (2003)

18. Tokudome, S., et al.: Incidence of adult $\mathrm{T}$ cell leukemia/lymphoma among human $\mathrm{T}$ lymphotropic virus type 1 carriers in Saga, Japan. Cancer Res. 49(1), 226-228 (1989)

19. Stilianakis, N.I., Seydel, J.: Modeling the T-cell dynamics and pathogenesis of HTLV-I infection. Bull. Math. Biol. 61(5), 935-947 (1999)

20. Vargas-De-Leon, C.: The complete classification for global dynamics of a model for the persistence of HTLV-1 infection. Appl. Math. Comput. 237(15), 489-493 (2014)

21. Wang, L., Li, M.Y., Kirschner, D.: Mathematical analysis of the global dynamics of a model for HTLV-I infection and ATL progression. Math. Biosci. 179(2), 207-217 (2002) 
22. Yamamoto, N., Okada, M., Koyanagi, Y., Kannagi, M., Hinuma, Y.: Transformation of human leukocytes by cocultivation with an adult T cell leukemia virus producer cell line. Science 217(4561), 737-739 (1982)

23. Bangham, C.R.M.: The immune response to HTLV-I. Curr. Opin. Immunol. 12(4), 397-402 (2000)

24. Li, M.Y., Lim, A.G.: Modelling the role of Tax expression in HTLV-1 persisence in vivo. Bull. Math. Biol. 73(12), 3008-3029 (2011)

25. Asquith, B., Bangham, C.R.M.: Quantifying HTLV-I dynamics. Immunol. Cell Biol. 85(4), 280-286 (2007)

26. Rosenberg, E.S., Altfeld, M., Poon, S.H., et al.: Immune control of HIV-1 following early treatment of acute infection. Nature 407(6803), 523-526 (2000)

27. Shu, H., Wang, L., Watmough, J.: Global stability of a nonlinear viral infection model with infinitely distributed intracellular delays and CTL immune responses. SIAM J. Appl. Math. 73(3), 1280-1302 (2013)

28. Guo, T., Qiu, Z.: The effects of CTL immune response on HIV infection model with potent therapy, latently infected cells and cell-to-cell viral transmission. Math. Biosci. Eng. 16(6), 6822-6841 (2019)

29. Elaiw, A.M., AlShamrani, N.H.: Global stability of a delayed adaptive immunity viral infection with two routes of infection and multi-stages of infected cells. Commun. Nonlinear Sci. Numer. Simul. 86, Article ID 105259 (2020)

30. Bangham, C.R.M.: CTL quality and the control of human retroviral infections. Eur. J. Immunol. 39(7), 1700-1712 (2009)

31. Gomez-Acevedo, H., Li, M.Y., Jacobson, S.: Multi-stability in a model for CTL response to HTLV-I infection and its consequences in HAM/TSP development, and prevention. Bull. Math. Biol. 72(3), 681-696 (2010)

32. Li, M.Y., Shu, H.: Multiple stable periodic oscillations in a mathematical model of CTL response to HTLV-I infection. Bull. Math. Biol. 73(8), 1774-1793 (2011)

33. Wang, L., Liu, Z., Li, Y., Xu, D.: Complete dynamical analysis for a nonlinear HTLV-I infection model with distributed delay. CTL response and immune impairment. Discr. Contin. Dyn. Syst. 25(3), 917-933 (2020)

34. Wang, Y., Liu, J., Heffernan, J.M.: Viral dynamics of an HTLV-I infection model with intracellular delay and CTL immune response delay. J. Math. Anal. Appl. 459(1), 506-527 (2018)

35. Li, F., Ma, W.: Dynamics analysis of an HTLV-1 infection model with mitotic division of actively infected cells and delayed CTL immune response. Math. Methods Appl. Sci. 41(8), 3000-3017 (2018)

36. Li, S., Zhou, Y.: Backward bifurcation of an HTLV-I model with immune response. Discr. Contin. Dyn. Syst. Ser. B 21(3), 863-881 (2016)

37. Wang, W., Ma, W.: Global dynamics of a reaction and diffusion model for an HTLV-I infection with mitotic division of actively infected cells. J. Appl. Anal. Comput. 7(3), 899-930 (2017)

38. Pilotti, E., Bianchi, M.V., De Maria, A., Bozzano, F., Romanelli, M.G., Bertazzoni, U., Casoli, C.: HTLV-1/-2 and HIV-1 co-infections: retroviral interference on host immune status. Front. Microbiol. 4, Article ID 372 (2013)

39. Beilke, M.A., Theall, K.P., O’Brien, M., Clayton, J.L., Benjamin, S.M., Winsor, E.L., Kissinger, P.J.: Clinical outcomes and disease progression among patients coinfected with HIV and human T lymphotropic virus types 1 and 2. Clin. Infect. Dis. 39(2), 256-263 (2004)

40. Isache, C., Sands, M., Guzman, N., Figueroa, D.: HTLV-1 and HIV-1 co-infection: a case report and review of the literature. IDCases 4, 53-55 (2016)

41. Tulius Silva, M., de Melo Espíndola, O., Bezerra Leite, A.C., Araújo, A.: Neurological aspects of HIV/human T lymphotropic virus coinfection. AIDS Rev. 11(2), 71-78 (2009)

42. Rockwood, N., Cook, L., Kagdi, H., Basnayake, S., Bangham, C.R.M., Pozniak, A.L., Taylor, G.P.: Immune compromise in HIV-1/HTLV-1 coinfection with paradoxical resolution of CD4 lymphocytosis during antiretroviral therapy: A case report. Medicine 94(50), e2275 (2015)

43. Brites, C., Sampalo, J., Oliveira, A.: HIV/human T-cell lymphotropic virus coinfection revisited: impact on AIDS progression. AIDS Rev. 11(1), 8-16 (2009)

44. Ticona, E., Huaman, M.A., Yanque, O., Zunt, J.R.: HIV and HTLV-1 coinfection: the need to initiate antiretroviral therapy. J. Int. Assoc. Providers AIDS Care 12(6), 373-374 (2013)

45. Geddes, V.E.V., José, D.P., Leal, F.E., Nixond, D.F., Tanuri, A., Aguiar, R.S.: HTLV-1 Tax activates HIV-1 transcription in latency models. Virology 504, 45-51 (2017)

46. Bellomo, N., Painter, K.J., Tao, Y., Winkler, M.: Occurrence vs. absence of taxis-driven instabilities in a May-Nowak model for virus infection. SIAM J. Appl. Math. 79(5), 1990-2010 (2019) 
47. Elaiw, A.M., AlAgha, A.D.: Global analysis of a reaction-diffusion within-host malaria infection model with adaptive immune response. Mathematics 8, 563 (2020)

48. Nelson, P.W., Murray, J.D., Perelson, A.S.: A model of HIV-1 pathogenesis that includes an intracellular delay. Math. Biosci. 163(2), 201-215 (2000)

49. Elaiw, A.M., Raezah, A.A.: Stability of general virus dynamics models with both cellular and viral infections and delays. Math. Methods Appl. Sci. 40(16), 5863-5880 (2017)

50. Elaiw, A.M., Almuallem, N.A.: Global dynamics of delay-distributed HIV infection models with differential drug efficacy in cocirculating target cells. Math. Methods Appl. Sci. 39(1), 4-31 (2016)

51. Elaiw, A.M., Alshehaiween, S.F.: Global stability of delay-distributed viral infection model with two modes of viral transmission and B-cell impairment. Math. Methods Appl. Sci. 43(11), 6677-6701 (2020)

52. Culshaw, R.V., Ruan, S.: A delay-differential equation model of HIV infection of CD4+ T-cells. Math. Biosci. 165(1), 27-39 (2000)

53. Gibelli, L., Elaiw, A., Alghamdi, M.A., Althiabi, A.M.: Heterogeneous population dynamics of active particles: progression, mutations, and selection dynamics. Math. Methods Appl. Sci. 27(4), 617-640 (2017)

54. Barbashin, E.A.: Introduction to the Theory of Stability. Wolters-Noordhoff, Groningen (1970)

55. LaSalle, J.P.: The Stability of Dynamical Systems. SIAM, Philadelphia (1976)

56. Lyapunov, A.M.: The General Problem of the Stability of Motion. Taylor \& Francis Ltd, London (1992)

57. Perelson, A.S., Kirschner, D.E., de boer, R.: Dynamics of HIV infection of CD4+ T cells. Math. Biosci. 114, 81-125 (1993)

58. Mohri, H., Bonhoeffer, S., Monard, S., Perelson, A.S., Ho, D.D.: Rapid turnover of T lymphocytes in SIV-infected rhesus macaques. Science 279, 1223-1227 (1998)

59. Wodarz, D.: Mathematical models of immune effector responses to viral infections: virus control versus the development of pathology. J. Comput. Appl. Math. 184(1), 301-319 (2005)

60. Sahani, S.K., Yashi: Effects of eclipse phase and delay on the dynamics of HIV infection. J. Biol. Syst. 26(3), 421-454 (2018)

61. Vandormael, A., Rego, F., Danaviah, S., Carlos Junior Alcantara, L., Boulware, D.R., de Oliveira, T.: CD4+ T-cell count may not be a useful strategy to monitor antiretroviral therapy response in HTLV1/HIV co-infected patients. Curr. HIV Res. 15(3), 225-231 (2017)

62. Barski, M.S., Minnell, J.J., Maertens, G.N.: Inhibition of HTLV-1 infection by HIV-1 first- and second-generation integrase strand transfer inhibitors. Front. Microbiol. 10, Article ID: 1877 (2019)

Publisher's Note Springer Nature remains neutral with regard to jurisdictional claims in published maps and institutional affiliations.

\section{Authors and Affiliations}

\section{A. M. Elaiw ${ }^{1,2}$ (D) N. H. AlShamrani ${ }^{1,3}$}

A. M. Elaiw

a_m_elaiw@yahoo.com

N. H. AlShamrani

nhalshamrani@uj.edu.sa

1 Department of Mathematics, Faculty of Science, King Abdulaziz University, P.O. Box 80203, Jeddah 21589, Saudi Arabia

2 Department of Mathematics, Faculty of Science, Al-Azhar University, Assiut Branch, Assiut, Egypt

3 Department of Mathematics, Faculty of Science, University of Jeddah, P.O. Box 80327, Jeddah 21589, Saudi Arabia 